Portland State University

PDXScholar

\title{
Nuclear Magnetic Resonance Investigation of the Interaction of Heme Binding Proteins with SnIVprotoporphyrin IX and Heme: Structure and Conformational Changes of Myoglobin and Hemopexin
}

Ruba Saba Deeb

Portland State University

Follow this and additional works at: https://pdxscholar.library.pdx.edu/open_access_etds Let us know how access to this document benefits you.

\section{Recommended Citation}

Deeb, Ruba Saba, "Nuclear Magnetic Resonance Investigation of the Interaction of Heme Binding Proteins with SnIVprotoporphyrin IX and Heme: Structure and Conformational Changes of Myoglobin and Hemopexin" (1993). Dissertations and Theses. Paper 1368.

https://doi.org/10.15760/etd.1367

This Dissertation is brought to you for free and open access. It has been accepted for inclusion in Dissertations and Theses by an authorized administrator of PDXScholar. Please contact us if we can make this document more accessible: pdxscholar@pdx.edu. 
NUCLEAR MAGNETIC RESONANCE INVESTIGATION OF THE

INTERACTION OF HEME BINDING PROTEINS WITH

SNIVPROTOPORPHYRIN IX AND HEME: STRUCTURE AND

CONFORMATIONAL CHANGES OF MYOGLOBIN AND HEMOPEXIN

by

RUBA SABA DEEB

\begin{abstract}
A dissertation submitted in partial fulfillment of the requirements for the degree of

DOCTOR OF PHILOSOPHY

in

ENVIRONMENTAL SCIENCES AND RESOURCES:

CHEMISTRY
\end{abstract}

Portland State University

1993 


\section{TO THE OFFICE OF GRADUATE STUDIES}

The members of the Committee approve the dissertation of Ruba Saba Deeb presented April 22, 1993.

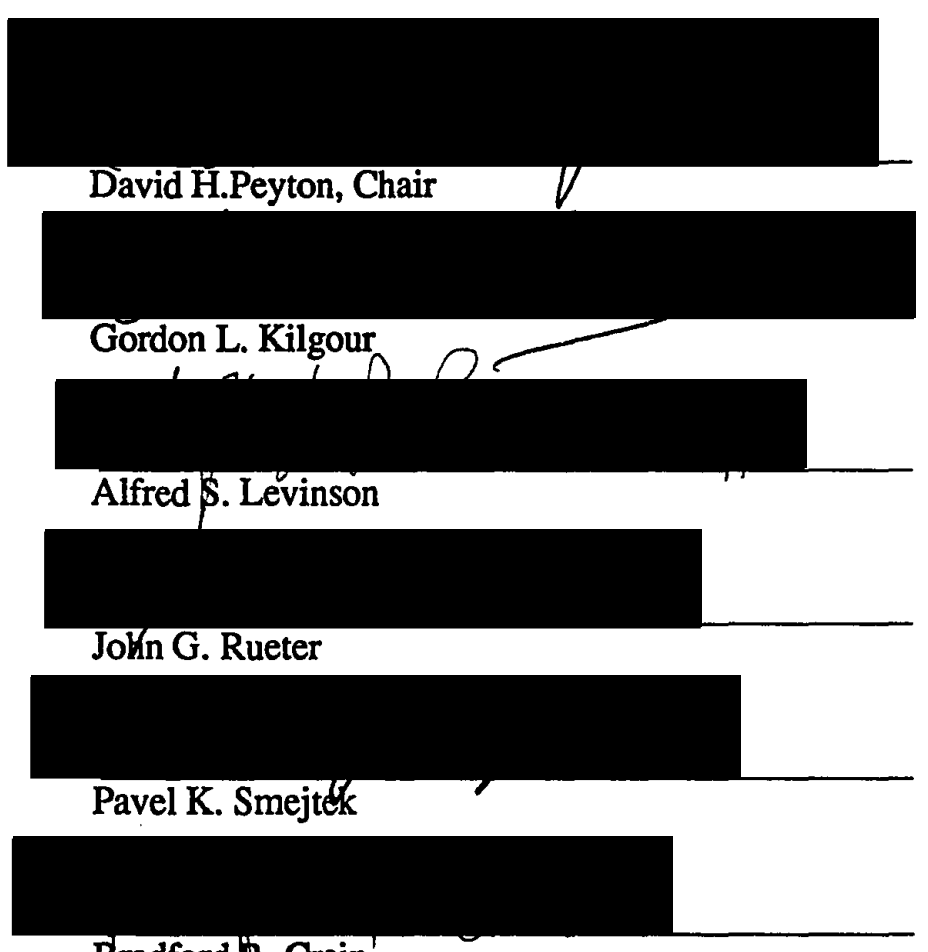

Bradford R. Crain

\section{APPRQVED:}

Robert O. Tinnin, Acting Dean, College of Liberal Arts and Sciences

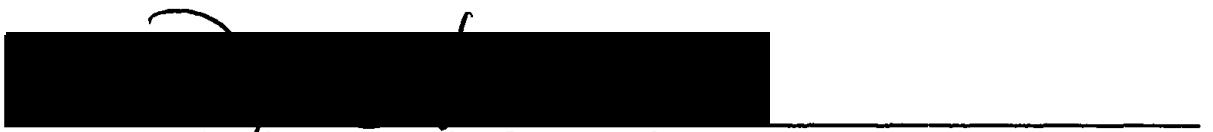

Roy W. Koch, Yice Provost for Graduate Studies and Research 
AN ABSTRACT OF THE DISSERTATION OF Ruba Saba Deeb for the Doctor of Philosophy in Environmental Sciences and Resources: Chemistry presented April 22, 1993.

Title: Nuclear Magnetic Resonance Investigation of the Interaction of Heme Binding Proteins with $\mathrm{Sn}^{\mathrm{I}}$ Protoporphyrin $\mathrm{XX}$ and Heme: Structure and Conformational Changes of Myoglobin and Hemopexin.

\section{APPROVED BY THE MEMBERS OF THE DISSERTATION COMMITTEE:}

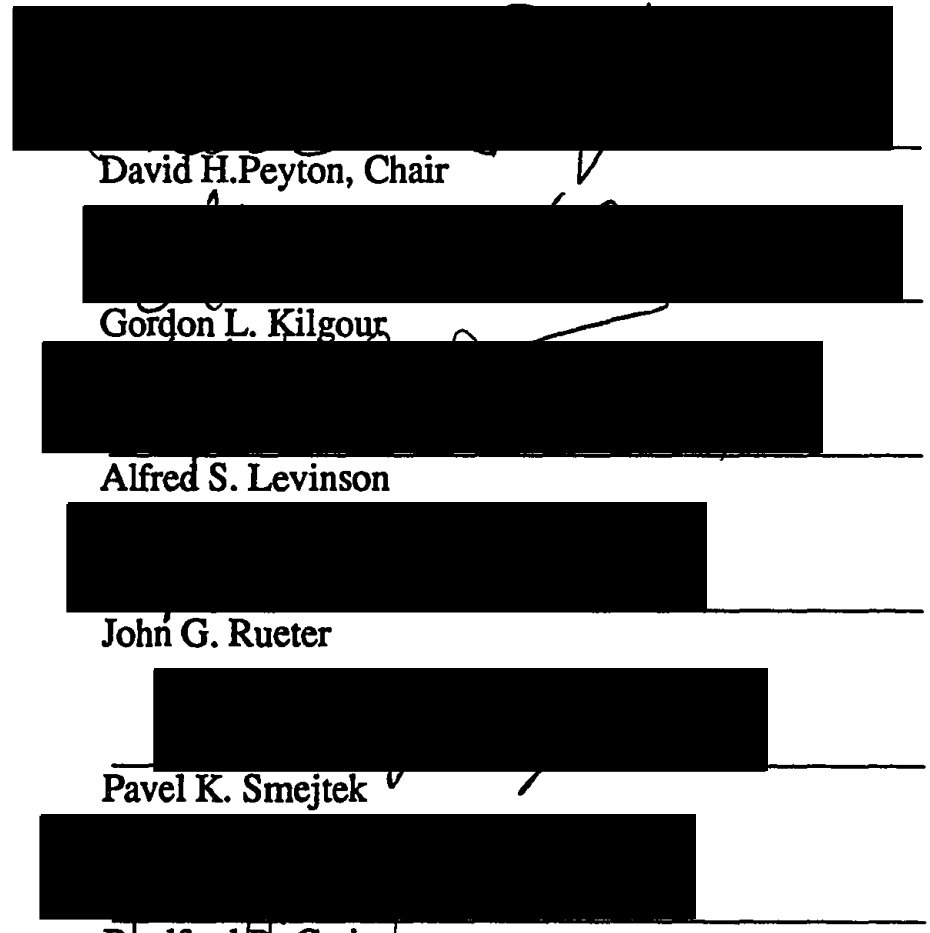

Btadford B. Crain

Tin protoporphyrin $\mathrm{XX}(\mathrm{SnPP})$ is currently under investigation for the treatment of hyperbilirubinemia. The study of the complex between SnPP and equine myoglobin 
(EqMb) by ${ }^{1} \mathrm{H}$ and ${ }^{119} \mathrm{Sn}$ nuclear magnetic resonance spectroscopy (NMR) can be viewed as a general model for SnPP interaction with hemoproteins.

The complex formed from the equilibrium mixture of SnPP and EqMb, SnPP•EqMb, was found to have essentially the same porphyrin-binding pocket as EqMbCO and SwMbCO, including the same porphyrin orientation in the major form of the two species. ${ }^{119}$ Sn NMR spectroscopy was used to demonstrate that the proximal His(93)F8-metal coordination is likely to be intact in SnPP.EqMb. Minor shifts in the side chain positions of some of the residues were observed, possibly reflecting the presence of water in the sixth coordination site. SnPP.EqMb appears to be stable; it persists at room temperature for weeks and exhibits very slow exchange rates $\left({ }^{2} \mathrm{H}\right.$ for $\left.{ }^{1} \mathrm{H}\right)$ for a large number of amide protons in the $\mathrm{pH}$ range 7-9.

Events during the reconstitution of apomyoglobin (apoMb) with SnPP were probed. Thus interactions between tin(IV)protoporphyrin IX (SnPP) and equine apoMb, and between tin(IV)protoporphyrin IX dimers (SnPP) 2 and apoMb were observed by ${ }^{1} \mathbf{H}$ NMR and optical spectroscopic techniques. The products and intermediates observed in this situation were related to the equilibrium structure of SnPP.EqMb. Reactions of apoEqMb with $\mathrm{SnPP}$ and $(\mathrm{SnPP})_{2}$ produce different intermediates, although the final product, SnPP.EqMb, is the same for each. An intermediate observed for the reaction of $\mathrm{SnPP}$ with apoEqMb at $\mathrm{pH} 10$ is in exchange with free SnPP, with the observed rate constant $K_{\text {off }} \sim 1 \mathrm{~s}^{-1}$; meso-proton resonances were assigned for this intermediate by correlation to SnPP resonances via chemical exchange. The intermediate observed for the reaction of $(\mathrm{SnPP})_{2}$ with apoEqMb at neutral $\mathrm{pH}$ produces another species which may be the alternate porphyrin-insertion isomer arising from a $180^{\circ}$ rotation about the $\alpha, \gamma$ meso axis of the porphyrin. Although optical absorbance spectroscopy of the Soret region shows evidence for the reaction of $\mathrm{SnPP}$ and $(\mathrm{SnPP})_{2}$ with apoMb, only in combination with ${ }^{1} \mathrm{H}$ NMR are the various processes assigned. 
The study of the complex SnPP.EqMb facilitated the investigation of the more complex heme binding protein, hemopexin ( $\mathrm{Hx}$ ). Proton NMR spectroscopy is reported for the first time for the hemin complex of hemopexin, a serum protein that binds heme exceptionally tightly. Hx from cow, rat, rabbit, and human was isolated, and data for the protein were reported. Heme-bound $\mathrm{Hx}$ has spectral characteristics for being low-spin, paramagnetic. Deuterium isotope labels reveal the positions for the heme 1-, 3-, and 8methyls; the 5-methyl lies in the -5 to $12 \mathrm{ppm}$ region. Furthermore, two-dimensional nuclear Overhauser effect spectroscopy was used to locate other heme periphery protons, including those from the 2-vinyl and the 7-propionate. Upfield resonances are identified that are very strongly relaxed, and so are assigned to protons on the axial ligands. The information reported here contributes to the understanding of $\mathrm{Hx}$ as an antioxidant at the cellular level. 


\section{DEDICATION}

For your unending faith in me, this dissertation is dedicated to my father, Saba, my mother, May, my uncle, Elias Kari, and my cousin Ghassan Dib. 


\section{ACKNOWLEDGEMENTS}

Dr. Peyton: I couldn't have hoped for a better scientific father. Thank you; Dr. Kilgour: Thank you for all the encouragement and wonderful advice; Members of my dissertation committee: Dr. Gordon Kilgour, Dr. Alfred Levinson, Dr. John Rueter, and Dr. Pavel Smejtek, thank you for your time; Dr. Ursula Muller-Eberhard: Thank you for your support and faith in my work; Carol Dib: You are a universal role model, and have my admiration and love. Thank you for believing in me; Sheri Opp: Some of the best parts of the last four years are because of you. You are the best friend I've had and will have. Thank you; Imad Deeb: Your confidence in me meant so much. You have been a great brother; Ruben Torres: Thank you for being like a brother to me; Verna Burden: You are an angel, your dolphin stories will always remain with me; Elisar, Sidney and Dietmar: You made Dr. Peyton's lab a place I will never forget. 
TABLE OF CONTENTS

PAGE

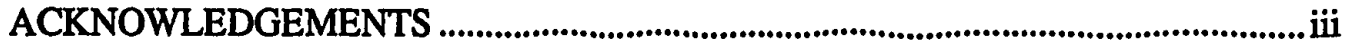

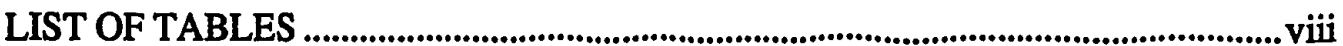

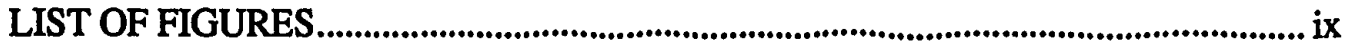

DEFINITIONS AND ABBREVIATIONS ...................................................................xii

CHAPTER

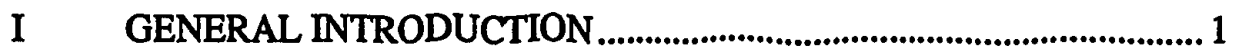

Significance of the Research .............................................................. 2

Damage by Lead at the Cellular Level............................................... 3

Damage by Carbon Tetrachloride at the Cellular Level ..................... 6

Hemopexin as an Antioxidant............................................................

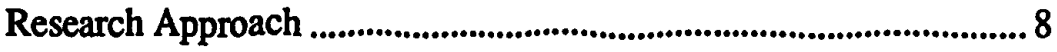

II OPTICAL SPECTROSCOPY AND NUCLEAR MAGNETIC

RESONANCE SPECTROSCOPY INSTRUMENTATION .............9

III PREPARATION, ISOLATION, AND PURIFICATION

TECHNIQUES FOR APOMYOGLOBIN FROM HORSE

HEART, AND HEMOPEXIN FROM VARIOUS SPECIES

(COW, RAT, HUMAN, RABBIT) ............................................. 13

Preparation of Equine Apomyoglobin................................................ 13

Preparation of Heme-Agarose Column ............................................. 14 
Isolation and Purification of Hemopexin ( $\mathrm{Hx}$ ) from Bovine

Plasma with Heme-Agarose.......................................................... 14

Isolation and Purification of Hemopexin from Serum by Ion-

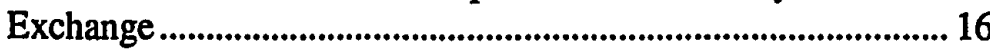

Reconstitution of Hemopexin with Protohemin IX........................... 17

IV CHARACTERIZATION AND PROPERTIES OF SNPP AND

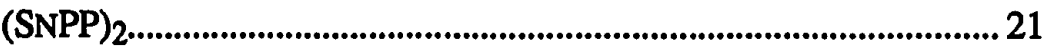

Materials and Methods ....................................................................... 21

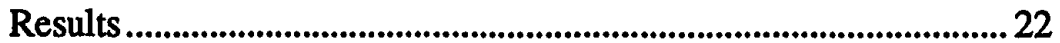

SnPP and (SnPP) ${ }_{2}{ }^{1} \mathrm{H}$ NMR Resonance Assignments

${ }_{119} \mathrm{Sn}$ NMR of SnPP and (SnPP) 2

${ }^{13}$ C NMR Assignments of SnPP

FTIR for SnPP and (SnPP) 2

Optical Spectroscopic Observation of the Kinetics of SnPP and $(\mathrm{SnPP})_{2}$

Discussion

V ${ }^{1} \mathrm{H}$ AND ${ }^{119}$ SN MAGNETIC RESONANCE STUDY OF THE

PORPHYRIN-BINDING POCKET OF THE EQUILIBRIUM

STRUCTURE OF TIN (IV) PROTOPORPHYRIN IX

COMPLEX OF EQUINE MYOGLOBIN

Materials and Methods............................................................................... 34

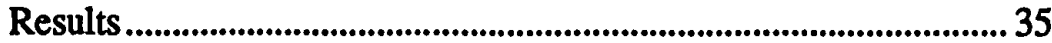

The ${ }^{1} \mathrm{H}$ NMR Spectrum of SnPP•EqMb

Assignment of Porphyrin resonances

Phe(43)CD1 (Distal)

Phe(33)B14 (Distal)

Phe(46)CD4 (Distal)

Val(68)E11 (Distal)

Leu(29)B10 (Distal)

His(64)E7 (Distal)

Ile(99)FG5 (Proximal)

His(97)FG3 (Proximal) 
The Proximal His-Fe Bond: ${ }^{119}$ Sn NMR

The Distal Fe-OH(2): ${ }^{1} \mathrm{H}$ NMR pH-Titration

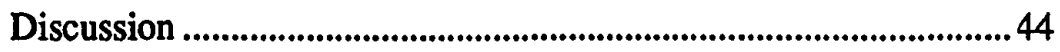

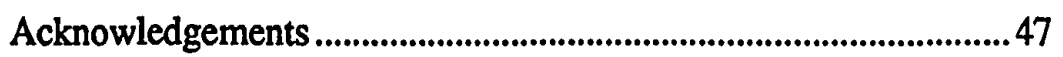

VI PROTON NMR STUDY OF THE INTERACTION OF TIN (IV) PROTOPORPHYRIN IX MONOMERS AND DIMERS

WITH APOMYOGLOBIN ...........................................................56

Materials and Methods ..............................................................57

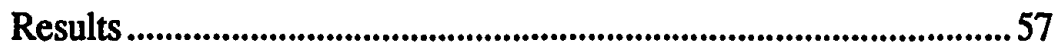

${ }^{1} \mathrm{H}$ NMR Observation of an Intermediate in the Process:

$$
\mathrm{SnPP}+\mathrm{ApoEqMb} \rightarrow \mathrm{SnPP} \cdot \mathrm{EqMb}
$$

Optical Spectroscopic Observation of an Intermediate in the

Process: SnPP + ApoEqMb $\rightarrow$ SnPP•EqMb

${ }^{1} \mathrm{H}$ NMR Observation of an Intermediate in the Process:

$(\mathrm{SnPP})_{2}+2 \mathrm{ApoEqMb} \rightarrow 2 \mathrm{SnPP} \cdot \mathrm{EqMb}$

Optical Spectroscopic Observation of the Kinetics of the

Process: (SnPP) $2+2 \mathrm{ApoEqMb} \rightarrow 2 \mathrm{SnPP} \cdot \mathrm{EqMb}$

Discussion

VII CHARACTERIZATION OF HEMOPEXIN BY SDSPOLYACRYLAMIDE, GEL ELECTROPHORESIS AND OPTICAL SPECTROSCOPY. . .78

Materials and Methods ...............................................................79

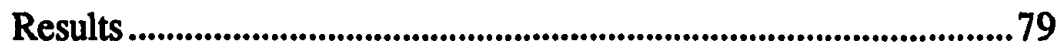

Isolation of Rat ApoHx by Ion Exchange and Gel

Exclusion Chromatography

Initial Modification of ApoHx Isolation

Heme•Hx Isolation

Discussion 
VIII PROTON NMR STUDY OF THE HEME COMPLEX OF

HEMOPEXIN

Materials and Methods .............................................................. 90

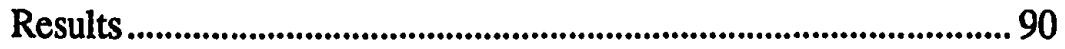
Species Comparison
Cyanide Complex of Rabbit Heme•Hx
Competition Studies
Peak Assignments by Isotope-Labeling Experiments
NOESY of Heme $\cdot \mathrm{Hx}_{\text {rabbit }}$

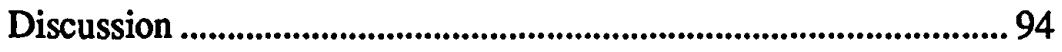

Acknowledgements ...................................................................96

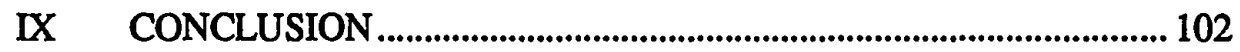

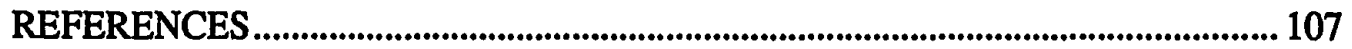




\section{LIST OF TABLES}

TABLE

PAGE

I Various methods used for isolating $\mathrm{Hx}$ from plasma .........................20

II $\quad{ }^{1} \mathrm{H}$ and ${ }^{13} \mathrm{C}$ NMR chemical shifts for SnPP and

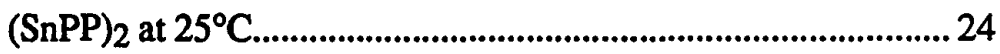

III 1 $_{\mathrm{H}}$ NMR chemical shifts for various myoglobins in ppm.................... 45

IV NMR chemical shifts for SnPP.EqMb* and

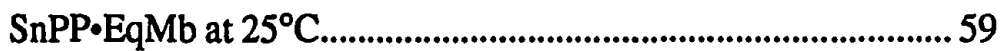

V NMR parameters for Rabbit Heme•Hx ...........................................95 95 


\section{LIST OF FIGURES}

FIGURE

PAGE

1. Schematic diagram representing cellular damage by

bilirubin and heme.......................................................................... 3

2. Schematic representation of Conformational change occurring in $\mathrm{Hx}$ upon heme binding......................................... 8

3. Schematic diagram for the preparation of Heme-Agarose column....... 15

4. Schematic diagram for the isolation of hemopexin by the Heme-Agarose method................................................................ 17

5. Schematic diagram for the isolation and purification of hemopexin by a modified technique ..................................... 19

6. PPIX skeleton showing intersubstituent NOEs (arcs) observed in ROESY spectra of SnPP and (SnPP) 2................................... 27

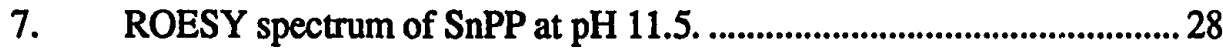

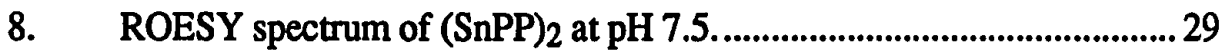

9. ${ }^{119} \mathrm{Sn}$ NMR of a mixture of $\mathrm{SnPP}$ and $(\mathrm{SnPP})_{2}$ illustrating a chemical shift change of $+13.1 \mathrm{ppm}$ between SnPP and (SnPP) 2 ............... 30

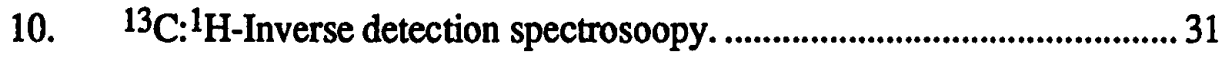

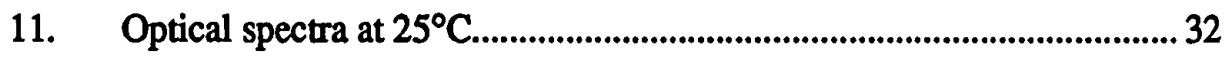

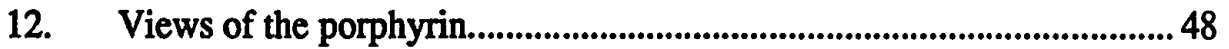

13. One-dimensional NMR spectroscopy portions showing upfield and downfield regions 
14. DQF-COSY/NOESY spectroscopy of SnPP•EqMb at $\mathrm{pH} 9.0 \ldots \ldots . . . . . .50$

15. Two-dimensional ${ }^{1} \mathrm{H}$ NMR spectra from the aromatic region of SnPP·EqMb at $\mathrm{pH} 9.0$.

16. Two-quantum ${ }^{1} \mathrm{H}$ NMR spectroscopy showing the Phe aromatic spin system connectivities.

17. Two-dimensional ${ }^{1} \mathrm{H}$ NMR spectra from the aliphatic region of SnPP.EqMb at $\mathrm{pH} 9.0$

18. ${ }^{119} \mathrm{Sn}$ spectroscopy of SnPP•EqMb at pH 9.0.................................5 54

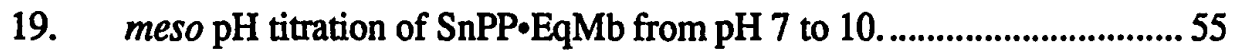

20. Saturation-transfer for SnPP•EqMb ${ }^{*}$ and $\mathrm{SnPP} \cdot \mathrm{EqM} b$ in the presence of excess SnPP monomer at $\mathrm{pH} 10.2$ and $25^{\circ} \mathrm{C}$

21. Saturation-transfer difference spectra for SnPP.EqMb* in the presence of excess SnPP monomer at $\mathrm{pH} 10.2$, at $10^{\circ}$ and $25^{\circ} \mathrm{C}$

22. NOE difference spectra resulting from irradiating the upfield resolved $\mathrm{Val}(68) \mathrm{E} 11 \mathrm{\gamma CH}_{3}$ at $\mathrm{pH} 10.2$ and $25^{\circ} \mathrm{C}$.

23. Optical spectra of the Soret region which reflect the conversion of $\mathrm{SnPP} \cdot \mathrm{EqMb} *$ to SnPP•EqMb at $\mathrm{pH} 10.0$ and $25^{\circ} \mathrm{C}$ .70

24. ${ }^{1} \mathrm{H}$ NMR observation of the incorporation of (SnPP) ${ }_{2}$ into apoEqMb in the downfield region at $\mathrm{pH} 7.5$ and $25^{\circ} \mathrm{C}$.

25. Optical spectra of the Soret region which reflect the conversion of $(\mathrm{SnPP})_{2} \cdot \mathrm{EqMb}$ to $\mathrm{SnPP} \cdot \mathrm{EqMb}$ at $\mathrm{pH} 7.1$

26. A graph showing the dissociation rate of $(\mathrm{SnPP})_{2}$ in the presence of apoMb $\left(1.7 \times 10^{-2} \mathrm{~min}^{-1}\right)$ and without apoMb $\left(8.8 \times 10^{-3} \mathrm{~min}^{-1}\right)$.

27. A schematic diagram illustrating the mechanism of dimer interaction with apoMb 
28. A schematic diagram illustrating thetwo species SnPP•EqMb* and SnPP•EqMb.

29. An illustration of the tight fit of the myoglobin pocket

(Evans and Brayer, 1988). 76

30. Optical spectrum of SnPP•EqMb showing the Q-band splitting ...........77

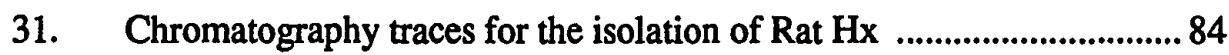

32. Optical spectra of Rat oxyhemoglobin (right) and hemopexin (left) .85

33. SDS-PAGE gels 86

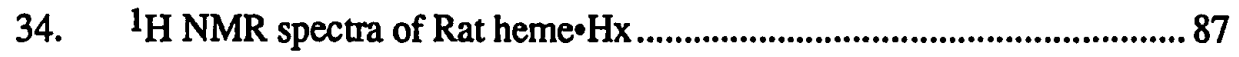

35. Chromatography traces for the isolation of Rabbit $\mathrm{Hx}$.........................88

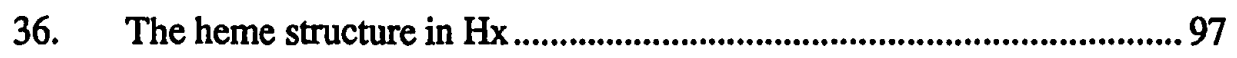

37. Species comparison for the hyperfine-shifted portions of the ${ }^{1} \mathrm{H}$ NMR spectrum of heme $\mathrm{Hx}$.....................................98

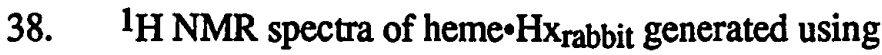
isotopically-labeled hemin.

39. Two-dimensional $20 \mathrm{~ms}$ mixing time NOESY and reference spectrum of heme-Hx $x_{\text {rabbit }}$ 100

40. ${ }^{1}$ H NMR spectra of heme $\cdot H_{x_{\text {rabbit }}}$ illustrating its temperature dependence. 


\section{DEFINITIONS AND ABBREVIATIONS}

ApoMb: myoglobin without the heme group; DQF-COSY: double-quantum filtered correlation spectroscopy. Two-dimensional correlation giving through bond interactions. This technique identifies three-bond interactions; Holoprotein: a protein with its prosthetic group; Multiple-Quantum Spectroscopy: A technique that includes stepwise editing of ${ }^{1} \mathrm{H}$ NMR spectra into subspectra devoid of spin systems with two, three, four, and five protons by use two, three, four, five and six quantum spectroscopy; NOESY: nuclear Overhauser effect spectroscopy. Two-dimensional correlation giving through space interactions. The NOE is related both to how far in space the nuclei are separated, and to the velocity of the internuclear vector; Null Method: a method for measuring the relaxation time $T_{1}$ by the inversion-recovery method because the first step inverts the magnetization. $T_{1}=\tau_{\text {null }} / \mathrm{ln} 2$ such that $\tau_{\text {null }}$ is the time where the signal will be zero; ROESY: rotating-frame nuclear Overhauser effect spectroscopy. The major advantage of doing ROESY is that all NOEs (sometimes called ROEs) are positive, regardless of molecular tumbling rate. Therefore, the intermediate-sized molecules which give small measurable but positive NOEs in the rotating frame; TOCSY: total correlation spectroscopy. Spin-locking experiments such that COSY cross peaks arise between all nuclei of a spin system; Waltz Sequence: a very efficient, low-power ${ }^{1} \mathrm{H}$-decoupling sequence.

$\mathrm{CD}$, circular dichroism; DesFeSwMb; EqMb, equine myoglobin; EqMbCN, cyano equine myoglobin; EqMbCO, carbon monoxy equine myoglobin; FTIR, Fourier transform infra red; Hb, hemoglobin; Heme, iron protoporphyrin IX; Hemin, iron(III)protoporphyrin IX; Hx, hemopexin; Mb, myoglobin; MbCO, carbonmonoxy 
myoglobin; mesoheme; MetHb, hemoglobin (Fe ${ }^{\mathrm{III}}$ ); $\mathrm{MetEqMbH}_{2} \mathrm{O}$; MetMb, myoglobin(FeIII); NMR, nuclear magnetic resonance; NOE, nuclear Overhauser effect; PPIX, protoporphyrin IX; SnPP, tin (IV) protoporphyrin IX; SwMb, sperm whale myoglobin; SwMbCO, carbon monoxy sperm whale myoglobin; TPPI, time proportional phase incrementation. 


\section{CHAPTER I}

\section{GENERAL INTRODUCTION}

The interactions of porphyrins with proteins are important from a number of perspectives. For example, about $70 \%$ of the iron in mammals is present as porphyrin complexes (hemes; Bertini and Luchinat, 1986) and, in this state, is responsible for processes ranging from oxygen or electron transport to the enzymatic processes of peroxidases and monoxygenases. The work presented in this dissertation involves studying metalloporphyrin-protein interactions for three reasons. First, free heme catalyzes unwanted oxidation reactions in the circulatory system. Second, the $\mathrm{Sn}$-for-Fe substituted porphyrin, $\operatorname{SnPP}\left(\mathrm{Sn}^{\mathrm{IV}}\right.$ protoporphyrin $\left.\mathrm{IX}\right)$ is a specific inhibitor of microsomal heme oxygenase (Drummond and Kappas, 1981), and so is being evaluated as a potential therapeutic agent against neonatal hyperbilirubinemia (this would be especially important in third world countries where controlled phototherapy is not always possible). Third, the Sn-for-Fe substitution allows the study of the role of metal-to-amino acid side chain coordination in protein folding and stability.

Myoglobin (Mb) and hemopexin ( $\mathrm{Hx}$ ) from various species were used as protein case studies for the interaction of SnPP and heme with heme binding proteins. Mb served as a well understood heme binding protein model. This model was used to study the behavior of SnPP upon binding. Hx was a far more difficult protein to isolate and study. This is due to its carbohydrate heterogeneity and high degree of glycosylation (Noiva et al., 1987). Hx is the tightest heme binding protein in the circulatory system, and current research is involved in studying its role in delivering the toxic heme to the liver. 


\section{SIGNIFICANCE OF THE RESEARCH}

Our environment now has numerous pollutants which give oxygen-derived radicals, for example paraquat, cigarette smoke, quinone anti-tumor drugs, alloxan, carbon tetrachloride, lead, and even some Spanish cooking oils (Halliwell and Gutteridge, 1986). These species are proposed to cause increased formation of superoxide radicals and hydrogen peroxide in vivo. Hydrogen peroxide and superoxide radicals can then interact with each other in the presence of metal ions to form a highly reactive species $(\cdot \mathrm{OH}$ radical) which can attack almost every molecule in living systems. The work presented in this dissertation interacts with this in that circulating heme-binding proteins, especially $\mathrm{Hx}$, are antioxidants that insure the absence of large quantities of low molecular-mass iron (such as unbound heme) from normal human plasma at physiological pH. Stress to cells from environmental pollution leads to an increased rate of protein damage including hemoglobin and myoglobin, resulting in increased levels of unbound heme (two examples are discussed below). This unbound heme would be involved in oxidative stress mechanisms by catalyzing lipid peroxidation and formation of the $\bullet \mathrm{OH}$ radical

(Figure 1), especially in individuals who are under treatment for porphyria (Sinclair et al., 1988).

$\mathrm{Hx}$ is an acute-phase reactant in all mammals, as evidenced by the observation that Hx levels at least quadruple within a few days of injection with turpentine or certain potential pollutants (Muller-Eberhard, 1988). This response points to Hx helping species to cope with environmental stress, in addition to its function in recycling iron. It should also be pointed out that when the heme is brought into the liver, it can either be inserted into another protein (such as has been shown for cytochrome P450) or it can be broken down. The iron would then be re-used. Either way, this leads to less demand for environmental iron. 


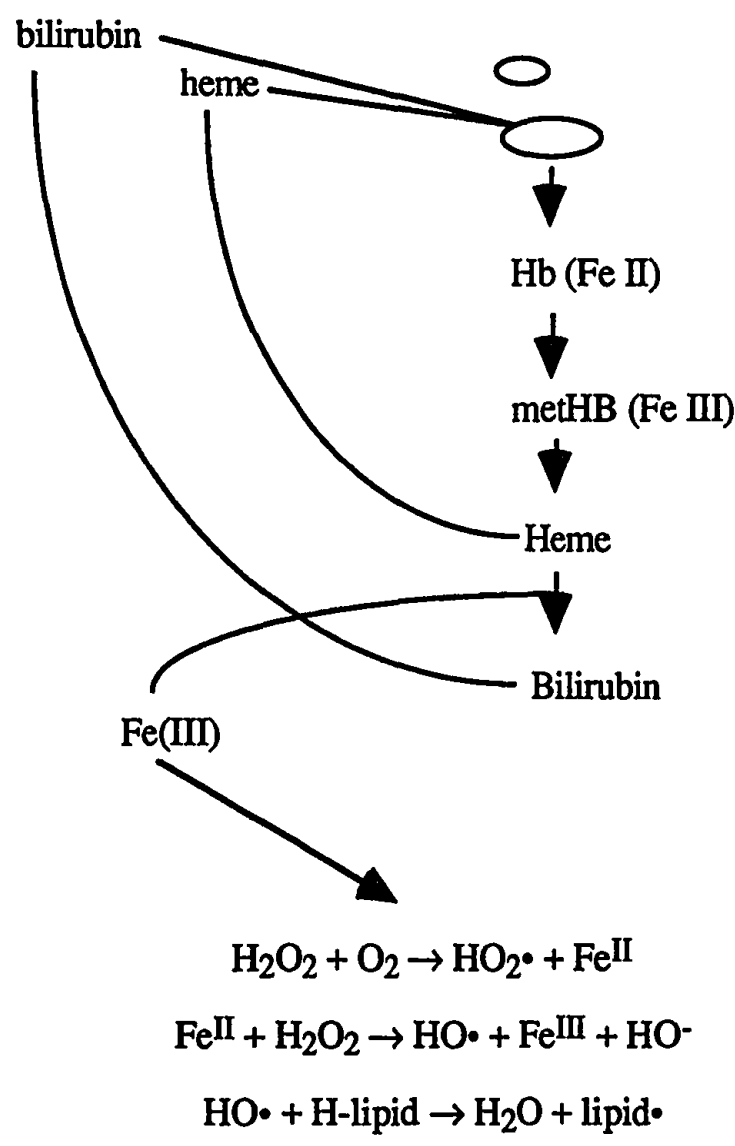

Eigure 1. Schematic diagram representing cellular damage by bilirubin and heme.

\section{DAMAGE BY LEAD AT THE CELLULAR LEVEL}

Lead, the most abundant toxic metal, is detectable in practically all phases of the inert environment and in all biologic systems. It is toxic to most species at high exposures, and has no demonstrated biologic need. The principle route of exposure is food; however, other sources produce excess exposure and toxic effects. Some of these sources include lead-based indoor paint in old dwellings, and lead in air from combustion of lead-containing auto fuels or industrial emissions (Mahaffey, 1985; Landrigan, 1989). 
The effect of lead relevant to this work is its disturbance of the heme biosynthesis pathway. This takes place at three points: inhibition of $\delta$-aminolevulinic acid dehydrase (ALA-D), depression of ferrochelatase (FERRO-C) activity, and raising of heme oxygenase activity (Bessis and Jensen, 1965). The series of equations presented below will explain the purpose of these enzymes as well as the results of their inhibition (Rawn, 1989). The key enzymes are underlined.

Heme Biosysnthesis

Succinyl CoA + Glycine + ALA-S $\rightarrow \delta$-aminolevulinic acid ( $\delta$-ALA)

$\delta$-ALA $+\underline{\text { ALA-D }} \rightarrow$ porphobillinogen (PBG)

PBG + PBG-Synthase $\rightarrow$ uroporphyrinogen (UROPOR)

UROPOR + UROPOR-Decarboxylase $\rightarrow$ coproporphyrinogen (COPRO)

COPRO + COPRO-Oxidase $\rightarrow$ protporphyrin IX (PPIX)

PPIX + FERRO-C $\rightarrow$ Iron (Fe ${ }^{\text {II }}$-PPIX (Heme)

Heme (II) + Globin $\rightarrow$ Hemoglobin $(\mathrm{Hb})$

Hemoglobin Degradation

$\mathrm{Hb}$ (II) $\rightarrow$ metHb (III) $\rightarrow$ Globin + Heme (III)

Heme (III) + Heme Oxygenase $\rightarrow$ Biliverdin

Biliverdin + Biliverdin Reductase $\rightarrow$ Bilirubin

Decreased synthesis of heme due to inhibition of ALA-D and FERRO-C activity in the cells results in marked increase of $\delta$-ALA levels in circulating blood and urinary excretion by virtue of feedback control. Furthermore, increased activity of heme oxygenase in the cells leads to increasing levels of bilirubin in the circulatory system. A combination of increasing heme degradation and a decrease in heme synthesis can cause anemia due to lack of sufficient heme production, which is needed for ongoing incorporation of heme into the globin. This however is not the only cause for concern. First, bilirubin is known to be a neurotoxin as well as an agent that promotes lysis of red 
blood cells by binding to the membrane cytoskeleton (Drummond and Kappas, 1984;

Kirschner-Zilber et al., 1982). The destruction of red blood cells facilitates the release of heme from the globin, and therefore aggravates anemic conditions induced by lead.

Furthermore, red cell lysis promotes the circulation of free heme. Second, excess $\delta$-ALA is proposed to undergo autoxidation into reactive oxygen species $\left(\mathrm{H}_{2} \mathrm{O}_{2}\right.$ and $\left.\mathrm{O}_{2}\right)$

(Hermes-Lima et al., 1991). The presence of free heme and reactive oxygen species in the circulatory system facilitate radical reactions with biologically important macromolecules such as membrane lipids, by hydrogen abstraction. This is demonstrated by the radical reactions presented below (Tappel, 1955; Halliwell and Gutteridge, 1986).

Lipid degradation by $\cdot \mathrm{OH}$

$$
\begin{aligned}
& \mathrm{H}_{2} \mathrm{O}_{2}+\mathrm{O}_{2}+\mathrm{Fe}^{+2} \rightarrow \cdot \mathrm{OH} \text { (Haber-Weiss reaction) } \\
& \cdot \mathrm{OH}+\text { lipid-H } \rightarrow \text { lipid } \bullet+\mathrm{H}_{2} \mathrm{O} \\
& \text { lipid }+\mathrm{O}_{2} \rightarrow \text { lipid- } \mathrm{O}_{2} \text { (peroxy radical) } \\
& \text { lipid- } \mathrm{O}_{2}^{\bullet}+\text { lipid-H } \rightarrow \text { lipid } \bullet+\text { lipid- } \mathrm{O}_{2} \mathrm{H} \text { (lipid peroxide) } \\
& \text { and so on. }
\end{aligned}
$$

\section{Lipid degradation by heme}

$\mathrm{R}-\mathrm{CH}=\mathrm{CH}-\mathrm{CH}=\mathrm{CH}-\mathrm{CHOOHR}+\mathrm{Heme}$ (hydroxy ligand) $\rightarrow \mathrm{H}_{2} \mathrm{O}+$ Adduct

The adduct is (Iron porphyrin)-0-0-(peroxide) which dissociates into:

Heme radical (hemeO-) and $\mathrm{R}-\mathrm{CH}=\mathrm{CH}-\mathrm{CH}=\mathrm{CH}-\mathrm{CHO} \cdot-\mathrm{R}$ and so on.

In a situation of lead poisoning with symptoms of increased hemolysis, Hx plays the role of an extracellular antioxidant. $\mathrm{Hx}$ binds free heme in the circulatory system and delivers it to intracellular sites in the liver (lysosome vesicles). In the lysosome vesicles, heme can be catabolized and the iron extracted safely, while the unbound Hx returns to the extracellular transport system (Smith and Hunt, 1990). Due to the strong affinity of heme for $\mathrm{Hx}\left(\mathrm{Kd} \sim 10^{-13}\right)$, studies have shown that $\mathrm{Hx}$ is capable of removing bound 
heme from membranes of red blood cells if $\mathrm{Hx}$ is present in sufficient quantity (Solar et al., 1989). This presents $\mathrm{Hx}$ as a potential drug to be further investigated.

$\mathrm{SnPP}$ is also a potential drug being investigated for the treatment of jaundice in newborn infants, through its inhibition of heme-oxygenase. This can certainly apply to cases of increased bilirubin synthesis due to lead toxicity.

\section{DAMAGE BY CARBON TETRACHLORIDE AT THE CELLULAR LEVEL}

Fumigants range from acrylonitrile to carbon tetrachloride $\left(\mathrm{CCl}_{4}\right)$. They are used to kill insects, nematodes, weed seeds and fungi in soil, silo-stored cereal grains, fruit, vegetables, cloths, and so forth, generally with the treatment being carried out in enclosed spaces because of the volatility of most of the products. These chemicals are nonselective and extremely toxic at elevated concentrations (Cremlyn, 1978; Hayes, 1982; Morgan, 1982).

The reductive dehalogenation of $\mathrm{CCl}_{4}$ by cytochrome $\mathrm{P} 450$ enzymes produces free radical intermediates owing to the uptake of the reducing equivalent (electron). The free radical can then interact with important biological macromolecules such as phospholipids that make up membranes, thereby generating a cascading and destructive radical reaction set seen in the equations below (Morgan, 1982).

$$
\begin{aligned}
& \mathrm{CCl}_{4}+\mathrm{Cyt} \mathrm{P450(e^{- } ) \rightarrow \cdot C C l 3}+\mathrm{Cl}^{-} \\
& \cdot \mathrm{CCl} 3+\text { lipid-H } \rightarrow \text { lipid }+\mathrm{CHCl}^{-}
\end{aligned}
$$$$
\mathrm{CHCl}_{3}+[\mathrm{O}] \mathrm{Cyt} \mathrm{P} 450 \rightarrow \mathrm{OCCl} 2 \text { (Covalent binding to macromolecules) }
$$

The heme released from lysed cells due to radical formation through biotransformation of $\mathrm{CCl}_{4}$ can enhance the damage by interacting with more macromolecules, as described above in the discussion of lead poisoning. Again, Hx plays 
the role of an antioxidant by safely delivering the heme to the lysozomes of the liver and therefore reducing lipid peroxidation.

\section{HEMOPEXIN AS AN ANTIOXIDANT}

It has been demonstrated above that certain pollutants can result in severe damage at the cellular level. Serum albumin, transferrin, and $\mathrm{Hx}$ are the major antioxidants of the extracellular transport system that inhibit the catalysis of oxidation of lipids and proteins (Halliwell and Gutteridge, 1990). Serum albumin and Hx are both able to bind heme, with the albumin serving as the primary scavenger and $\mathrm{Hx}$ as the transporter. The issue of heme delivery to a specific site in the liver has been controversial. On the basis of experiments with cultured cells, the assertion has been made that albumin may be the primary agent for delivery to a hepatocyte heme receptor (Muller-Eberhard and Nikkilä, 1989), and that although plasma Hx concentrations are lowered after intravenous injection of large amounts of heme (Lane et al., 1977; Wochner et al., 1974), the heme bound to Hx may not reach hepatocytes, as does heme bound to serum albumin (Sinclair et al., 1988). On the other hand, a heme $\mathrm{Hx}$ receptor has been reported for rat hepatocytes (Smith and Morgan, 1981); also, more recent work characterizes the receptor (Morgan et al., 1988).

It has been found that $\mathrm{Hx}$ is not cleared from the plasma as efficiently as heme $\mathrm{Hx}$, implying that conformational changes may take place upon binding of heme as seen by CD (Muller-Eberhard and Grizzuti, 1971; Morgan and Muller-Eberhard, 1976). Hx has a two-domain structure; it has been proposed that upon heme binding to domain I, the whole structure undergoes a conformation change which is necessary for receptor recognition (Heiny et al., 1986; Takahashi et al., 1985; Morgan and Smith, 1984; Spencer et al., 1990). This is illustrated in Figure 2. 


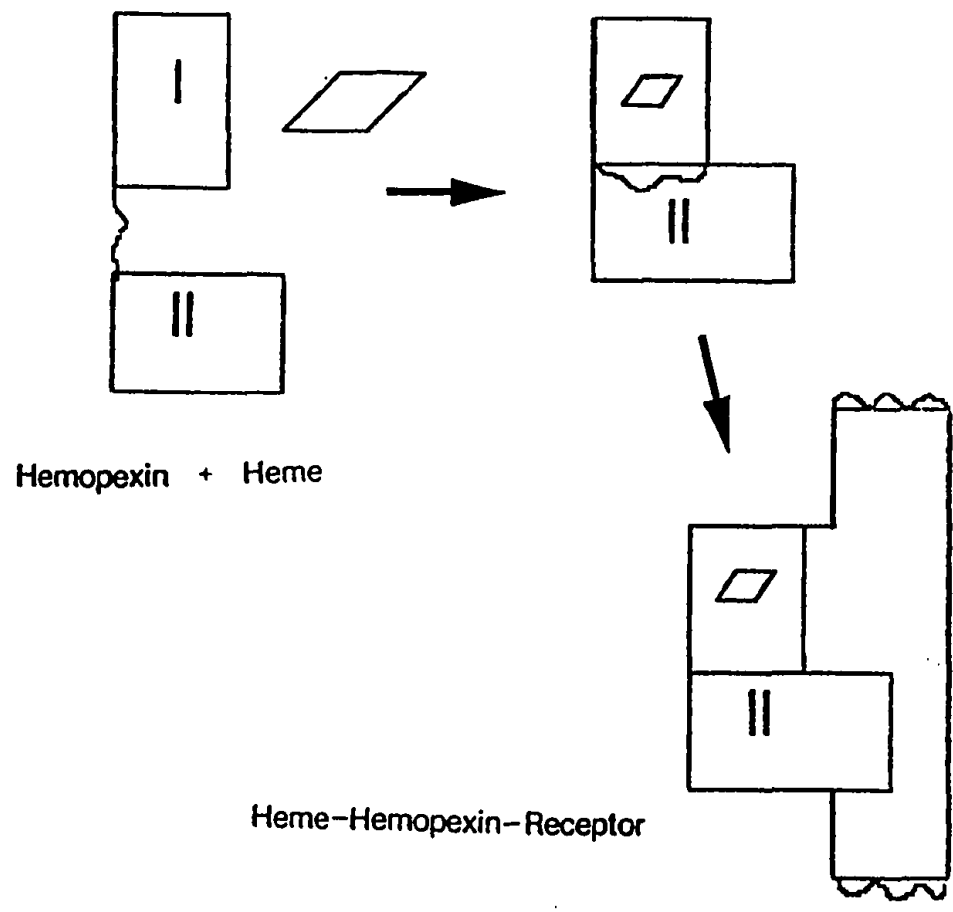

Eigure 2. Schematic representation of conformational change occurring in $\mathrm{Hx}$ upon heme binding. Heme binds to domain I and undergoes a conformational change producing a more compact form, compatible with a receptor site.

\section{RESEARCH APPROACH}

NMR spectroscopy is a powerful tool for delineating structures of protein complexes. The following chapters presented in this dissertation represent an attempt to understand the structure and function of Mb and Hx upon binding to SnPP and heme. Detecting and identifying conformational shifts in a protein complex can be very useful in understanding its function, as well as its interaction with various drugs. The strategy has been to first, resolve the structure of SnPP.EqMb at equilibium, then look into the various species obtained during the reconstitution process, then, to apply the knowledge obtained from SnPP.EqMb to the more difficult and not so well understood Hx. Thus the structures studied are therefore SnPP.Mb and heme-Hx. 


\section{CHAPTER II}

\section{OPTICAL SPECTROSCOPY AND NUCLEAR MAGNETIC RESONANCE SPECTROSCOPY INSTRUMENTATION}

Optical spectroscopy was performed on a Cary 16 spectrophotometer. A Macintosh Plus computer was interfaced to the spectrophotometer in order to efficiently collect and visualize the data. A Keithely model 195A digital volt meter as the ADC and Mac488A bus controller from IOTech were used in collecting the data. Each optical run consisted of 2000 data points acquired over the ranges 750 to $351 \mathrm{~nm}$ in ten minutes $(0.2 \mathrm{~nm}$ digital resolution) or 470 to $337 \mathrm{~nm}$ in three minutes $(0.067 \mathrm{~nm}$ digital resolution). Data smoothing was performed as necessary by applying either a three or a seven point sliding window average. Plots were executed on a Hewlett-Packard model 7470A plotter.

${ }^{1}$ H NMR spectra were recorded on a Bruker AMX-400 NMR spectrometer equipped with a dedicated $5 \mathrm{~mm}{ }^{1} \mathrm{H}$ probe at $25^{\circ} \mathrm{C}$ unless otherwise specified, operating at 400.14 MHz. Two-dimensional phase-sensitive NOESY (nuclear Overhauser effect spectroscopy) (Kumar et al., 1980) is a technique generally applied to biological molecules. It provides information on how far in space the nuclei are separated from each other ( $5 \AA$ or less), as well as the velocity of the internuclear vector (rate of molecular tumbling). The pulse sequence used is:

$$
\pi / 2-t_{1}-\pi / 2-\tau_{m}-\pi / 2-\text { Acquire }\left(t_{2}\right)
$$

One-dimensional NOE techniques provide similar information as NOESY. The difference is in the selective irradiation of a peak of choice, then observing perturbations in adjacent nuclei (other peaks in the spectrum). In NOESY all protons are irradiated at once, none of the pulses are selective. The one-dimensional NOE pulse sequence used is: 


$$
\begin{gathered}
\pi\left(\text { selective inversion) }-\tau_{m}-\pi / 2-\text { Acquire }\left(t_{2}\right)\right. \\
\text { or } \\
\text { (selective saturation) } \tau_{m}-\pi / 2-\text { Acquire }\left(t_{2}\right)
\end{gathered}
$$

Two-dimensional ROESY (rotating-frame Overhauser effect spectroscopy) (Bax and Davis, 1985; Bothner-By et al., 1984) is similar to NOESY, except that the mixing time $\left(\tau_{m}\right)$ is done with a spin-locking field. The advantage of ROESY is that all correlations (NOEs) are positive, regardless of molecular tumbling (molecule size). In this case, intermediate-size molecules which give small or no NOEs can give positive NOEs in the rotating frame, and thus be observed. The pulse sequence used for ROESY is:

$$
\pi-\mathrm{t}_{1}-\pi / 2-\mathrm{SL}\left[\tau_{\mathrm{m}}\right]-\pi / 2-\text { Acquire }\left(\mathrm{t}_{2}\right)
$$

DQF-COSY (double-quantum filtered-correlation spectroscopy) (Marion and Wüthrich, 1983; Rance et al., 1983) is a technique that provides information about spin coupling networks within a molecule (three bonds or less). This is very useful in identifying certain amino acids from their unique spin systems. There are times however when expected cross-peaks are weak or not observed. This is due to cancellation of the positive and negative components characteristic of COSY. The pulse sequence used is:

$$
\pi / 2-\mathrm{t}_{1}-\pi / 2-\text { Acquire }\left(\mathrm{t}_{2}\right)
$$

TOCSY (total correlation spectroscopy) or HOHAHA (homonuclear HartmanHahn) is a two-dimensional experiment in the rotating frame (Braunshweiler and Ernst, 1983) with an MLEV-17 spin-locking sequence (Bax and Davis, 1985). This technique is similar to COSY in the type of information it provides. However, TOCSY gives total relay such that cross peaks arise between all nuclei of a spin system, rather than just individual direct correlations. This technique is a wonderful accompaniment to COSY for confirmation of a spin system in question. However, TOCSY gives negative cross peaks so that negative cross relaxation peaks generated from spin-locking (ROESY; discussed above) can result in overall cancellation. The puise sequence used is: 


$$
\pi / 2-\mathrm{t}_{1}-\operatorname{SL}[\pi / 2(\mathrm{x})-\Delta-\pi(\mathrm{y})-\Delta-\pi / 2(\mathrm{x})]_{16}-\pi / 2-\text { Acquire }\left(\mathrm{t}_{2}\right)
$$

Two-quantum spectroscopy (Braunshweiler et al., 1983) is a technique that includes editing of ${ }^{1} \mathrm{H}$ NMR spectra into subspectra devoid of spin systems with three protons by use of two-quantum spectroscopy. This is a confirmation technique used in establishing unambiguous amino acid connectivities. The pulse sequence used is:

$$
\pi / 2-\tau / 2-\pi-\tau / 2-\pi / 2-\mathrm{t}_{1}-\pi / 2-\text { Acquire }\left(\mathrm{t}_{2}\right)
$$

All two-dimensional experiments were carried out with TPPI (Marion and Wüthrich, 1983) to obtain quadrature detection in $t_{1}$.

${ }^{119} \mathrm{Sn}$ spectra were also collected as free induction decays on the AMX-400 NMR, with a $22.37 \mathrm{KHz}$ bandwidth at $149.12 \mathrm{MHz}$ using a $10 \mathrm{~mm}$ broadband probe at $25^{\circ} \mathrm{C}$, then multiplied by an exponential function to yield $5 \mathrm{~Hz}$ line broadening before Fourier transformation. ${ }^{1} \mathrm{H}$-decoupling, during ${ }^{119} \mathrm{Sn}$ acquisition only, was accomplished using the WALTZ sequence. WALTZ is a very efficient, low power, broadband decoupling sequence (Shaka et al., 1983); low power decoupling and long recycle times (6 s) were used to avoid sample heating.

The manufacturer's pulse sequences were used as supplied. For two-dimensional experiments, 400 - $512 \mathrm{t}_{1}$ values were used, and free induction decays in $\mathrm{t}_{2}$ were recorded in 2048 complex-point blocks, summing either 64 or 128 acquisitions per $t_{1}$. The NOESY mixing time was $100 \mathrm{~ms}$, and 1-dimensional saturation-transfer by chemical exchange experiments had irradiation times up to $4 \mathrm{~s}$. For these spectra, at least 64 scans were accumulated for each irradiation frequency, cycling through the decoupling list after each 8 or 16 scans, and $0.3-4 \mathrm{~Hz}$ line-broadening factors were applied before Fourier transformation. TOCSY mixing times ranged from 26 to $96 \mathrm{~ms}$, and two-quantum spectroscopy mixing times were either 25 or $100 \mathrm{~ms}$. Solvent suppression, to avoid over-filling the 16-bit ADC, was achieved by saturation during the relaxation delay, and the $90^{\circ}$ transmitter pulse was $\sim 9 \mu$ s. The data sets were zero-filled to $1024 \times 1024$ or 
$2048 \times 2048$ data point sets after digital filtering with a $30^{\circ}$ to $45^{\circ}$-phase-shifted sinebell-squared function in both dimensions, except for DQF-COSY which were digitally filtered with a $22.5^{\circ}$-phase-shifted sine-bell-squared function. Chemical shifts for all ${ }^{1} \mathbf{H}$ spectra were referenced to 2,2-dimethyl-2-silapentane-5-sulfonate (DSS) through the residual water resonance at $4.76 \mathrm{ppm}$. All spectra were processed on the X-32 computer supplied with the spectrometer, using the manufacturer's software package, UXNMR. 


\section{CHAPTER III}

\section{PREPARATION, ISOLATION, AND PURIFICATION TECHNIQUES FOR APOMYOGLOBIN FROM HORSE HEART, AND HEMOPEXIN FROM VARIOUS SPECIES (COW, RAT, HUMAN, RABBIT)}

\section{PREPARATION OF EQUINE APOMYOGLOBIN}

Equine myoglobin (EqMB) was purchased from Sigma. ApoEqMb was then obtained by the method of (Teale, 1959), that relies on organic extraction of the heme after acidification of the myoglobin solution, which causes protonation of the heme propionates. Thus, approximately 1 gram of $\mathrm{Mb}$ was dissolved in $50 \mathrm{~mL}$ of distilled water at $5^{\circ} \mathrm{C}$. This mixture was then acidified with $0.96 \mathrm{M} \mathrm{HCl}$ until the $\mathrm{pH}$ of the mixture was 2.66 . The acidic mixture was then extracted gently with $4 \times 50 \mathrm{~mL}$ of methyl ethyl ketone (MEK). The aqueous layer containing the apoMb was separated from the dark organic layer containing the heme. The apoMb solution was added to presoaked dialysis tubing (Spectrapor membrane tubing, MW cutoff: 6-8 KDa) and dialyzed against distilled water for 24 hours, with four exchanges of $4 \mathrm{~L}$ of water every 6 hours. The apoMb solution was then dialyzed against $0.05 \mathrm{M}$ phosphate buffer for 8 hours, with four exchanges of $2 \mathrm{~L}$ of buffer every 2 hours. The apoMb solution was again dialyzed against distilled water overnight. The apoMb solution was filtered to separate the precipitate from the supernatant. The supernatant containing the apoMb was then frozen in a lyophilizing jar by slowly rotating the jar in a mixture of dry ice-acetone. The protein was lyophilized and the resulting apoMb was weighed and stored in a tightly sealed jar at $-20^{\circ} \mathrm{C}$. 


\section{PREPARATION OF HEME-AGAROSE COLUMN}

This technique was reported previously (Muller-Eberhard, 1988; Tsutsui and Mueller, 1982). $\omega$-Aminoethyl agarose (100 mL) was washed with a) $2 \mathrm{~L}$ of distilled water, b) $0.5 \mathrm{~L}$ of $33 \%$ Dimethylformamide (DMF), c) $0.5 \mathrm{~L}$ of $66 \% \mathrm{DMF}$ and, d) $1 \mathrm{~L}$ of $100 \%$ DMF. Bovine heme from Sigma (1.6 g) was dissolved in $150 \mathrm{~mL}$ DMF. $2 \mathrm{~g}$ of $1,1^{\circ}$-carbonyldiimidazole (CDI) were added to the heme mixture and heated to $80^{\circ} \mathrm{C}$ for $30 \mathrm{~min}$. The heme mixture was then cooled to room temperature and added to the gel mixture. The combination was incubated for 18 hours while gently shaking. The brownish colored beads were washed with a) $1 \mathrm{~L}$ of $100 \% \mathrm{DMF}$ b) $0.5 \mathrm{~L}$ of $66 \% \mathrm{DMF}$, c) $0.5 \mathrm{~L}$ of $33 \% \mathrm{DMF}$, and d) $2 \mathrm{~L}$ of distilled water. This process eliminates excess (unbound) heme. The heme-agarose gel was washed with $2 \mathrm{~L}$ pyridine until the color of the wash turned from light brown to clear. The gel was then washed with $2 \mathrm{~L}$ of distilled water until the odor of pyridine was eliminated.

The gel was loaded onto a column $(1 \times 20 \mathrm{~cm})$ and equilibrated with a $\mathrm{pH} 7.5$ buffer I (10 mM phosphate, $0.1 \mathrm{M} \mathrm{NaCl}, 1 \mathrm{mM}$ EDTA [ethylene diamine tetra acetic acid], $0.02 \%$ sodium azide, $10^{-4} \mathrm{M}$ PMSF [a-toluenesulfonyl flouride], $1 \mathrm{mM}$ DTT [dldithiothreitol], $5 \mathrm{mg} / \mathrm{ml}$ aprotinin, $1 \mathrm{mg} / \mathrm{ml}$ leupeptin, $1 \mathrm{mg} / \mathrm{ml}$ antipain).

\section{ISOLATION AND PURIFICATION OF HEMOPEXIN (HX) FROM BOVINE PLASMA WITH HEME-AGAROSE}

This isolation and purification technique was reported previously (Muller-Eberhard, 1988; Tsutsui and Mueller, 1982). Bovine plasma (100 mL) was quickly defrosted, and $2.5 \mathrm{mg}$ of aprotinin and $100 \mathrm{~mL}$ of $10^{-4} \mathrm{M}$ PMSF were added. The mixture was chilled for 2-2.5 hrs, then dialyzed against buffer I for 2 days . Following dialysis, the plasma 
was diluted with $200 \mathrm{~mL}$ buffer I and centrifuged at $2000 \mathrm{~g}$ for $5 \mathrm{~min}$, by using a Sorvall superspeed model RC2-B centrifuge, to get rid of the pelleted fat.

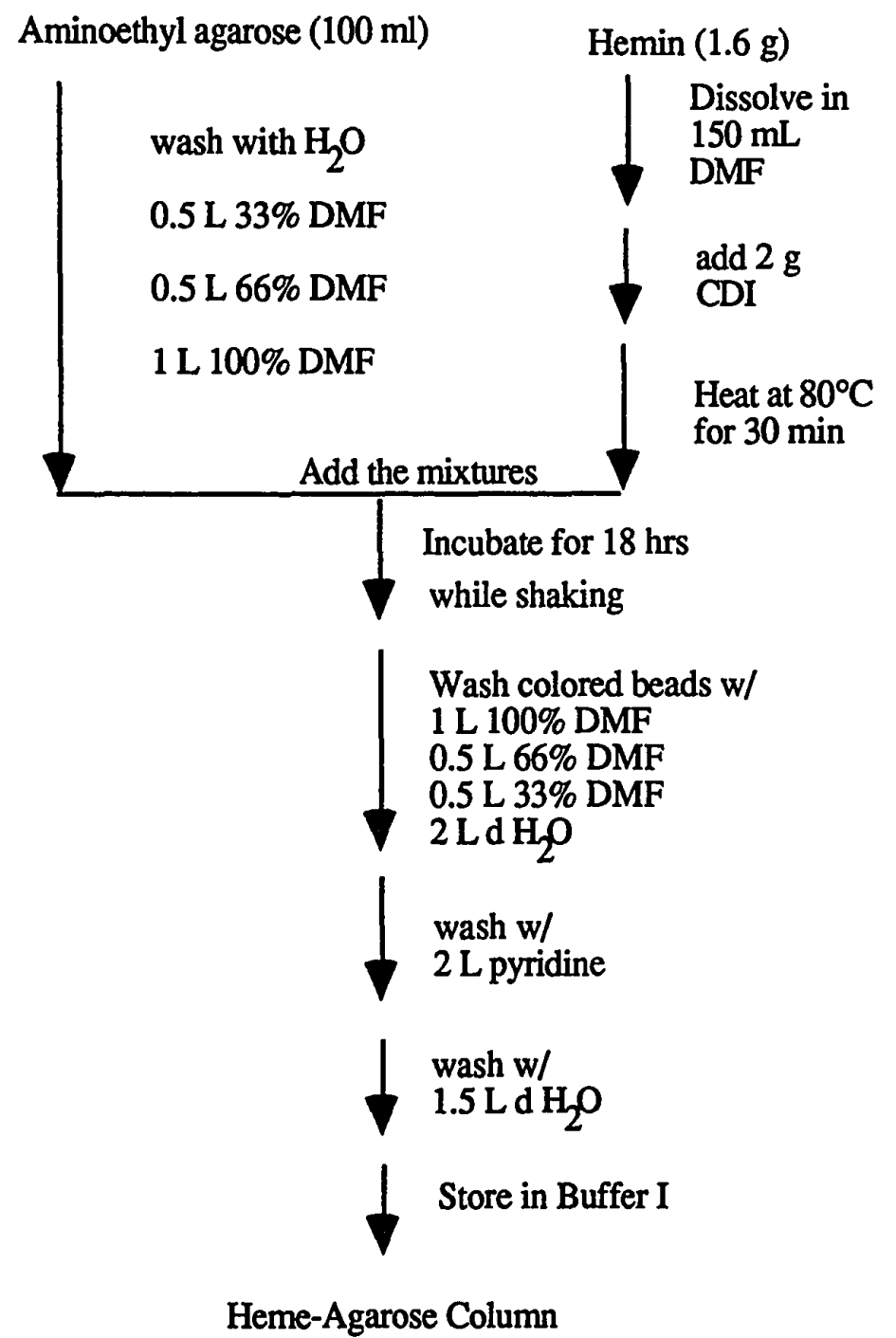

Figure 3. Schematic diagram for the preparation of Heme-Agarose column.

The plasma was then loaded onto the pre-equilibrated heme-agarose column and eluted with buffer II at pH 7.5 ( $10 \mathrm{mM} \mathrm{Pi,} 0.5 \mathrm{M} \mathrm{NaCl}, 1 \mathrm{mM}$ EDTA, $0.02 \%$ sodium azide, $1 \mathrm{mM}$ DTT, $10^{-4} \mathrm{M}$ PMSF, $5 \mathrm{mg} / \mathrm{mL}$ aprotinin, $1 \mathrm{mg} / \mathrm{mL}$ leupeptin, $1 \mathrm{mg} / \mathrm{mL}$ 
antipain). This process strips the column from everything except $\mathrm{Hx}$ from the heme-agarose gel. After the breakthrough fraction, the $\mathrm{Hx}$ was stripped off the column with buffer III at pH 2.8 ( $50 \mathrm{mM}$ citric acid, $50 \mathrm{mM}$ succinic acid, $0.5 \mathrm{M} \mathrm{NaCl}, 0.02 \%$ sodium azide). This process was set up on an ISCO model 1133 fraction collector Model 1133 such that $2 \mathrm{~mL}$ fractions were collected, each tube containing $100 \mathrm{~mL}$ of $1 \mathrm{M}$ Tris, pH 9. This process took place over 1 hour.

The fractions containing $\mathrm{Hx}$ were pooled and dialyzed against buffer $\mathrm{V}$ at $\mathrm{pH} 5.2$ ( 20 $\mathrm{mM}$ sodium acetate, $50 \mathrm{mM} \mathrm{NaCl}, 1 \mathrm{mM}$ EDTA) until the $\mathrm{pH}$ of the protein was raised to 5.2. This was followed by dialysis against $1 \mathrm{~L} 50 \mathrm{mM} \mathrm{Pi}, 100 \mathrm{mM} \mathrm{NaCl}$ at $\mathrm{pH} 7.15$, and then $2 \mathrm{~L} 10 \mathrm{mM} \mathrm{Pi}, 100 \mathrm{mM} \mathrm{NaCl}$ at $\mathrm{pH} 7.2$.

\section{ISOLATION AND PURIFICATION OF HEMOPEXIN FROM SERUM BY ION EXCHANGE}

$\mathrm{Hx}$ was isolated from serum by modification of a previously reported procedure (Noiva et al., 1987). Plasma (50 mL) was mixed with an equal volume of saturated ammonium sulfate, then left stirring at $25^{\circ} \mathrm{C}$ for one hour. The plasma was then centrifuged at $12000 \mathrm{~g}$ at $4^{\circ} \mathrm{C}$ for one hour. The precipitate was discarded and the supernatant was dialyzed against distilled water for 18 hours, followed by dialysis against $0.01 \mathrm{M}$ sodium citrate at $\mathrm{pH} 5.9$ for another 18 hours (three changes). The supernatant was then concentrated to $15 \mathrm{~mL}$ over an Amicon YM-30 ultrafiltration membrane and loaded onto a CM-Sepharose column $(1.5 \times 45 \mathrm{~cm})$ pre-equilibrated with $0.01 \mathrm{M}$ sodium citrate at $\mathrm{pH}$ 5.9. The column was eluted with the same buffer. Following the breakthrough fraction (containing serum albumin), an ion-exchange gradient ( $300 \mathrm{~mL}$ of $0.01 \mathrm{M}$ to $300 \mathrm{~mL}$ of $0.1 \mathrm{M}$ sodium citrate) at $\mathrm{pH} 5.9$ was set up. This ion-exchange step separates transferrin from hemoglobin $(\mathrm{Hb})$ and $\mathrm{Hx}$ which co-elute. Fractions were 
collected on a Gilson Microfractionator at a rate of $7 \mathrm{~mL} / 30 \mathrm{~min}$ such that each fraction contained $7 \mathrm{~mL}$. The fractions were tested for heme-binding by checking the absorption spectra in the visible and the Soret regions.

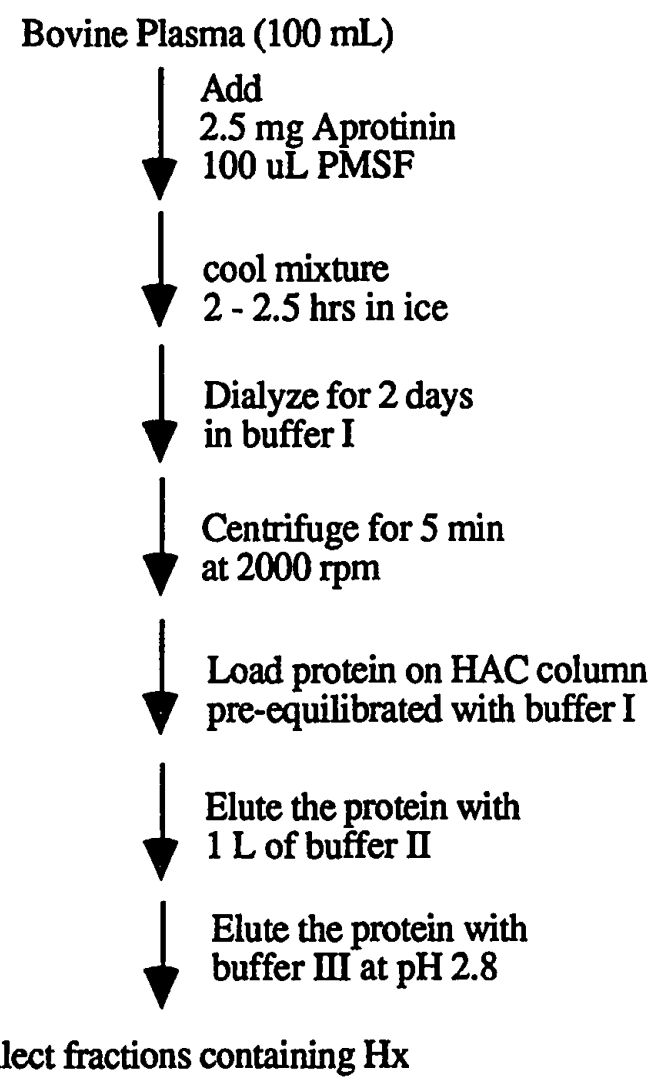

Figure 4. Schematic diagram for the isolation of hemopexin by the Heme-Agarose method.

\section{RECONSTITUTION OF HEMOPEXIN WITH PROTOHEMIN IX}

The heme-binding fractions that eluted from the CM-Sepharose column were combined and reconstituted with excess heme, then incubated at $5^{\circ} \mathrm{C}$ for 1 hour. Excess heme was removed by passing the mixture through a small Sephadex DEAE-column ( 0.5 $x 5 \mathrm{~cm}$ ). The column was pre-equilibrated with $50 \mathrm{mM}$ phosphate and $100 \mathrm{mM} \mathrm{NaCl}$ at $\mathrm{pH}$ 7.3. The reconstituted protein was then concentrated to $5 \mathrm{~mL}$ via $\mathrm{YM}-30$ and loaded 
onto a Sephacryl S-200 column $(95 \times 2.5 \mathrm{~cm})$ pre-equilibrated with a pH 7.3 buffer (50 $\mathrm{mM}$ phosphate, $100 \mathrm{mM} \mathrm{NaCl}$ ). This gel exclusion step separates the first band $(\mathrm{Hb})$ from the second band $(\mathrm{Hx})$. The fraction collector was set up to deliver $7 \mathrm{~mL} /$ fraction every $30 \mathrm{~min}$. The fractions containing $\mathrm{Hx}$ were tested by the appearance of the visible and the Soret regions of the absorption spectra.

Electrophoresis (SDS-PAGE) was performed in a $0.75 \mathrm{~mm} \times 8 \mathrm{~cm}$ slab gel containing $10 \%$ polyacrylamide. The protein fractions were treated with $10 \% \beta$-mercaptoethanol and $20 \%$ glycerol for $90 \mathrm{~s}$ at $100^{\circ} \mathrm{C}$. The samples were cooled and applied to wells in the stacking gel phase at a protein concentration of up to $20 \mathrm{mg} /$ well. Electrophoresis was carried out at $25^{\circ} \mathrm{C}$ at a constant voltage of $100 \mathrm{~V}$. Gels were stained with Coomassie Brilliant blue. Molecular masses were estimated on the basis of the $R_{\mathbf{f}}$ values of known proteins from $18 \mathrm{KDa}$ (Myoglobin) to $60 \mathrm{KDa}$ (serum albumin).

The fractions containing Hx॰heme were pooled, exchanged into deuterated buffer ( 50 $\mathrm{mM}$ phosphate and $100 \mathrm{mM} \mathrm{NaCl}$ ) at $\mathrm{pH} 7.5$, and then concentrated via $\mathrm{YM} 30$ to a volume of $500 \mu \mathrm{L}$ for NMR experiments (see Chapter VIII). Table I shown below summarizes the attempts used to isolate rabbit $\mathrm{Hx}$ by the "ion-exchange" method. Of these, it was found that modification IV was the best, and was used for all subsequent isolations. Lyophilization was completely omitted from the original method due to the instability of apoHx. Dialysis at pH 5.9 in citrate buffer was completely omitted from modification I as well the G-200 gel separation step. Separation over G-200 was very slow, and exposure of unstable apoHx to a pH 5.9 environment for a long period of time was not ideal. 


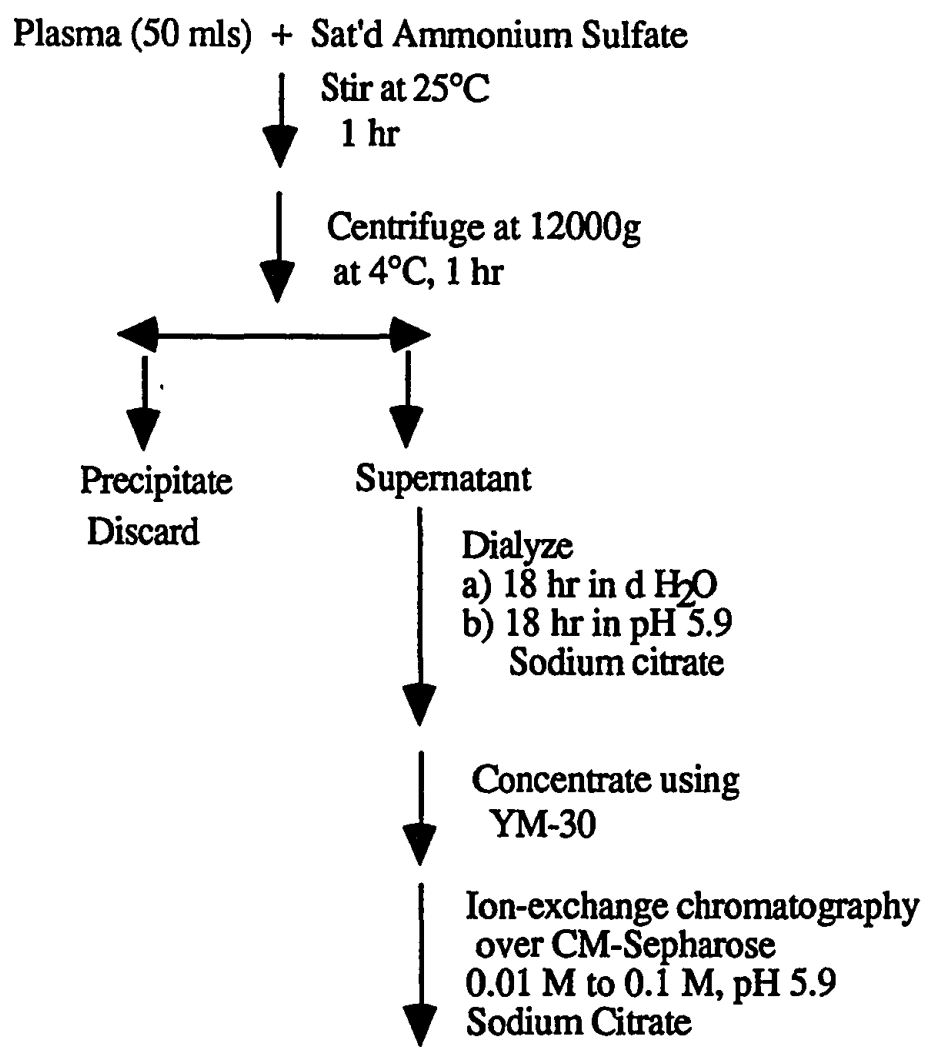

Collect $\mathrm{Hx}$ and $\mathrm{Hb}$ Fractions

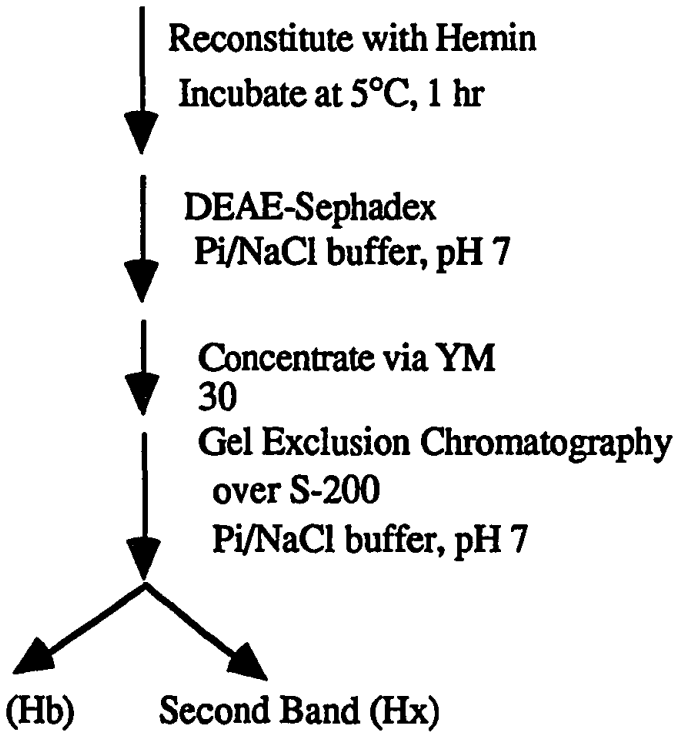

Figure 5. Schematic diagram for the isolation and purification of hemopexin by a modified technique. Reconstitution of hemopexin with hemin. 
TABLE I

VARIOUS METHODS USED FOR ISOLATING HX FROM PLASMA

\begin{tabular}{lccccc} 
Method & original & Mod I & Mod II & Mod III & Mod IV \\
\hline Precipitation & x & x & x & x & x \\
Centrifugation & x & x & x & x & x \\
Dialysis, H2O & x & x & x & x & x \\
Dialysis, pH 5.9 & x & x & x & x & x \\
CM-Sepharose & x & x & x & x & x \\
Heme Reconstitution & & & x & x & x \\
Dialysis, H2O & x & x & & & \\
Dialysis, pH 5.9 & & x & & & \\
Lyophilization & x & & & & \\
DEAE-Sephadex & & & & & x \\
S-200(Sephacryl), pH 5.9 & & & & & \\
S-200, Pi Buffer, pH 7 & & & & & x \\
G-200 (Sephadex), pH 5.9 & x & x & & & \\
Dialysis, Pi Buffer, pH 7 & & x & x & &
\end{tabular}




\section{CHAPTER IV}

\section{CHARACTERIZATION AND PROPERTIES OF SNPP AND (SNPP)2}

$\mathrm{SnPP}$ is a strong competitive inhibitor of heme oxygenase and medicinally useful in the treatment of hyperbilirubinemia in newborn infants. Currently, a related and slightly more stable porphyrin, mesoporphyrin is in clinical trial for jaundice treatment in newborn infants (G. Drummond, private communication). SnPP is a well behaved porphyrin under varying conditions of $\mathrm{pH}$ and temperature and a good choice for a prosthetic group in the studies of reconstitution kinetics with proteins, and their contributions to protein structure stability. Breslow et al. (1986) studied the properties of SnPP optically. They found that in solution, SnPP behaves as a slowly interconverting monomer-dimer system, such that the equilibrium between them is $\mathrm{pH}$ dependent. This chapter deals with understanding the kinetics of $\mathrm{SnPP}$ and $(\mathrm{SnPP})_{2}$ by optical spectroscopic studies, as well as the characterization of their structure by NMR and Fourier Transform Infrared spectroscopy (FTIR). The material presented in this chapter was included in the publication: Deeb, R.S., and Peyton, D.F. (1992) "Proton NMR Study of the Interaction of Tin(IV)Protoporphyrin IX Monomers and Dimers with Apomyoglobin" Biochemistry 31, 468-474.

\section{MATERIALS AND METHODS}

SnPP was from Porphyrin Products. SnPP solutions were always prepared fresh by dissolving the porphyrin in a minimum amount of base $\left(\mathrm{NaOH}\right.$ or $\left.\mathrm{NaO}^{2} \mathrm{H}\right)$ in ${ }^{1} \mathrm{H}_{2} \mathrm{O}$ or ${ }^{2} \mathrm{H}_{2} \mathrm{O}$ solvent as required. All samples prepared were protected from light, and the 
stability of the samples to light exposure during optical work was verified by obtaining multiple scans on the same sample, whose visible spectrum did not change (Breslow et al., 1986).

Optical spectra were obtained on a Cary 16 spectrophotometer described in Chapter II. Infrared spectra were obtained using a Nicolet DX-20 spectrometer and silver chloride sample holders. The infrared solvent was either ${ }^{2} \mathrm{H}_{2} \mathrm{O}$ or $50: 50{ }^{2} \mathrm{H}_{2} \mathrm{O} /{ }^{1} \mathrm{H}_{2}{ }^{18} \mathrm{O}$ to obtain the best window possible in the region between 500 and $1000 \mathrm{~cm}^{-1}$ (Caughey, 1980).

Two-dimensional NMR experimets are described in Chapter II. ${ }^{1} \mathrm{H}$ spectra were recorded for $\mathrm{SnPP}$ in ${ }^{2} \mathrm{H}_{2} \mathrm{O}$ at $25^{\circ} \mathrm{C}$, pH 11.5, and for (SnPP) ${ }_{2}$ in ${ }^{2} \mathrm{H}_{2} \mathrm{O}$ at $25^{\circ} \mathrm{C}, \mathrm{pH}$ 7.4. ${ }^{13} \mathrm{C}:{ }^{1} \mathrm{H}$-decoupled, ${ }^{13} \mathrm{C}:{ }^{1} \mathrm{H}$-coupled, as well as ${ }^{13} \mathrm{C}$-1 $\mathrm{H}$ inverse detection $\mathrm{NMR}$ spectroscopy (Shaka et al., 1983), using a ${ }^{13} \mathrm{C} /{ }^{1} \mathrm{P} /{ }^{19} \mathrm{~F} /{ }^{1} \mathrm{H}$ "quad" probe, were performed on SnPP in ${ }^{2} \mathrm{H}_{2} \mathrm{O}$ at $25^{\circ} \mathrm{C}$, pH 11.5 . ${ }^{119} \mathrm{Sn}$ spectroscopy was performed with a $10 \mathrm{~mm}$ broadband probe as described in Chapter II for both SnPP and (SnPP) 2 in the solvents described above.

\section{RESULTS}

\section{$\mathrm{SnPP}$ and $(\mathrm{SnPP})_{2}$ - $\mathrm{H}_{\mathrm{H}}$ NMR Resonance Assignments}

Figure 6 shows the intersubstituent NOEs expected for a porphyrin ring. Assignments were obtained for all SnPP resonances, with the NOEs between the meso $\mathrm{H}_{\delta}$ at $10.83 \mathrm{ppm}$ and both the $1 \mathrm{CH}_{3}$ at $3.94 \mathrm{ppm}$ and $8 \mathrm{CH}_{3}$ at $3.85 \mathrm{ppm}$ as the starting point. Figure 7 shows the two-dimensional ROESY spectrum of SnPP at pH 11.5. The $1 \mathrm{CH}_{3}$ shows an NOE to $2 \mathrm{H}_{\beta t}$ at $6.66 \mathrm{ppm}$, which in turn shows an NOE to $2 \mathrm{H}_{\beta \mathrm{c}}$ at $6.42 \mathrm{ppm}$. $2 \mathrm{H}_{\alpha}$ shows an NOE to $2 \mathrm{H}_{\beta c}$ at $8.59 \mathrm{ppm}$ which also shows an $\mathrm{NOE}$ to meso- $\mathrm{H}_{\alpha}$ at 10.88 ppm. A NOE is seen from meso- $\mathrm{H}_{\alpha}$ to $3 \mathrm{CH}_{3}$ at $3.90 \mathrm{ppm}$, which shows an NOE to $4 \mathrm{H}_{\beta} \mathrm{t}$ 
at $6.66 \mathrm{ppm}$. $4 \mathrm{H}_{\beta \mathrm{t}}$ shows an NOE to $4 \mathrm{H}_{\beta \mathrm{c}}$ at $6.42 \mathrm{ppm}$ which in turn shows an NOE to $4 \mathrm{H}_{\alpha}$ at $8.59 \mathrm{ppm}$. The $4 \mathrm{H}_{\alpha}$ shows an NOE to the meso- $\mathrm{H}_{\beta}$ at 10.83 , ppm which in turn shows an $\mathrm{NOE}$ to $5 \mathrm{CH}_{3}$ at $3.82 \mathrm{ppm}$. The $5 \mathrm{CH}_{3}$ shows an $\mathrm{NOE}$ to the $6 \mathrm{H}_{\alpha}$ at $4.59 \mathrm{ppm}$. The $6 \mathrm{H}_{\alpha}$ protons show an $\mathrm{NOE}$ to the meso- $\mathrm{H}_{\gamma}$ at $10.79 \mathrm{ppm}$, which in turn shows an NOE to the $7 \mathrm{H}_{\alpha}$ at $4.59 \mathrm{ppm}$. The $6,7 \mathrm{H}_{\alpha}$ protons show NOEs to the $6,7 \mathrm{H}_{\beta}$ protons at $3.25 \mathrm{ppm}$.

Figure 8 shows the ROESY two-dimensional spectrum of $(\mathrm{SnPP})_{2}$ at $\mathrm{pH} 7.4$. The assignments for $(\mathrm{SnPP})_{2}$ were assigned in a similar fashion as monomeric SnPP. The chemical shifts for SnPP and (SnPP) 2 are summarized in Table II. It is important to note that some fractions of higher-mers are also visible in the NMR spectra, but were not further characterized.

Some of the (SnPP) ${ }_{2}^{1}{ }^{1} \mathrm{NMR}$ resonances are split. The most likely explanation for this splitting is that different porphyrin structures are formed (eg., face-to-face and face-to-back), which are not in fast exchange. Additional splitting of the propionate proton resonances can arise due to inequivalence of geminal protons from restricted rotation within the propionates. Each meso-proton resonance is ring-current-shifted upfield by $1.04+0.02 \mathrm{ppm}$ in $(\mathrm{SnPP})_{2}$ relative to $\mathrm{SnPP}$. This is indicative of a cofacial structure (Johnson and Bovey, 1958), rather than the more usual case of porphyrin aggregation in which there is substantial offset of the two porphyrin rings (Hunter and Sanders, 1990; Abraham and Smith, 1983). The upfield shift of each meso-proton from SnPP upon dimerization is almost identical to the ring-current-induced shift of the $\mu$-oxo oligomer [Sc(octaethylporphyrin) $]_{2} \mathrm{O}$ relative to monomeric Sc(octaethylporphyrin)acetate (La Mar et al., 1973). The possibility of a Sn-Sn bond may be eliminated because a much larger upfield shift in the meso resonances would have resulted (Scheer and Katz, 1975). Further evidence for $(\mathrm{SnPP})_{2}$ being a $\mu$-oxo dimer is provided from ${ }^{119} \mathrm{Sn} \mathrm{NMR} \mathrm{experiments.}$ 
TABLE II

${ }^{1} \mathrm{H}$ AND ${ }^{13} \mathrm{C}$ NMR CHEMICALSHIFTS FOR SnPP AND (SnPP) ${ }_{2}$ AT $25^{\circ} \mathrm{Ca}$

\begin{tabular}{llll} 
Assignment & SnPP & $(\mathrm{SnPP})_{2}$ & $13 \mathrm{C}$ SnPPe \\
$\alpha$-meso & 10.88 & 9.86 & 100.9 \\
$\beta$-meso & 10.83 & 9.80 & 100.9 \\
$\gamma$-meso & 10.79 & 9.73 & 100.9 \\
$\delta$-meso & 10.83 & $9.78^{\mathrm{b}}$ & 100.9 \\
$1 \mathrm{CH}_{3}$ & 3.94 & $3.94^{\mathrm{b}}$ & 14.6 \\
$3 \mathrm{CH}_{3}$ & 3.90 & $3.91^{\mathrm{b}}$ & 14.6 \\
$5 \mathrm{CH}_{3}$ & $3.82^{\mathrm{c}}$ & $3.79 \mathrm{~b}$ & 13.6 \\
$8 \mathrm{CH}_{3}$ & $3.85^{\mathrm{c}}$ & $3.83^{\mathrm{b}}$ & 13.6 \\
$2 \mathrm{H}_{\alpha}$ & 8.59 & 8.58 & 131.9 \\
$2 \mathrm{H}_{\beta c}$ & 6.42 & 6.66 & 125.6 \\
$2 \mathrm{H}_{\beta \mathrm{t}}$ & 6.66 & 6.80 & 125.6 \\
$4 \mathrm{H}_{\alpha}$ & 8.59 & 8.58 & 131.9 \\
$4 \mathrm{H}_{\beta c}$ & 6.42 & 6.66 & 125.6 \\
$4 \mathrm{H}_{\beta \mathrm{t}}$ & 6.66 & 6.80 & 125.6 \\
$6,7 \mathrm{H}_{\alpha, \alpha^{\prime}}$ & 4.59 & $4.63,4.52^{\mathrm{d}}$ & 25.6 \\
$6,7 \mathrm{H}_{\beta}, \beta^{\prime}$ & 3.25 & $3.16,3.05^{\mathrm{d}}$ & 43.0 \\
\hline
\end{tabular}

aSnPP at pH 11.5; (SnPP) 2 at pH 7.5. bThese peaks are split into two peaks in the dimer, chemical shifts are averages. ${ }^{\mathrm{CT}} \mathrm{Th} \mathrm{CH}_{3}$ and $8 \mathrm{CH}_{3}$ assignments may be reversed for SnPP. dThese peaks are very complex multiplets in the dimer, chemical shifts are averages. 'Data obtained from ${ }^{1} \mathrm{H}:{ }^{13} \mathrm{C}$-inverse detection.

\section{${ }^{112} \mathrm{Sn} \mathrm{NMR}$ of $\mathrm{SnPP}$ and ( $\left.\mathrm{SnPP}\right)_{2}$}

${ }^{119} \mathrm{Sn}$ NMR also provided evidence that there is no $\mathrm{Sn}-\mathrm{Sn}$ bond in $(\mathrm{SnPP})_{2}$. Such a structure for $(\mathrm{SnPP})_{2}$ would have a large coupling constant between ${ }^{117} \mathrm{Sn}$ and ${ }^{119} \mathrm{Sn}$ 
(each nucleus is present at $\sim 8 \%$ ); such one-bond couplings are on the order of $1-4.5 \mathrm{KHz}$ (Harris et al., 1978). No such coupling was unambiguously identified. However, small peaks at the putatively much smaller Sn-O-Sn coupling, $\sim 300 \mathrm{~Hz}$, were observed. The presence of multiple ( $\mathrm{SnPP})_{2}$ species leads to broadening of all the peaks, and so the identity of peaks as arising from coupling (each at $\sim 4 \%$ of the major peak) is not certain. The ${ }^{119} \mathrm{Sn}$ chemical shift change on going from SnPP to (SnPP) 2 (observed from a sample containing monomer/dimer mixture) is only $+13.1 \mathrm{ppm}$ seen in Figure 9. This suggests that $\mathrm{Sn}$-coordination number remains the same (six) for these two species (Harris et al., 1978).

\section{${ }^{13}$ C NMR Assignments of SnPP}

The correlation between the known resonances of the ${ }^{1} \mathrm{H}$ NMR spectrum of SnPP and the carbon nuclei was observed by ${ }^{1} \mathrm{H}-{ }^{13} \mathrm{C}$ inverse detection NMR spectroscopy as seen in Figure 10. The carbon chemical shifts for SnPP are listed in Table II.

\section{FTIR for SnPP and (SnPP)2}

An attempt was made to observe changes in the infrared spectrum on going from SnPP to (SnPP)2, which would be indicative of the $\mu$-oxo species. The IR spectrum for $(\mathrm{SnPP})_{2}$ showed a very weak band at $\sim 988 \mathrm{~cm}^{-1}$ which may be due to the $\mathrm{Sn}-\mathrm{O}-\mathrm{Sn}$ asymmetric stretch (Buchler et al., 1973); this band appeared to relocate to $\sim 936 \mathrm{~cm}^{-1}$ when the sample was prepared in 180 -enriched water. This is the isotope effect expected for a $\mu$-oxo metal-O-metal asymmetric stretch (Brown et al., 1969).

\section{Optical Spectroscopic Observation of the Kinetics of $\mathrm{SnPP}$ and $(\mathrm{SnPP})_{2}$}

The rate of dimer dissociation was compared to that of dimer formation. Upon dilution of (SnPP)2, the optical spectra (Figure $11 \mathrm{~B}$ ) clearly showed a drop in intensity of the dimeric peak ( $385.7 \mathrm{~nm}$ ) accompanied by a gain in intensity of the monomeric peak 
$(405.8 \mathrm{~nm})$. The rate of dimer dissociation was found to be $2 \times 10^{-2} \mathrm{~min}^{-1}$ (inset in Figure $11 \mathrm{~B})$. This was unlike the rate of dimer formation $\left(6 \times 10^{-2} \mathrm{~min}^{-1}\right)$ by a factor of four. During the process of dimer formation, a peak developed at $650.0 \mathrm{~nm}$ (inset in Figure $11 \mathrm{~A}$ ) at long time ( $>24 \mathrm{hrs}$ ), which acted independently of the other peaks seen in the optical spectrum upon dilutions. At $\mathrm{pH}$ values $>9$, the $650 \mathrm{~nm}$ peak gained in intensity until it reached a maximum where it stopped changing (Figure $11 \mathrm{~B}$ inset). This peak can be related to the formation of an unknown third species, however, it does not rise from a photochemical reaction since all solutions containing SnPP were protected from light. All attempts to try to reverse the formation of the species at $650 \mathrm{~nm}$ failed.

\section{DISCUSSION}

Evidence from ${ }^{1} \mathrm{H}$ and ${ }^{119} \mathrm{Sn} \mathrm{NMR}$, and infrared spectroscopy about the structure of $(\mathrm{SnPP})_{2}$ are consistent with a $\mu$-Oxo bridged oligomer. The alternative dimer, formed by $\pi-\pi$ interactions (Hunter and Sanders, 1990), would be expected to produce an offset geometry for the highly unsymmetric porphyrin core of SnPP, and this would have produced greatly different shifts among the meso-protons upon dimerization (Abraham et al., 1976; Abraham and Smith, 1983). The evidence from ${ }^{119} \mathrm{Sn}$ NMR indicates that $(\mathrm{SnPP})_{2}$ has two axial ligands; it seems unlikely that the cofacial geometry would be possible without oxygen bridging. The very slow exchange between $\mathrm{SnPP}$ and $(\mathrm{SnPP})_{2}$ (Breslow et al., 1986) would also be unusual for $\pi$ - $\pi$ interaction (Scheer and Katz, 1975; Pasternack et al., 1973). Nevertheless, the conclusion that (SnPP) $)_{2}$ is a $\mu$-oxygen bridged oligomer is tentative until (SnPP) 2 and/or related porphyrins are isolated and characterized more fully. Even if $(\mathrm{SnPP})_{2}$ has an oxygen bridge, it may be protonated as has been postulated for an $\mathrm{Fe}^{\mathrm{III}}$ (tetracarboxyphenylporphyrin) in water solvent (Stong and Hartzell, 1976). 
Kinetic studies from optical spectroscopy support the evidence presented by Breslow et al. (1986) that Sn-protoporphyrin behaves in solution as a slowly interconverting monomer-dimer system which is pH-dependent. Furthermore, it shows that upon dimer formation, a third strongly absorbing irreversible species forms. The assumption that this species forms upon the reduction of the porphyrin to a chlorin is highly unlikely, especially when no reducing agents were present in the solutions used for dissolving the porphyrin. It is important to note that the formation of this third species complicates the potential use $\mathrm{SnPP}$ in medicine. SnPP has to be dissolved fresh just before use, and undergoes irreversible changes within 24 hours.

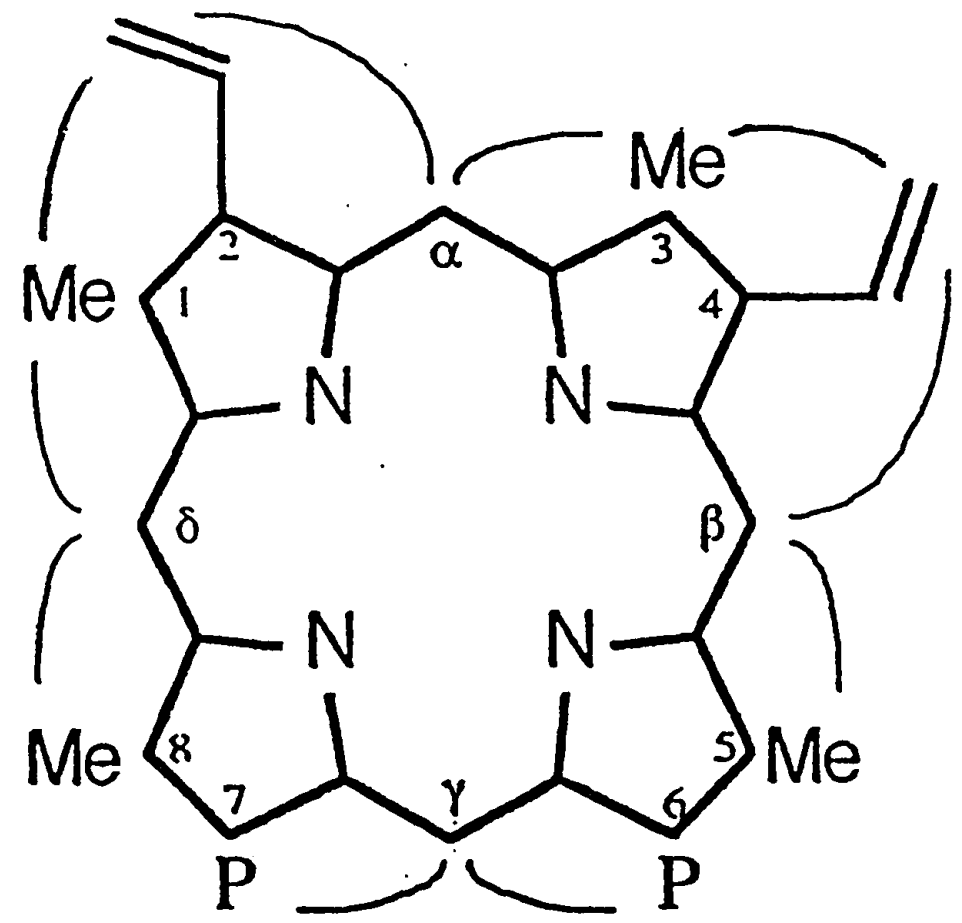

Eigure 6. PPIX skeleton showing intersubstituent NOEs (arcs) observed in ROESY spectra of SnPP and (SnPP)2. 


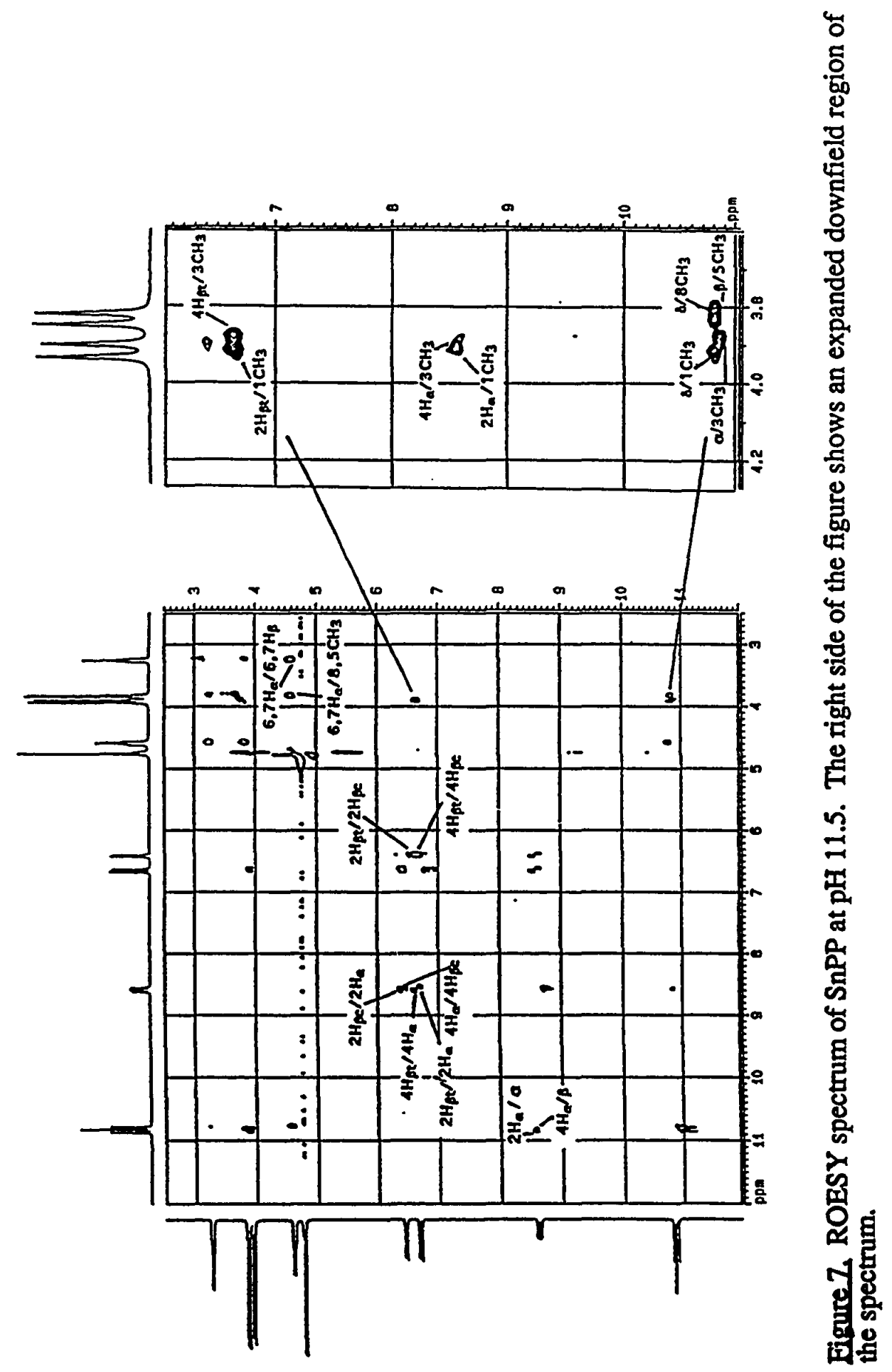




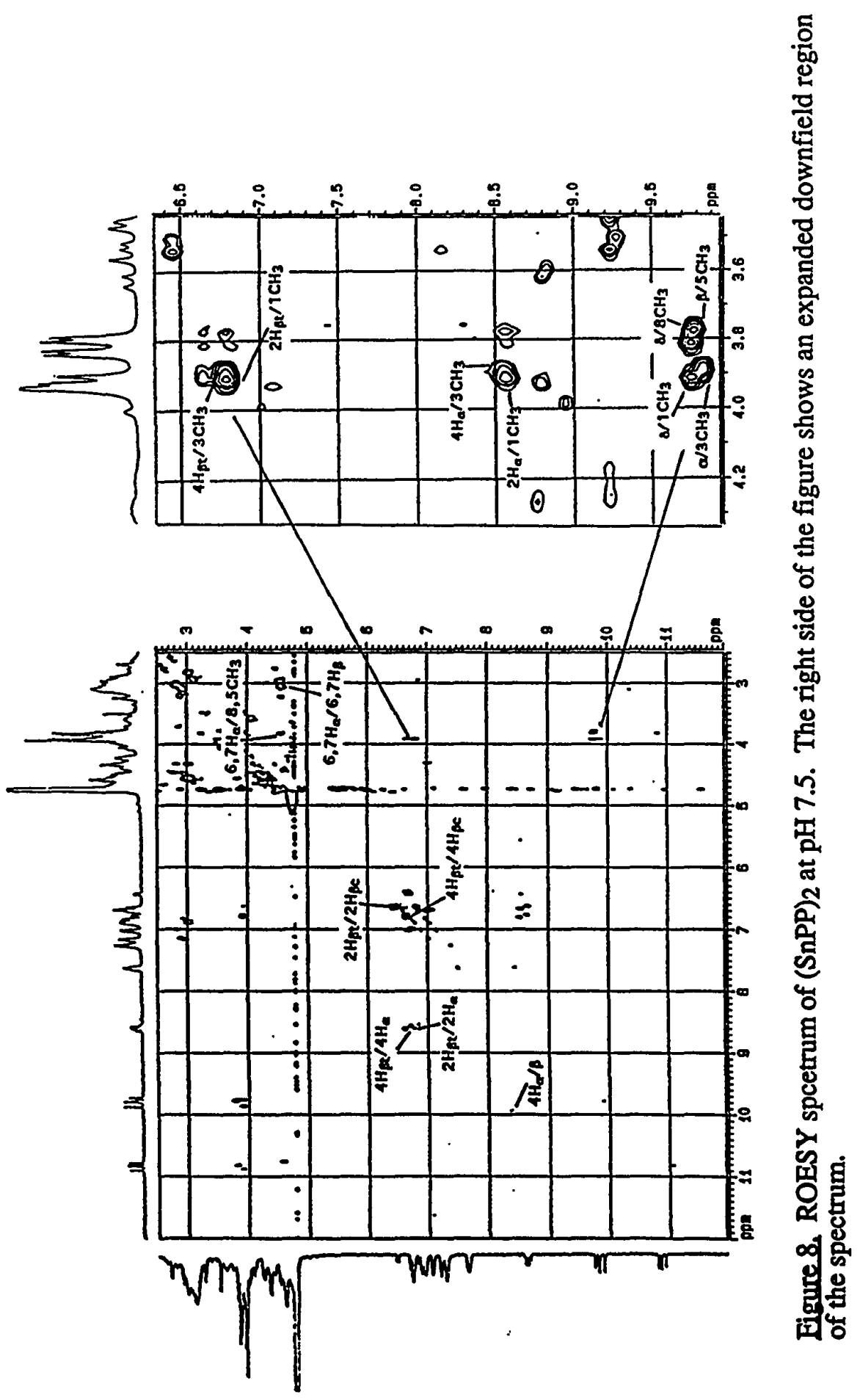




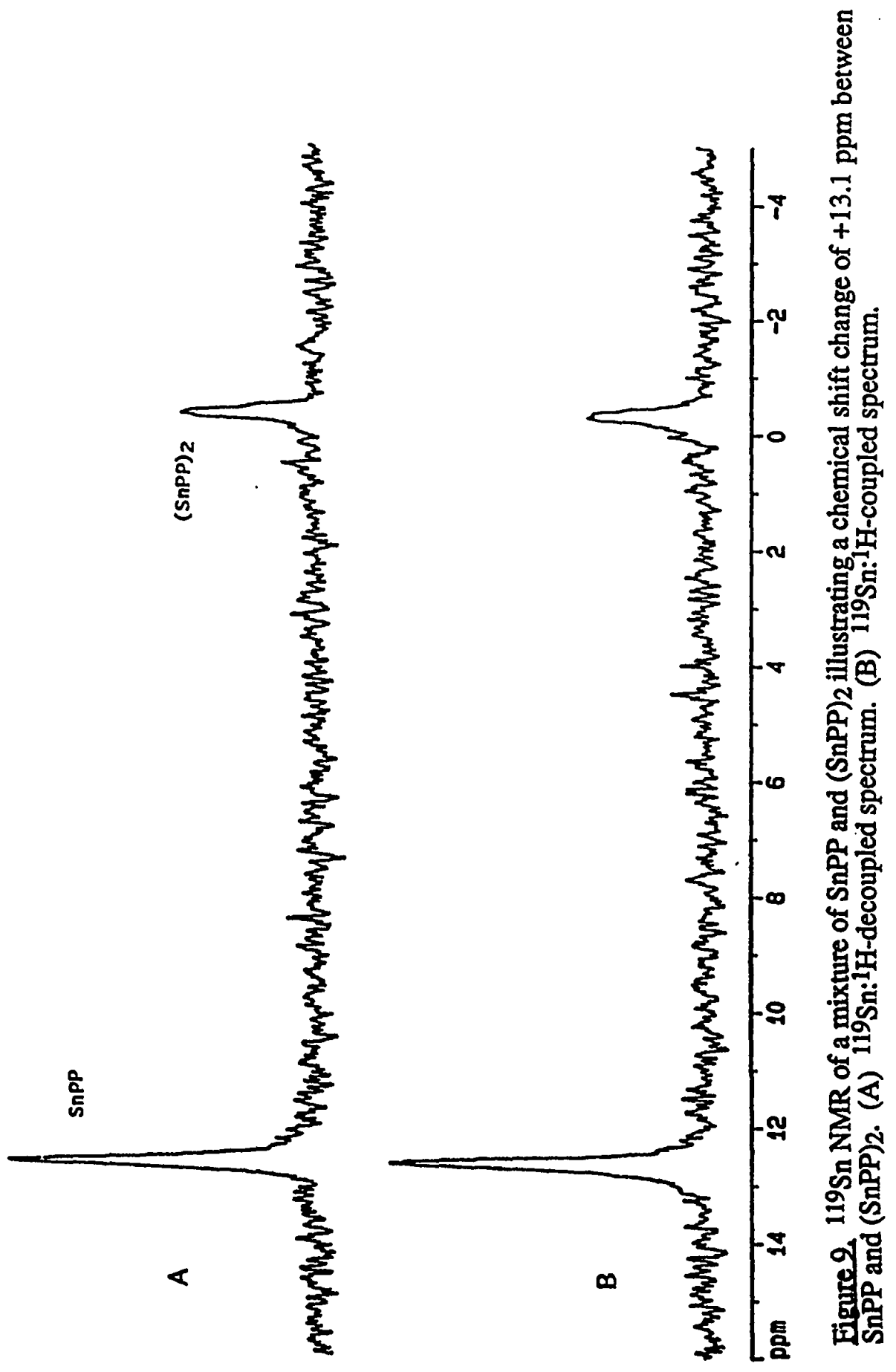




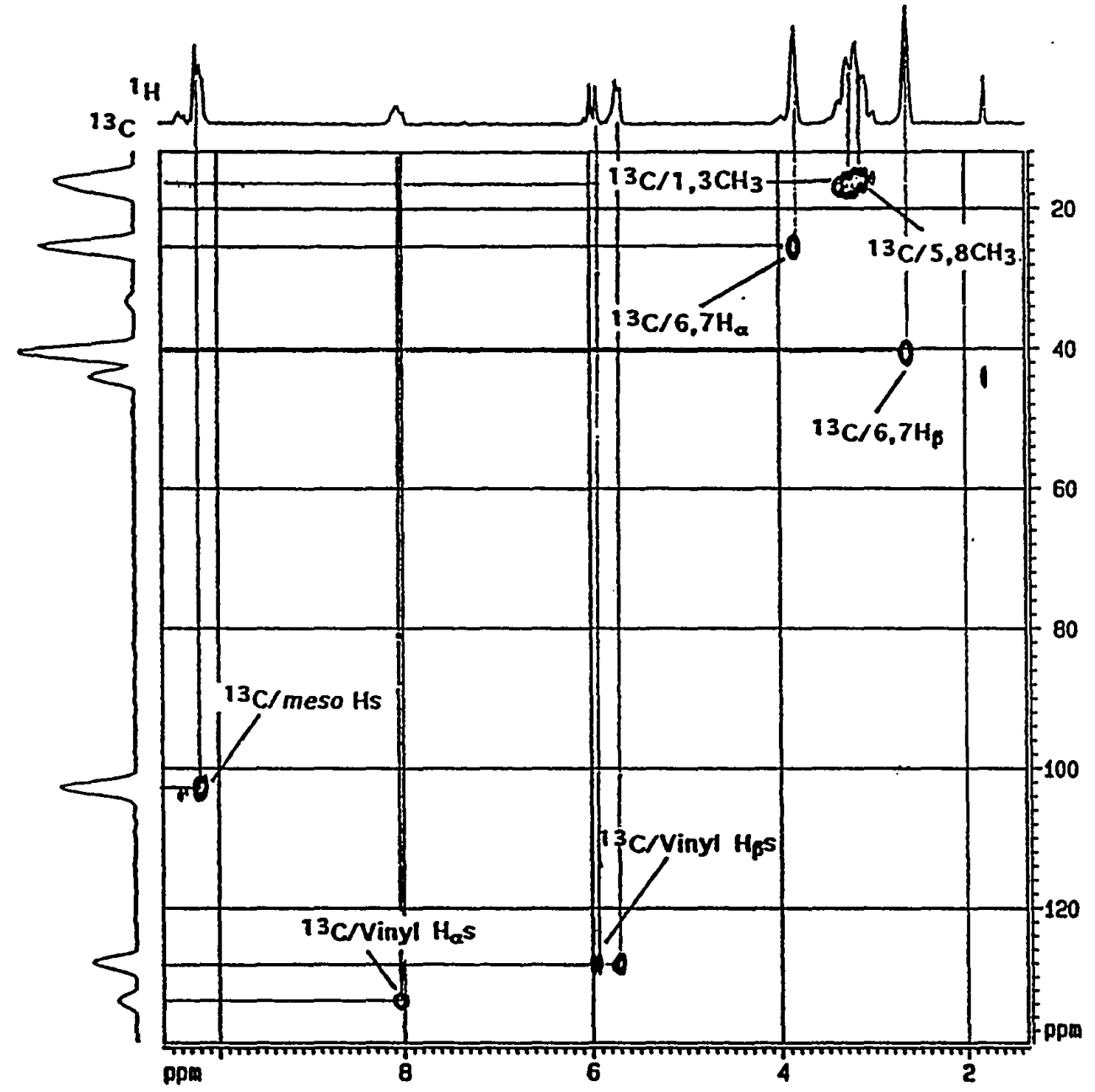

Eigure $10 .{ }^{13} \mathrm{C}:{ }^{1} \mathrm{H}-$ Inverse detection spectroscopy. Two-dimensional inverse detection spectroscopy with scalar coupling between the protons and carbons of the porphyrin ring 

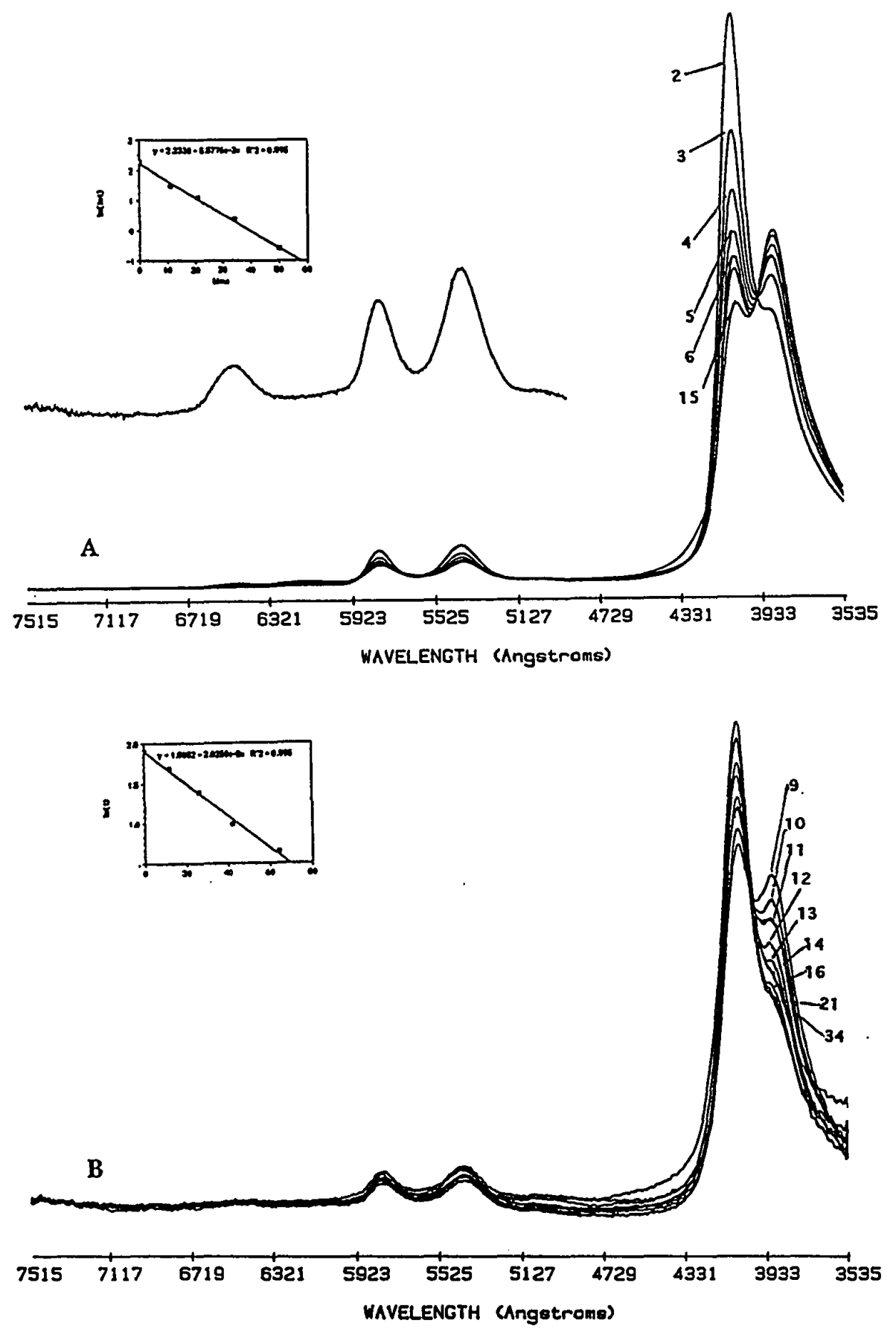

Eigure 11. Optical spectra at $25^{\circ} \mathrm{C}$. (A) Dimer formation showing a drop in intensity at $405.8 \mathrm{~nm}$. (B) Dimer dissociation upon dilution with a gain in intensity a $405.8 \mathrm{~nm}$. 


\section{CHAPTER V}

\section{${ }^{1} \mathrm{H}$ AND ${ }^{119}$ SN MAGNETIC RESONANCE STUDY OF THE PORPHYRIN-BINDING POCKET OF THE EQUILIBRIUM STRUCTURE OF TIN (IV) PROTOPORPHYRIN IX COMPLEX \\ OF EQUINE MYOGLOBIN}

The interactions of porphyrins with proteins are important from a number of perspectives. Heme, and presumably SnPP, are transported in serum as the complexes of the proteins hemopexin and serum albumin (Muller-Eberhard, 1988; Breslow et al., 1986). Although serum albumin and hemopexin are intrinsically interesting as SnPP transport molecules, the large size and relative paucity of solution state structural information for serum albumin and hemopexin would make exhaustive sequence-specific ${ }_{1}^{1} \mathrm{H}$ NMR assignments impossible. Therefore, investigation of the binding of SnPP to $\mathrm{Mb}$ as a starting point is useful because the interaction of heme with $\mathrm{Mb}$ is well characterized. Furthermore, the magnetic properties of ${ }^{119} \mathrm{Sn}$ makes it possible to probe the structure of $\mathrm{SnPP} \cdot \mathrm{EqMb}$ from the perspective of the center of the porphyrin moiety.

This chapter presents detailed ${ }^{1} \mathrm{H}$ NMR evidence for the solution state structure of the heme-binding pocket of the complex of SnPP with EqMb. The X-ray crystal structures for SwMbs (Takano, 1977a, 1977b) and EqMb (Evans and Brayer, 1988) facilitate this work greatly, as do the ${ }^{1} \mathrm{H}$ NMR assignments for the diamagnetic molecule SwMbCO (Mabbutt and Wright, 1985; Dalvit and Wright, 1987) and EqMbCO (Lecomte and Cocco, 1990). Throughout this chapter, references to these complexes will be made. The material presented in this chapter was included in the publication: Deeb, R.S., and Peyton, D.H. (1991) "1 H and ${ }^{119}$ Sn Magnetic Resonance Study of the 
$\mathrm{Sn}^{\mathrm{IV}}$ Protoporphyrin IX Complex of Equine Myoglobin: Structure of The Porphyrin-Binding Pocket" J. Biol. Chem. 266, 3728-3733.

\section{MATERIALS AND METHODS}

SnPP was purchased from Porphyrin Products. SnPP solutions were always prepared fresh by dissolving the porphyrin in a minimum amount of base $\left(\mathrm{NaO}^{2} \mathrm{H}\right.$ in $\left.{ }^{2} \mathrm{H}_{2} \mathrm{O}\right)$ or ( $\mathrm{NaOH}$ in ${ }^{1} \mathrm{H}_{2} \mathrm{O}$ ) solvent as required. ApoMb (preparation described in Chapter III) solutions were prepared fresh by dissolving the desired amount of protein in $50 \mathrm{mM}$ phosphate/200 mM NaCl buffer at $\mathrm{pH} 7.0$, or $0.1 \mathrm{M}$ borate $/ 200 \mathrm{mM} \mathrm{NaCl}$ buffer at $\mathrm{pH}$ 9.0. The amount of SnPP to add to apoMb was determined optically; titrations were performed by measuring optical differences at $418 \mathrm{~nm}$ employing the Cary 16 spectrophotometer described in Chapter II. After forming the 1:1 complex of SnPP.EqMb, the solution was exchanged into a deuterated phosphate buffer or a deuterated borate buffer, depending on the required $\mathrm{pH}$, and then concentrated by ultrafiltration over an Amicon YM-5 membrane to $3 \mathrm{mM}$ at $0.5 \mathrm{~mL}$.

Although unstable to lyophilizing, SnPP•EqMb solutions in buffer last for several weeks at room temperature if kept in the dark. Exposure to ambient light, however, causes rapid and irreversible precipitation of the complex. Direct evidence for stability of $\mathrm{SnPP} \cdot \mathrm{EqMb}$ is found in the reluctance of many amide protons to exchange in ${ }^{2} \mathrm{H}_{2} \mathrm{O}$ (see below).

CarbonmonoxyEqMb (EqMbCO) was prepared by dissolving the desired amount of EqMb in deuterated $50 \mathrm{mM}$ phosphate $200 \mathrm{mM} \mathrm{NaCl}$ at $\mathrm{pH}$ 7.0. The buffer was first purged with $\mathrm{N}_{2}$ then $\mathrm{CO}$. Mb was reduced (Mabbutt and Wright, 1985) with sodium dithionite, forming a cherry-red solution, and excess dithionite was removed either by ultrafiltration over a YM-5 Amicon membrane or by Sephadex G-25 gel filtration in the 
buffer saturated with $\mathrm{CO}$. The results from the EqMbCO NMR spectra verified Lecomte and Cocco's EqMbCO assignments (Lecomte and Cocco, 1990), and served as a very good visual comparison to our SnPP•EqMb work.

\section{RESULTS}

EqMb and SwMb have a high sequence homology (Dayhoff, 1972) and practically identical tertiary structures (Evans and Brayer, 1988). Due to the protection of sperm whales, and therefore the limited sources of SwMb, EqMb was a good alternate choice. The extant SwMb data aided greatly in the assignment process of SnPP.EqMb, because assignment strategy was modeled directly after that applied to SwMbCO. The resonance assignment strategy used is first to assign the porphyrin resonances as far as possible, then use NOEs to assign resonances from amino acid residues, based on their proximity to the porphyrin. Figure $12 \mathrm{~A}$ and $\mathrm{B}$ show the major and minor porphyrin-insertion isomers which have been found for native $\mathrm{Mb}$ ( $\mathrm{La}$ Mar et al, 1983). Figure $12 \mathrm{C}$ and $\mathrm{D}$ show the positions of various proximal and distal residue side chains relative to the major porphyrin-insertion isomer.

\section{The 1 H NMR Spectrum of SnPP.EQMb}

The chemical shifts for amino acids follow a general trend. Amide protons are generally the most downfield, in a region between 7 and $9 \mathrm{ppm}$. Aromatic protons generally fall between 6 and $7.5 \mathrm{ppm}$, followed by aliphatic protons which appear anywhere between 1 and $5 \mathrm{ppm}$. It is important to note however that in proteins, the location of amino acids with respect to each other can cause enormous shifts in the resonance positions when compared to resonance positions in the free amino acids. This information is relevant to the secondary and tertiary structure of the protein. A large 
number of NOEs are always observed in globular proteins, reflecting the fact that these molecular structures contain numerous pairs of closely spaced hydrogen atoms. Combined with resonance assignments, these proximities can provide secondary structure information. With model building, spatial arrangements in principle can be generated for the polypeptide chain that reflect their distance constraints.

Tracings of the downfield and upfield ${ }^{1} \mathrm{H}$ NMR spectral regions for SnPP, SnPP-EqMb, EqMbCO, and apoEqMb are shown in Figure $13 \mathrm{~A}-\mathrm{D}$. SnPP•EqMb has four downfield resonances that are from the four meso protons (the $\delta$ and $\alpha$ meso protons overlap at $\sim 10.7 \mathrm{ppm}$ at $\mathrm{pH} 7$ ) between 10 and $11.5 \mathrm{ppm}$, and a well-resolved upfield resonance at $-2.72 \mathrm{ppm}$, which is strongly analogous to one of the $\mathrm{Val}(68) \mathrm{E} 11 \mathrm{XCH}_{3}$ resonances in SwMbCO (Dalvit and Wright 1987; Mabbutt and Wright, 1985), EqMbCO (Figure 13 C, and Lecomte and Cocco, 1990), or desFeSwMb (La Mar et al., 1989). There is extensive dispersion of chemical shifts of the remaining envelope of resonances, indicating strongly ordered secondary and tertiary structures (Wüthrich, 1986). It is therefore concluded that SnPP inserts into the globin in a way similar to one of the two heme-insertion isomers found in the native $\mathrm{MbCO}$. Moreover, the apoEqMb spectrum of Figure $13 \mathrm{D}$ presents evidence of global folding as seen from several resonances in chemical shift positions not indicative of a 'random coil', especially a number of resonances upfield of $0.5 \mathrm{ppm}$. This conclusion corroborates $\mathrm{CD}$ experiments of apoMb (Harrison and Blout, 1965), and calorimetry of apoMb (Griko et al., 1988). A similar observation has been reported for apocytochrome b5 (Moore and Lecomte, 1990). Questions of more subtle structural interactions in SnPP.EqMb remain, such as conformations of the porphyrin-contact residues, the orientation of SnPP relative to the protein matrix, and the coordination pattern of the central metal ion. These issues will be addressed both here and in Chapter VI. Only one porphyrin-insertion isomer is detected at equilibrium (Figure $13 \mathrm{~B}$ ). Several exchangeable protons are present in the $7-9 \mathrm{ppm}$ 
region of Figure 13 B. However, if the reconstitution is carried out by first dissolving the apoMb in ${ }^{2} \mathrm{H}_{2} \mathrm{O}$ solvent, and then adding SnPP in ${ }^{2} \mathrm{H}_{2} \mathrm{O}$, this region exhibits fewer resonances, as a result of ${ }^{2} \mathrm{H}$-for-1 $1 \mathrm{H}$ exchange in the more 'open', solvent-accessible structure of apoMb. The averaged meso proton chemical shift of SnPP and SnPP.EqMb (Figure $13 \mathrm{~A}$ and $13 \mathrm{~B}$ ) is significantly greater than that of EqMbCO (Figure $13 \mathrm{C}$ ). The averaged meso proton chemical shift of desFeSwMb (La Mar et al., 1989) is intermediate between SnPP•EqMb and EqMbCO.

\section{Assignment of Porphyrin Resonances}

Figure 14 shows NOESY/DQF-COSY spectral regions of SnPP•EqMb at pH 9.0 which permit complete porphyrin resonance assignments, except for the 6- and 7propionate protons. Starting from the meso $\mathrm{H}_{\gamma}$, the resonance assignments for $\mathrm{SnPP}$ in the $\mathrm{Mb}$ pocket can be assigned from connectivities to neighboring groups on the prosthetic ring. The absence of any NOE connectivities to methyl or vinyl groups from the resonance at $11.30 \mathrm{ppm}$ identifies this resonance as the meso $\mathrm{H}_{\gamma}$. Two broad NOEs at 5.25 and $4.58 \mathrm{ppm}$ appear from the meso $\mathrm{H}_{\gamma}$. These resonances were not further assigned due to their location in a crowded region, next to the water resonance, but their likely origin as from the porphyrin propionates is noted. Each of the remaining meso resonances must show an NOE to at least one porphyrin methyl. At $\mathrm{pH} 7$ the meso $\mathrm{H}_{\delta}$ and meso $\mathrm{H}_{\alpha}$ are not resolved, however, at $\mathrm{pH} 9$ they are resolved from one another, and located at 10.70 and $10.72 \mathrm{ppm}$. The meso $\mathrm{H}_{\delta}$ shows NOE connectivities to two methyl resonances, which must then be the $1 \mathrm{CH}_{3}$ and $8 \mathrm{CH}_{3}$, at 3.90 and $3.98 \mathrm{ppm}$. The 3.98 ppm methyl shows an NOE to a vinyl $\mathrm{H}_{\beta \mathrm{t}}$ at $6.12 \mathrm{ppm}$, and so is the $1 \mathrm{CH}_{3}$ with the adjacent $2 \mathrm{H}_{\beta \mathrm{t}}$. The $3.90 \mathrm{ppm}$ methyl has no NOE to any vinyl proton, so is the $8 \mathrm{CH}_{3}$. The COSY spectrum shows the expected scalar connectivities of $2 \mathrm{H}_{\beta \mathrm{t}}$ to $2 \mathrm{H}_{\alpha}(8.63 \mathrm{ppm})$ and $2 \mathrm{H}_{\alpha}$ to $2 \mathrm{H}_{\beta c}$ (6.18 ppm). 
The $2 \mathrm{H}_{\alpha}$ shows an NOE to the meso $\mathrm{H}_{\alpha}$ proton at $10.72 \mathrm{ppm}$, which itself shows an NOE to the $3 \mathrm{CH}_{3}$ at $4.02 \mathrm{ppm}$. The $3 \mathrm{CH}_{3}$ shows an $\mathrm{NOE}$ to $4 \mathrm{H}_{\beta \mathrm{t}}$ at $6.96 \mathrm{ppm}$, which, in turn, shows COSY connectivity to the $4 \mathrm{H}_{\alpha}$ at $8.79 \mathrm{ppm}$. COSY also identifies the $4 \mathrm{H}_{\beta \mathrm{c}}$, at $6.86 \mathrm{ppm}$, from its connectivity to $4 \mathrm{H}_{\alpha}$. The methyl-to-vinyl NOE intensities indicate that both vinyl groups are nearly in plane with the porphyrin in the so-called 'cis' orientation (drawn in Figure 12) with the $\mathrm{H}_{\alpha}$ s oriented toward the nearest meso, and not the nearest methyl.

A strong NOE exists between the $4 \mathrm{H}_{\alpha}$ and the meso $\mathrm{H}_{\beta}(10.14 \mathrm{ppm})$; the meso $\mathrm{H}_{\beta}$ shows a strong $\mathrm{NOE}$ to the $5 \mathrm{CH}_{3}$ at $2.84 \mathrm{ppm}$. The $5 \mathrm{CH}_{3}$ is shifted to its position, greater than $1 \mathrm{ppm}$ upfield of the other porphyrin methyls, because of the ring current effects from nearby aromatic amino acid residues (Figure $12 \mathrm{D}$ ). This $5 \mathrm{CH}_{3}$ chemical shift is similar in SwMbCO and EqMbCO, and so the only SnPP insertion isomer observed in $\mathrm{SnPP} \cdot \mathrm{EqMb}$ at equilibrium is as illustrated in Figure $12 \mathrm{~A}, \mathrm{C}$ and $\mathrm{D}$. Based on these assignments and conclusions, the porphyrin protons provide probes from which the environment of the prosthetic group can be explored.

The residue assignment strategy starts by using COSY/two-quantum spectroscopy to determine spin systems, and then use NOEs to the porphyrin and other residues to piece together the 3-dimensional puzzle.

\section{Phe(43)CD1 (Distal)}

DQF-COSY and TOCSY spectra show a well resolved phenylalanine ring pattern with resonances at 7.12,5.52, and 3.48. This pattern is characteristic of the phenyl ring spin system $A^{\prime} A^{\prime} B B^{\prime} M$, such that $A$ and $A^{\prime}$ represent the $\delta$ protons, $B$ and $B^{\prime}$ represent the $\varepsilon$ protons, and $M$ represents the $\zeta$ proton. The $\delta$ Hs at $7.12 \mathrm{ppm}$ show NOESY, COSY, and TOCSY correlation to the $\varepsilon H s$ at $5.52 \mathrm{ppm}$, which in turn show connectivity to the 
$\zeta \mathrm{H}$ at $3.48 \mathrm{ppm}$. TOCSY corroborates this assignment by showing scalar connectivity between the $\zeta \mathrm{H}$ and the $\delta \mathrm{Hs}$ (Figure 15).

Two-quantum spectroscopy was another NMR method used to obtain subspectra containing exclusively resonances from spin systems located in a crowded region. Figure 16 clearly shows the ring protons of this phenylalanine at the chemical shifts discussed above.

Based on the location of the Phe(43)CD1 from previous NMR and X-ray studies on SwMbCO (Mabbutt and Wright, 1985; Dalvit and Wright, 1987;,Takano, 1977a, 1977b), Phe(43)CD1 was sought on the distal side of the porphyrin ring in close proximity to the meso $\mathrm{H}_{\beta}, 5 \mathrm{CH}_{3}$, and the $4 \mathrm{H}_{\alpha}$. Thus, these should show NOE connectivities to $5.52 \mathrm{ppm}$ and the $5 \mathrm{CH}_{3}$ at $2.84 \mathrm{ppm}$ (Figure 14).

Phe(43)CD1 can be distinguished from nearby phenylalanine residues by its proximity to the porphyrin $5 \mathrm{CH}_{3}, 4 \mathrm{H}_{\alpha}$ and meso $\mathrm{H}_{\beta}$ proton. Furthermore, NOE connectivities are observed from $\mathrm{Phe}(43) \mathrm{CD} 1 \varepsilon \mathrm{H}$ to $\mathrm{Phe}(33) \mathrm{B} 14$ عH at $5.52 \mathrm{ppm}$ (assigned below), and between Phe(43)CD1 $\delta \mathrm{H}$ and Phe(33)B14 לु at $5.20 \mathrm{ppm}$. NOEs are observed between Phe(43)CD1 єH and His(64)E7 عH (also assigned below) at 5.18 ppm (Figure 15). The resonances for this residue are significantly upfield-shifted relative to SwMbCO, elephant $\mathrm{MbCO}$ or EqMbCO. Although it is very unusual to have an aromatic proton at $3.48 \mathrm{ppm}$, the results of this assignment confirm its location within the protein matrix. It is possible that Phe(43)CD1 might be slightly shifted relative to these other species in its location above the edge of the porphyrin ring; this can be sufficient to cause significant differences in chemical shifts due to ring current effects (Johnson and Bovey, 1958). 
Phe(33)B14 (Distal)

The assignment strategy for the Phe(33)B14 aromatic spin system was obtained in the same way Phe(43)CD1 was determined. Figure 15 shows NOESY, COSY, and TOCSY connectivities for the aromatic protons. The $\delta \mathrm{Hs}$ are observed at $6.96 \mathrm{ppm}$ which show connectivities to the $\varepsilon \mathrm{Hs}$ at $6.37 \mathrm{ppm}$, which in turn show correlation to the $\zeta \mathrm{H}$ at 5.20 ppm. Two-quantum spectroscopy confirms the assignments for this spin system in a similar way to Phe(43)CD1 seen in Figure 16.

Phe(33)B14 can be distinguished from the other phenylalanine residues by NOEs to neighbors. This residue is near Leu(29)B10 (assigned below) and Phe(43)CD1, while Phe(46)CD4 is near Phe(43)CD1 and His(64)E7. The NOE connectivities from Phe(33)B14 to Phe(43)CD1 are discussed in the Phe(43)CD1 section. An NOE between Phe(33)B 14 לH and Leu(29)B10 $8 \mathrm{H}$ is observed at $5.20 \mathrm{ppm}$ (Figure 17). Leu(29)B10 will be discussed in more detail below. The chemical shifts for the protons in this residue are in almost the same location as they are in EqMbCO and SwMbCO, indicating that the location of Phe(33)B14 in the heme pocket of SnPP.EqMb is close to its location in these species.

\section{Phe(46)CD4 (Distal)}

This residue is distinguished from the other phenylalanine residues by its exclusive proximity to His(48)CD6 as seen from SwMbCO (Dalvit and wright, 1987). Phe(46)CD4 appears in a crowded region where cross peaks are very close to the diagonal (Figure 15). Therefore, it was not possible to assign the complete spin system. Tentative assignments of the $\delta \mathrm{Hs}$ at $6.55 \mathrm{ppm}$ and the $\varepsilon \mathrm{H}$ at $6.31 \mathrm{ppm}$ were made based on the following NOESY connectivities. A strong NOE from $\delta \mathrm{H}$ of Phe(46)CD4 to the $\varepsilon H$ of His(64)E7 at 7.97 ppm was observed, as well as an NOE from the $\varepsilon H$ of Phe(46)CD4 to the $\varepsilon \mathrm{H}$ of Phe(43)CD1 at $5.52 \mathrm{ppm}$ (Figure 15). 
$\operatorname{Val}(68) \mathrm{E} 11$ (Distal)

NOEs are also observed from the porphyrin $1 \mathrm{CH}_{3}$ to $\mathrm{Val}(68) \mathrm{E} 11 \mathrm{\gamma CH}_{3}$ at $3.98 \mathrm{ppm}$ (Figure 17). In the aliphatic region (Figure 17), the most upfield shifted signal resonates at $-2.72 \mathrm{ppm}$ and has COSY and TOCSY patterns indicating a valine methyl group with a companion methyl at $-0.49 \mathrm{ppm}$. The valine residue side chain $\mathrm{CH}_{\beta}\left(\mathrm{CH}_{3 \gamma}\right)_{2}$ is expected to show scalar connectivities as well as NOE connectivities between the $\beta \mathrm{CH}$ and the two $\mathrm{\gamma CH}_{3} \mathrm{~S}$.

Figure 17 shows COSY and NOESY cross peaks between Val(68)E11 $\gamma_{\mathrm{CH}} \mathrm{S}$ at -0.49 ppm and another between $\mathrm{Val}(68) \mathrm{E} 11 \beta \mathrm{CH}$ and $\mathrm{\gamma CH}_{3}$ at $0.82 \mathrm{ppm}$. A TOCSY and NOESY connectivity was observed between the $\alpha \mathrm{CH}$ of $\mathrm{Val}(68) \mathrm{E} 11$ and $\mathrm{CH}_{3}$ at 2.74 ppm to corroborate this assignment (not shown).NOEs are observed from the resonances of the porphyrin meso $\mathrm{H}_{\delta}$ to the $\alpha \mathrm{H}$ of $\mathrm{Val}(68) \mathrm{E} 11$ at $2.74 \mathrm{ppm}$ (Figure 14)

From the NMR and X-ray crystallographic data on SwMbCO, Val(68)E11 is in proximity to Leu(29)B10 and His(64)E7 on the distal side of the porphyrin ring. The NOESY spectrum of SnPP•EqMb (Figure 17) shows NOE connectivities between His(64)E7 $\varepsilon \mathrm{H}$ and $\mathrm{\gamma CH}_{3}$ of $\mathrm{Val}(68) \mathrm{E} 11$ at $5.18 \mathrm{ppm}$, as well as $\delta \mathrm{CH}_{3} \mathrm{~S}$ of Leu(29)B10 and $\mathrm{XCH}_{3}$ of $\mathrm{Val}(68) \mathrm{E} 11$ at -0.28 and $-0.73 \mathrm{ppm}$.

The $\mathrm{Val}(68) \mathrm{E} 11 \mathrm{CH}_{3}$ at $-2.72 \mathrm{ppm}$ is shifted slightly upfield relative to its position in EqMbCO and SwMbCO. This can possibly reflect a slight conformational difference between SnPP•EqMb and the two complexes. The valine residue would then be situated more above the porphyrin ring in SnPP.EqMb.

\section{Leu(29)B10 (Distal)}

The resonances of $\mathrm{Leu}(29) \mathrm{B} 10, \mathrm{CH}_{2 \beta} \mathrm{CH}_{\gamma}\left(\mathrm{CH}_{38}\right)_{2}$ are assigned analogously to SwMbCO. NOE connectivities between the two $8 \mathrm{CH}_{3} \mathrm{~s}$ at -0.82 and $-0.73 \mathrm{ppm}$ as well as between $\delta \mathrm{CH}_{3}$ s and $\gamma \mathrm{CH}$ at $0.89 \mathrm{ppm}$ are observed in Figure 17. 
A DQF-COSY spectrum confirms this assignment by revealing the scalar connectivities between $\delta \mathrm{CH}_{3}$ and the $\gamma \mathrm{CH}$ protons and between $\gamma \mathrm{CH}$ and $\mathrm{\beta CH}_{2}$ s (Figure 17). TOCSY cross peaks between $\mathrm{Leu}(29) \mathrm{B} 10 \gamma \mathrm{CH}$ and $\mathrm{BCH}_{2} \mathrm{~s}$ are missing due to rotating frame NOE cancellation, i.e., the positive ROESY peak is opposite in sign from the TOCSY peak, and cancellation of cross peaks can occur. This behavior is also noted for EqMbCO under the conditions these spectra were acquired.

Leu(29)B10 shows dipolar contacts with three residues close to the prosthetic group, Val(69)E11, His(64)E7 and Phe(33)B14. NOEs are observed to Phe(33)B14 $\zeta \mathrm{H}$ from the $\mathrm{SCH}_{3}$ of $\mathrm{Leu}(29) \mathrm{B} 10$ at $5.20 \mathrm{ppm}$ and from His(64)E7 $\mathrm{EH}$ to the $\gamma \mathrm{CH}$ of $\mathrm{Leu}(29) \mathrm{B} 10$ at $5.18 \mathrm{ppm}$. NOEs are also observed between $\gamma_{\mathrm{CH}}$ of $\mathrm{Val}(68) \mathrm{E} 11$ and the $8 \mathrm{CH}_{3} \mathrm{~s}$ of Leu(29)B10 at -0.28 and $-0.73 \mathrm{ppm}$ (Figure 17).

This residue seems to be in approximately the same location with respect to the prosthetic group when compared to SwMbCO and EqMbCO. This is judged from the very similar chemical shifts of SnPP•EqMb when compared to the chemical shifts of the other complexes and from the identities and magnitudes of the NOEs.

\section{His(64)E7 (Distal)}

The resonance of His(64)E7 is assigned analogous to SwMbCO. A strong NOE is observed between the prosthetic mesoH $\mathrm{H}_{\gamma}$ proton and $\mathrm{\varepsilon H}$ of $\mathrm{His}(64) \mathrm{E} 7$ at $5.18 \mathrm{ppm}$. This assignment is corroborated by the NOE connectivities to Val(68)E11 $\mathrm{YCH}$, Leu(29)B10 $\gamma \mathrm{CH}$, Phe(43)CD1 EH, and to Phe(46)CD4 $8 \mathrm{H}$ at $5.18 \mathrm{ppm}$.

The resonances from this residue are strongly shifted upfield relative to its position in the SwMbCO spectrum. The $\delta \mathrm{H}$ seems to be buried under the water signal in SnPP.EqMb. These shifts indicate that HisE7 may be situated closer to the center of the prosthetic group than SwMbCO. 
Ile(99)FG5 (Proximal)

All strong NOEs to the porphyrin meso $\mathrm{H}_{\beta}$ proton have been assigned except for one at $1.13 \mathrm{ppm}$ (Figure 14); this proton also has an NOE to the $4 \mathrm{H}_{\alpha}$. The $1.13 \mathrm{ppm}$ peak is tentatively assigned to Ile(99)FG5 based on the location of the residue from the X-ray crystal structure of SwMbCO (Figure 12). This NOE however is significantly weaker in the EqMbCO spectrum obtained under similar conditions. The NOE intensity differences indicate subtle structural changes between EqMbCO and SnPP•EqMb.

\section{His(97)FG3 (Proximal)}

Two histidine residues are normally located at the proximal side of the heme in SwMbCO: His(93)F8 and His(97)FG3 which forms a salt bridge to the 7-propionate group. In SnPP•EqMb, the $\varepsilon \mathrm{H}$ of His(97)FG3 was found at $8.03 \mathrm{ppm}$ as opposed to 8.54 ppm in SwMbCO. It was not possible to determine the location of this residue with respect to the propionates in SnPP.EqMb because crowding in the spectral region in question. An NOE from the porphyrin mesoH $_{\gamma}$ proton to $8.03 \mathrm{ppm}$ is identified as arising from His(97)FG3 $\mathrm{\varepsilon H}$; as expected from the crystal structure, this proton does not show an NOE to any assigned distal residue protons. A summary of all resonance assignments is provided in Table III.

\section{The Proximal His-Fe Bond:119 Sn NMR}

The proximal histidine, His(93)F8 is covalently bound to iron atom in SwMbCO; this causes the $\delta \mathrm{H}$ and $\varepsilon \mathrm{H}$ resonances to be extremely upfield shifted to 1.13 and $1.66 \mathrm{ppm}$ in SwMbCO (Dalvit and Wright, 1987) due to ring current effects from the porphyrin ring. It was not possible to locate these protons from the NMR spectra of SnPP.EqMb. However, ${ }^{119} \mathrm{Sn}$ spectroscopy helped establish the existence of a bond between this residue and the porphyrin Sn as in His(93)F8 in SwMbCO. Figure 18 demonstrates the ${ }^{119} \mathrm{Sn}$ resonance of $\mathrm{SnPP} \cdot \mathrm{EqMb}$, both without and with WALTZ proton decoupling 
(during acquisition only). The ${ }^{119} \mathrm{Sn}$ signal is sharpened in the ${ }^{1} \mathrm{H}$-decoupled spectrum, and so scalar coupling (on the order of $5-15 \mathrm{~Hz}$ ) from proton(s) of the amino acid in question to the $\mathrm{Sn}$ have been removed. This implies that the coordination pattern probably includes at least one axial ligand to the $\mathrm{Sn}$. The coupling to protons cannot arise from coordinated water because the ${ }^{119} \mathrm{Sn}$ spectra were recorded in ${ }^{2} \mathrm{H}_{2} \mathrm{O}$ to remove the effects of $\mathrm{H}_{2} \mathrm{O}$ solvent. This coupling cannot arise from porphyrin peripheral protons because the ${ }^{119} \mathrm{Sn}$ NMR signal of SnPP is a single line of width $\sim 15 \mathrm{~Hz}$, and is not narrowed by ${ }^{1} \mathrm{H}$-decoupling (shown in Chapter IV, Figure 8).

\section{The Distal Fe-OH $(2): 1$ H NMR pH-Titration}

Distal His(64)E7 in SnPP.EqMb has a ring proton in nearly the same chemical shift position as in other diamagnetic globins. This distal ring cannot be coordinated to $\mathrm{Sn}$ because if it were, the histidine $\delta \mathrm{H}$ and $\mathrm{eH}$ would have had extreme upfield ring-current shifts from the porphyrin (Johnson and Bovey, 1958; Dalvit and Wright, 1987). During titration from pH 7 to 10 (Figure 19), all four heme mesoHs showed perturbed chemical shifts. The $\mathrm{pK}_{\mathrm{a}}$ affecting each meso proton chemical shift change was determined to be 9.8, and so the group being titrated is likely to be coordinated to water (titration of $\mathrm{H}_{2} \mathrm{O}$ to $\mathrm{OH}^{-}$) similarly as in metMbH $\mathrm{O}_{2} \mathrm{OH}^{-}$(McGrath and $\mathrm{La} \mathrm{Mar}$ 1978). Therefore, the fifth and sixth ligands to Sn appear to be the His(93)F8 imidazole on the proximal side of the porphyrin and water (or $\mathrm{OH}^{-}$at high $\mathrm{pH}$ ) ligand occupying the distal side.

\section{DISCUSSION}

The initial observations to be made from the ${ }^{1} \mathrm{H}$ NMR assignments of SnPP•EqMb are that a single SnPP insertion isomer is detected in SnPP.EqMb at equilibrium, and that the porphyrin is inserted into the globin in very much the same way as is heme in $\mathrm{MbCO}$. 
TABLE III

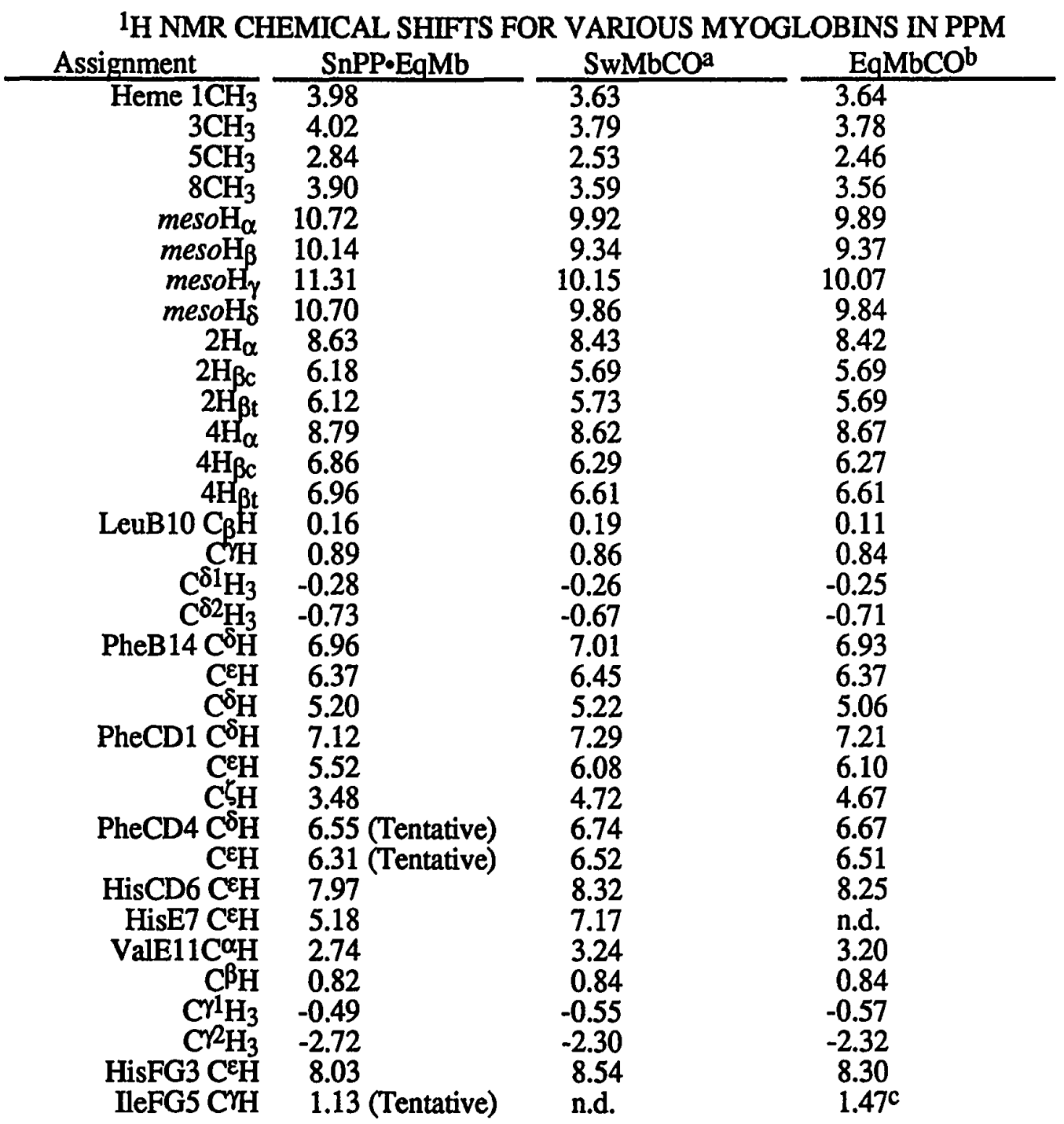

aTaken from Dalvit and Wright (1987). n.d., not determined. bTaken from Lecomte and Cocco (1990). cTaken from Emerson and La Mar (1990).

The nearly identical NOE patterns for SnPP.EqMb and native EqMbCO, both on the distal and the proximal side of the porphyrin, are striking, indicating that deformation of the binding pocket due to the Sn-for-Fe substitution must be minimal. Exceptions to this 
trend are distal His(64)E7 with its $\mathrm{EH}$ chemical shift considerably upfield of that found in EqMbCO, Phe(43)CD1, with its significant upfield shifts of its $\varepsilon H$ and $\zeta H$, and His(97)FG3 with its EH upfield of that found in SwMbCO. Because His(64)E7 is situated just above the edge of the porphyrin skeleton, even a small shift in the location of this proton relative to the porphyrin would cause a significant shift in its position due to ring current effects (Johnson and Bovey, 1958). The Val(68)E11 $\mathrm{gCH}_{3}$ at $-2.72 \mathrm{ppm}$ in SnPP.EqMb is $\sim 0.4 \mathrm{ppm}$ upfield of its position in EqMbCO, possibly reflecting a slight conformational difference in the two complexes. It appears that SnPP•EqMb has a sixth ligand (water), and so models metMbH $\mathrm{H}_{2} \mathrm{O}$. In metMbH${ }_{2} \mathrm{O}$, the His $\mathrm{E} 7$ side chain $\mathrm{\varepsilon N}$ is hydrogen-bonded to the water, but in deoxyMb the HisE7 side chain is further from the iron, even though the $\mathrm{E}$ helix is in closer proximity to the heme (Takano, 1977a,b). The chemical shifts for His(64)E7 and $\mathrm{Val}(68) \mathrm{E} 11$ reported here are consistent with the side chains from positions E7 and E11 being drawn toward the metal (relative to this position in $\mathrm{MbCO}$ ) by hydrogen bonding between HisE7 and the water ligand on the distal side of the porphyrin ring. SnPP•Mb may thus be a diamagnetic model for the six-coordinate high-spin paramagnetic molecule, metMbH $_{2} \mathrm{O}$ (Satterlee, 1986; La Mar, 1979) which is high spin and extremely difficult to study by NMR.

The use of ${ }^{119} \mathrm{Sn}$ NMR spectroscopy was important for assessing the proximal side of the porphyrin in SnPP•EqMb, particularly because it was not possible to assign the proximal histidine resonances by conventional two-dimensional ${ }^{1} \mathrm{H}$ NMR methods. It is unlikely that J-coupling between ${ }^{119} \mathrm{Sn}$ and ${ }^{117} \mathrm{Sn}$ (at $8.58 \%$ and $7.61 \%$ natural abundance, respectively) caused sufficient attenuation of the $\varepsilon H$ resonance intensity to completely obscure its NOE from the mesoH $\mathrm{H}_{\delta}$. The HisF8 $\mathrm{\varepsilon H}$ resonance is identified from its NOE to the mesoH $\delta$ in EqMbCO. This was not the case for SnPP.EqMb, indicating that the ring plane seems to be somewhat rotated (Traylor and Berzinis, 1980) relative to its position in $\mathrm{EqMbCO}$. The His(93)F8 ring amide proton, (N) $\delta \mathrm{H}$ is not 
possible to locate from SnPP-EqMb in ${ }^{1} \mathrm{H}_{2} \mathrm{O}$ solvent, using two-quantum spectroscopy. It is possible that this proton may be in sufficiently fast exchange to obscure its location (Han et al., 1989; Lecomte and La Mar, 1985; Cutnell et al., 1981; La Mar, 1979). However, the identity of this proton is also not obvious at $\mathrm{pH} 5.4$, at which the exchange rate is presumed to be slowed (Lecomte and Cocco, 1990). ${ }^{119} \mathrm{Sn}-{ }^{1} \mathrm{H}$ heteronuclear COSY (Bax, 1983) failed to provide chemical shifts for the His F8 ring protons, perhaps because the ${ }^{119} \mathrm{Sn}$ signal is broadened by interaction with the quadrupolar porphyrin nitrogens ( $S=1$, for $99.6 \%$ naturally abundant $15 \mathrm{~N}$ ). This is consistent with the $\sim 15 \mathrm{~Hz}$ linewidth of the ${ }^{119} \mathrm{Sn}$ signal from free SnPP (Chapter IV).

\section{ACKNOWLEDGEMENTS}

Thanks is given to Professor Juliette Lecomte (Pennsylvania State University) for enlightenment from her work with EqMbCO and apoEqMb previous to publication. 


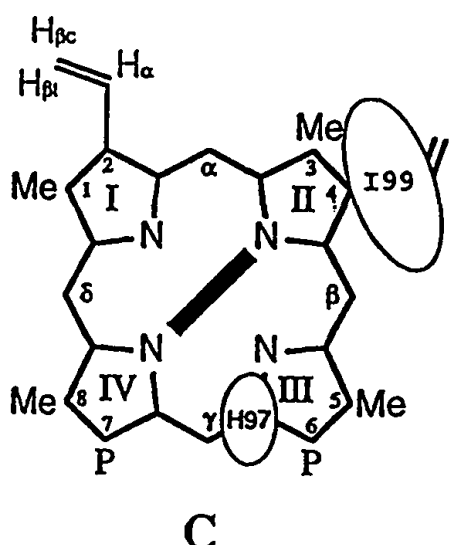

Proximal view

"Nomal" Insertion Isomer

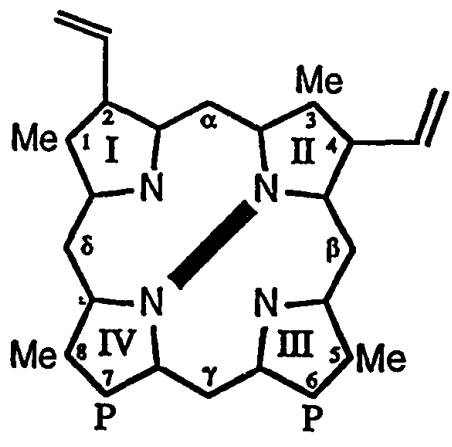

A

Proximal view

"Normal" Insertion Isomer

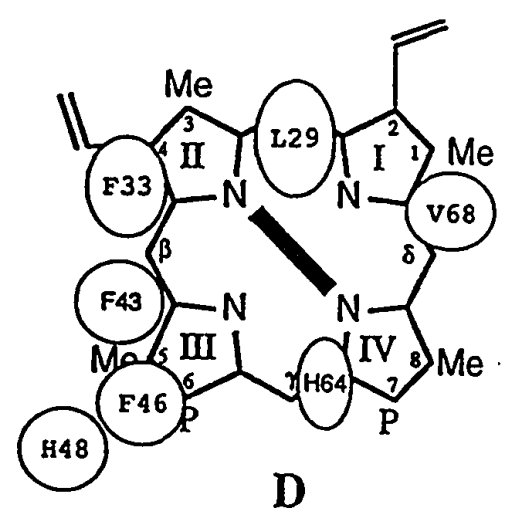

Distal view

"Normal" Insertion Isomer

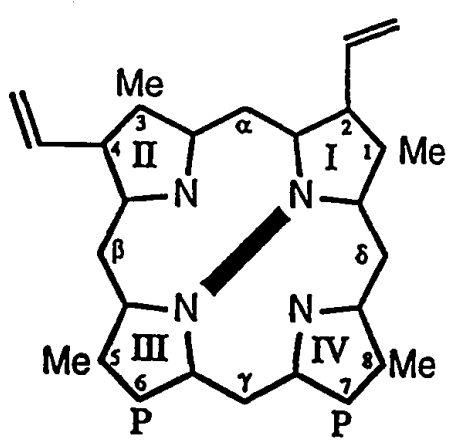

B

Proximal view

"Inverse" Insertion Isomer

Figure 12. Views of the porphyrin. (A) Proximal view of the porphyrin insertion isomer observed from the X-ray structures of EqMb and SwMb (La Mar et al., 1983).(B) Alternate or inverse heme insertion isomer, differing from that shown in $A$ by a $180^{\circ}$ "flip" of the porphyrin about the $\alpha-\gamma$ meso-axis. (C) Proximal view of the porphyrin insertion isomer observed from the $x$-ray structures of EqMb and $S w M b$, with two proximal residues included. (D) Distal view of the porphyrin insertion isomer observed from the $x$-ray structures of $\mathrm{EqMb}$ and $\mathrm{SwMb}$, with seven distal residues included. The bar in the middle of each diagram indicates the approximate position of the proximal histidine ring plane, and $\mathrm{P}$ means propionate. 

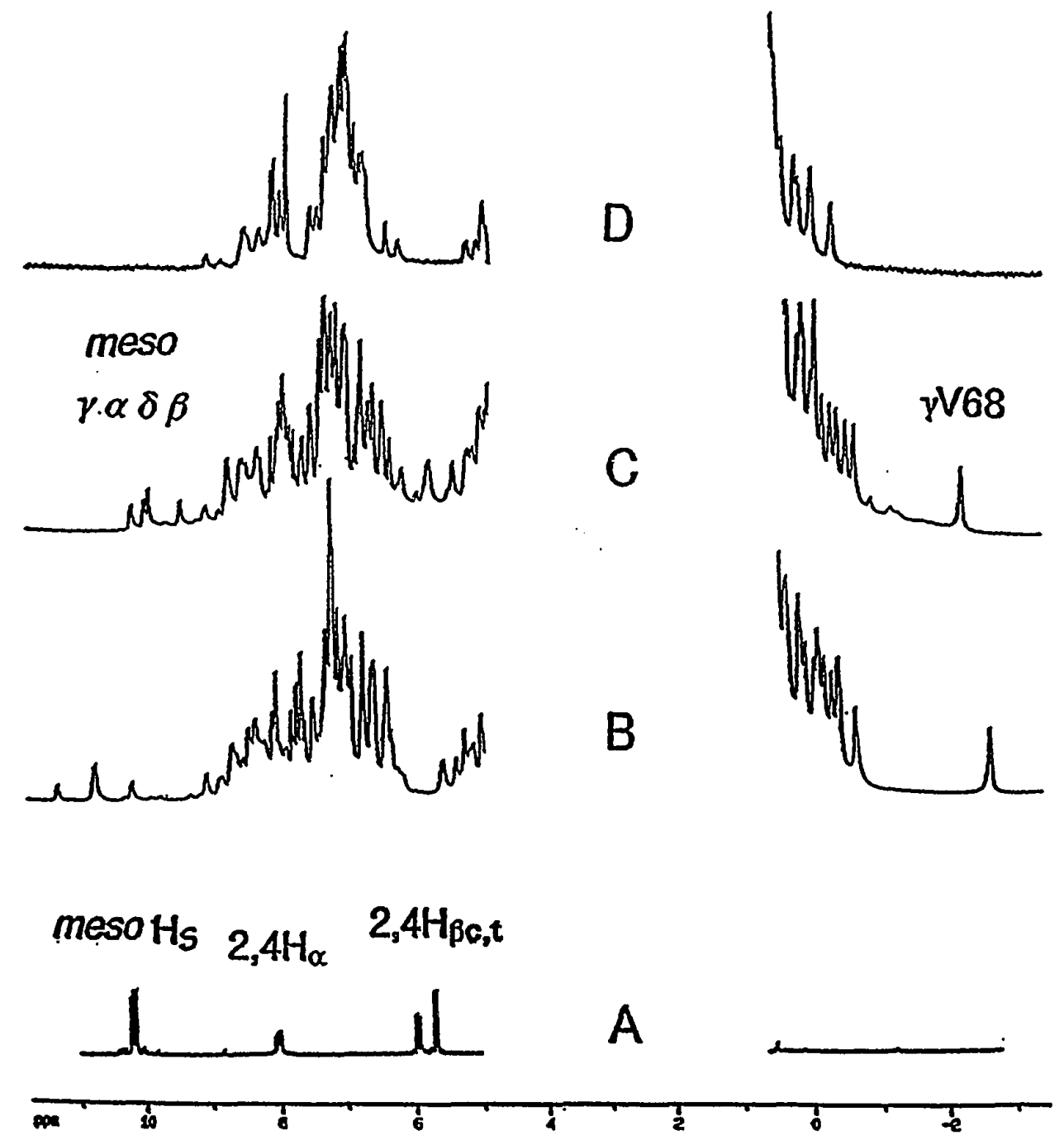

Eigure 13. One-dimensional NMR spectroscopy portions showing upfield and downfield regions. (A) SnPP in dimethyl sulfoxide-d6. (B) SnPP॰EqMb at $\mathrm{pH}$ 7.0. (C) EqMbCO at pH 7.0, with assignments of meso protons and one valine methyl. (D) ApoEqMb at pH 7.0. 


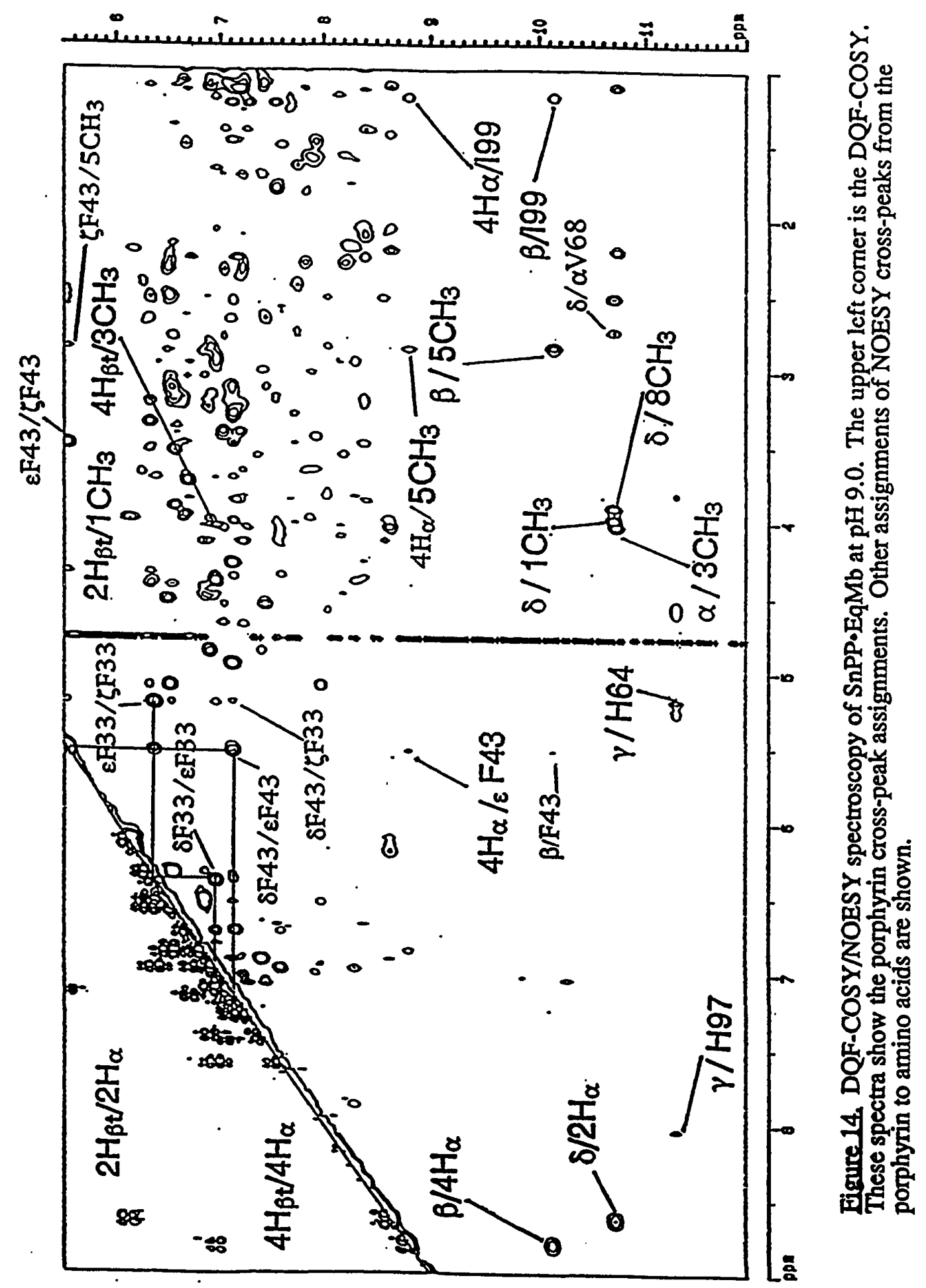



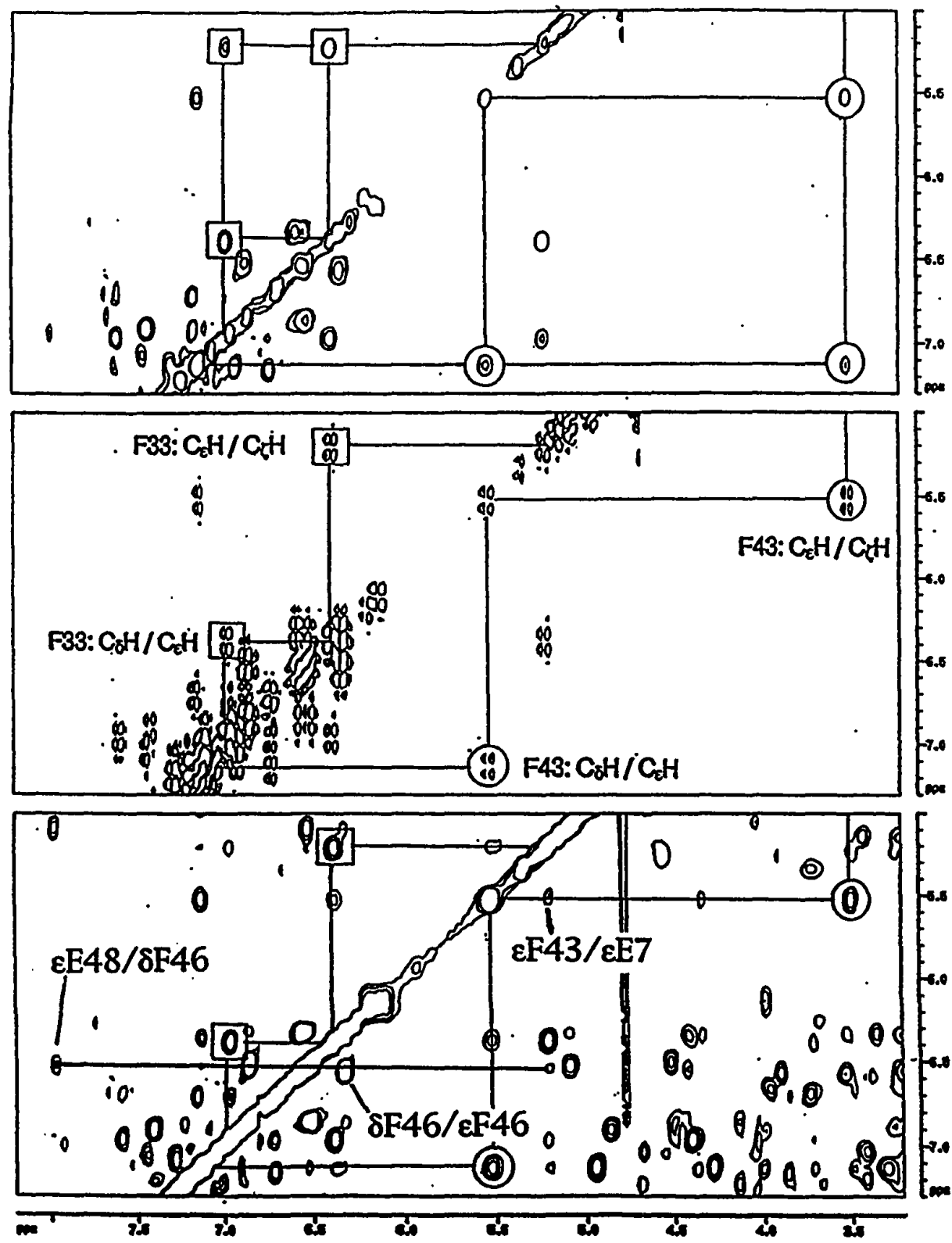

Eigure 15. Two-dimensional ${ }^{1} \mathrm{H}$ NMR spectra from the aromatic region of SnPP॰EqMb at pH 9.0. NOESY, COSY, TOCSY spectra, from the bottom. These spectra show the cross-peaks from the aromatic spin systems of Phe(33)B14 and Phe(43)CD1. 


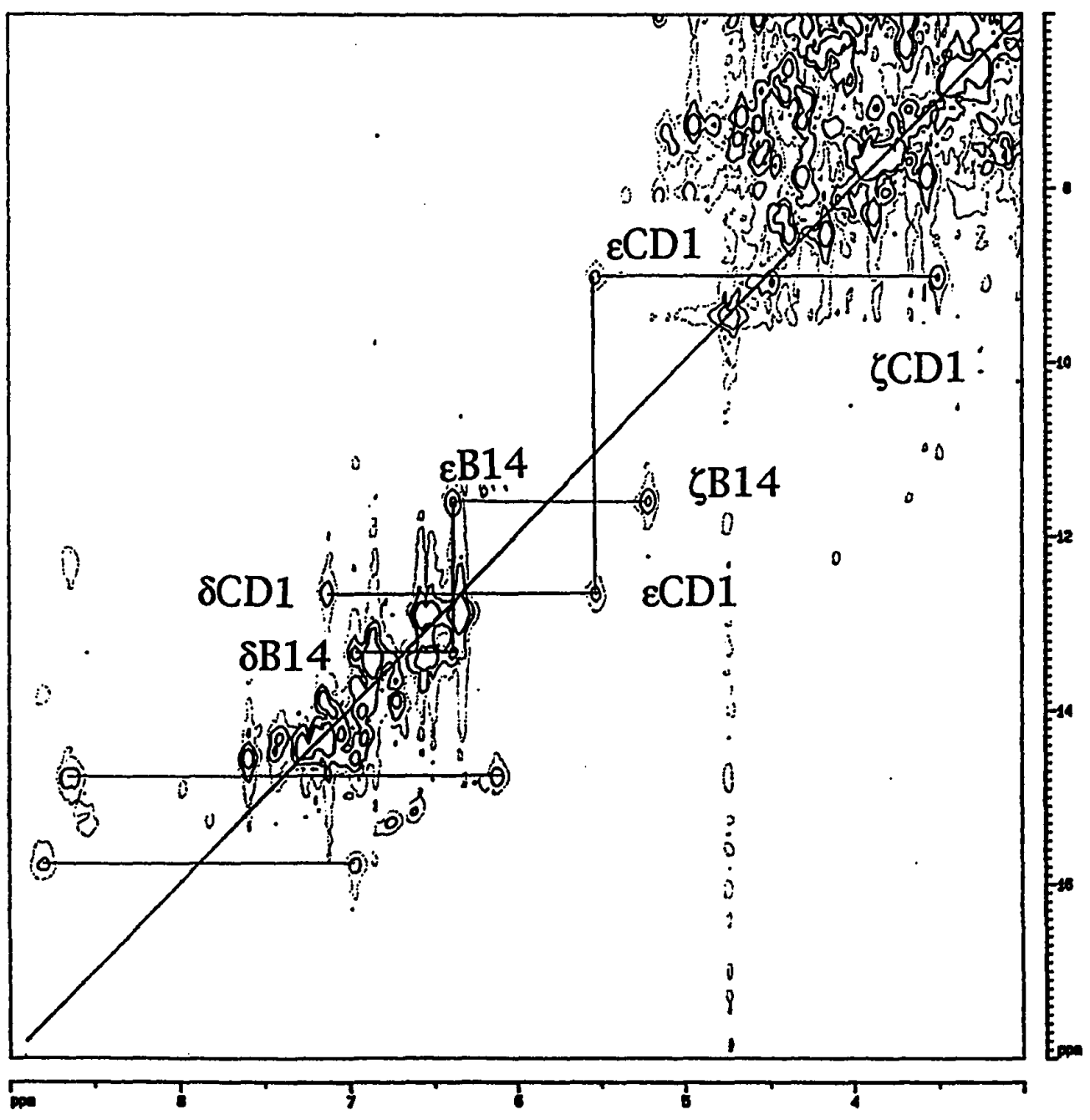

Eigure 16. Two-quantum ${ }^{1} \mathrm{H}$ NMR spectroscopy showing the Phe aromatic spin system connectivities. 


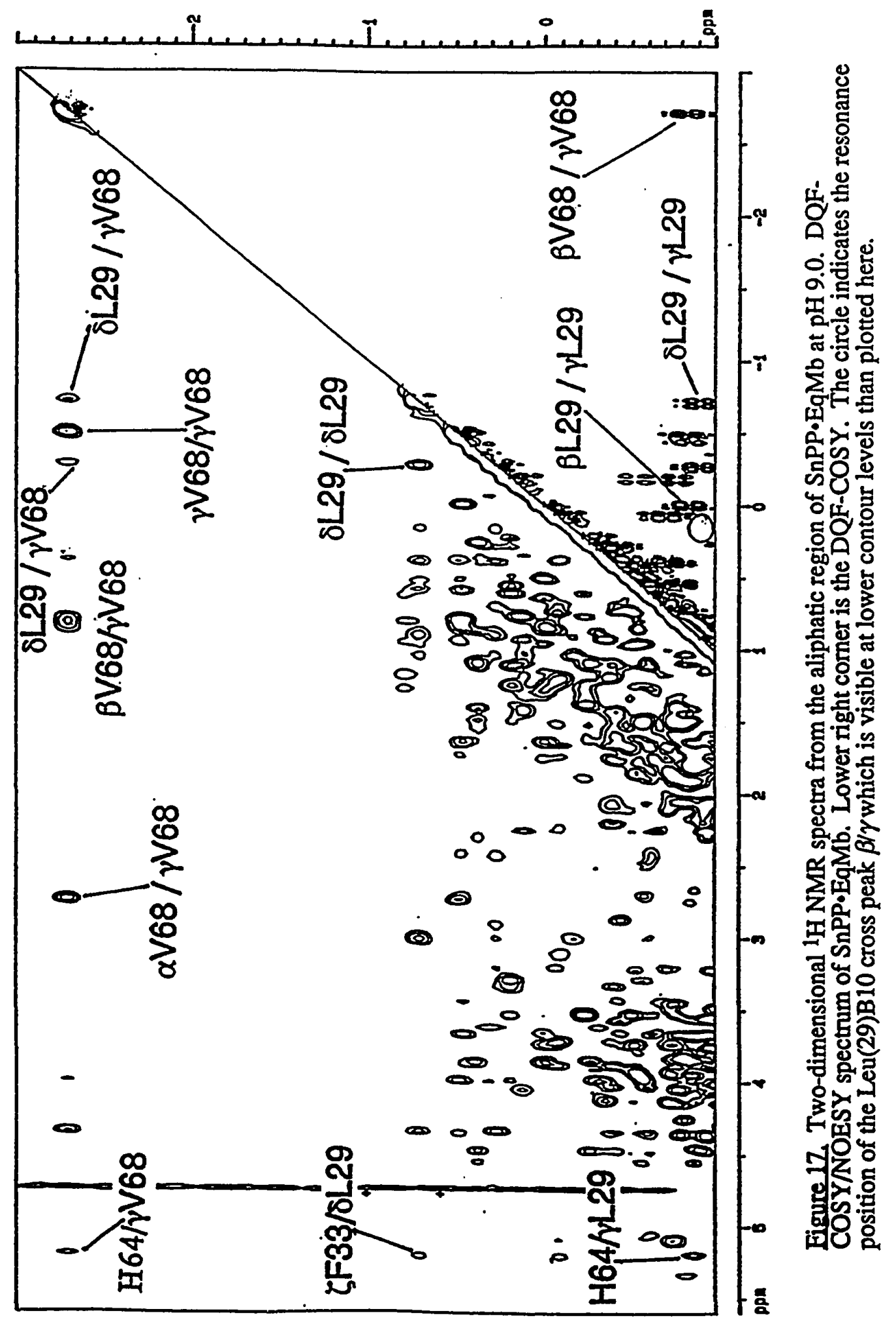




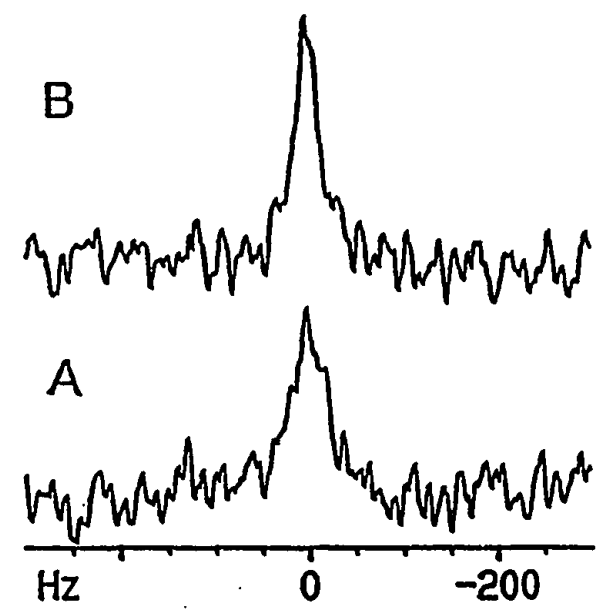

Figure 18. ${ }^{119} \mathrm{Sn}$ spectroscopy of SnPP.EqMb at pH 9.0. (A) Acquired without

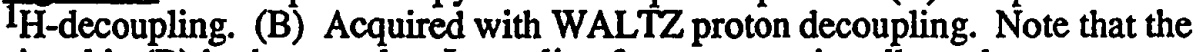
signal in (B) is sharpened as J-coupling from protons is collapsed. 


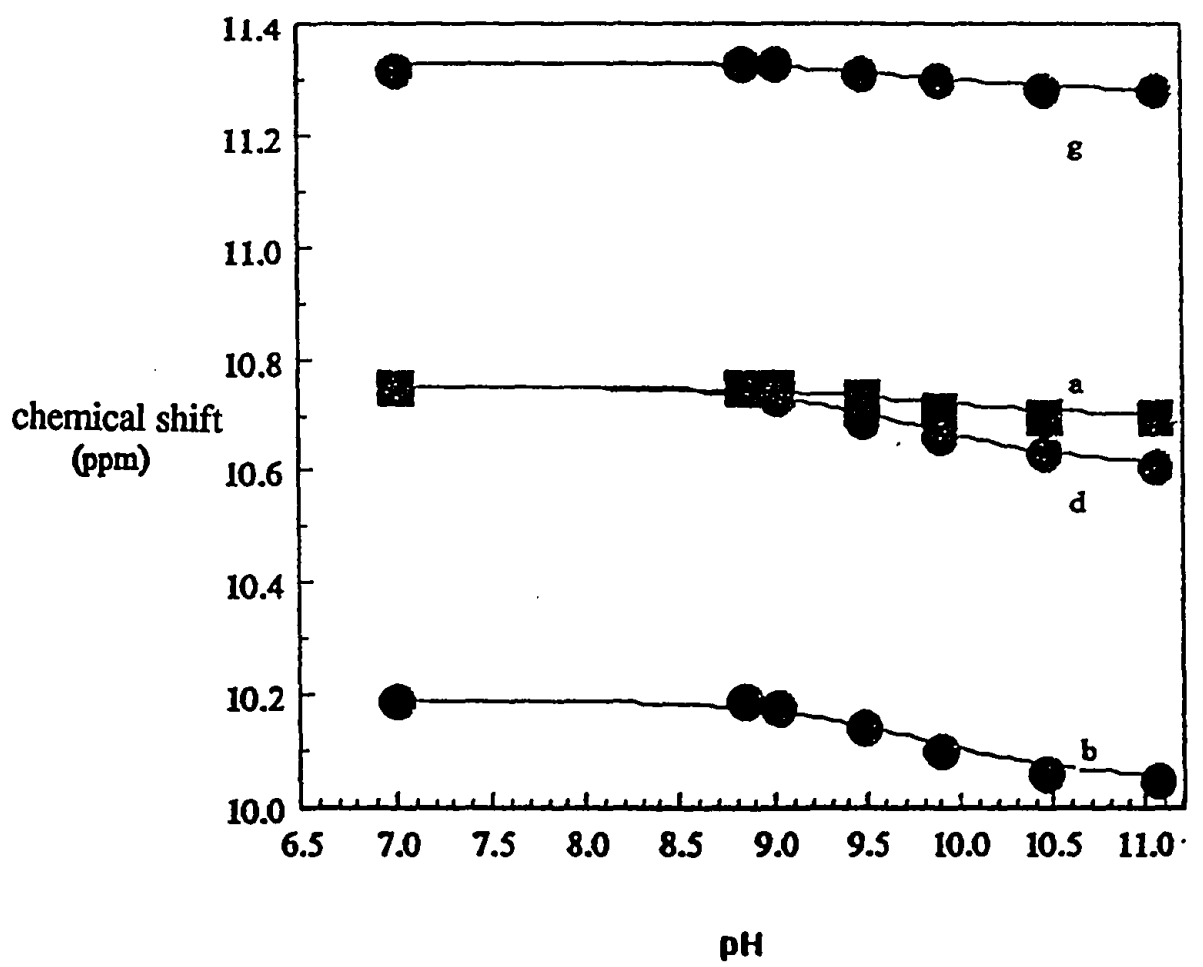

Eigure 19. meso $\mathrm{pH}$ titration of SnPP.EqMb from $\mathrm{pH} 7$ to 10. All four meso protons show strongly perturbed chemical shifts. The $\mathrm{pK}_{\mathrm{a}}$ affecting each meso proton was determined to be 9.8 . 


\section{CHAPTER VI}

\section{PROTON NMR STUDY OF THE INTERACTION OF TIN(IV) PROTOPORPHYRIN IX MONOMERS AND DIMERS WITH APOMYOGLOBIN}

Contributions of the heme prosthetic group to protein structure and stability are of continuing interest (Schweitzer-Stenner et al., 1992; Guiles et al., 1992). The effects of various modifications of parts of the heme have been studied to gain insight into the contribution of the vinyls and the propionates to the equilibrium ratio and reorientation kinetics of the heme-insertion isomers depicted in Figure $12 \mathrm{~A}$ and B in Chapter V (La Mar et al., 1989, 1991; Hauksson et al., 1990), but there has been relatively little done to illuminate the role of the porphyrin metal ion. Efforts to observe the alternative isomer failed in freshly-reconstituted PPIX $\cdot \mathrm{Mb}$, presumably because the complex rapidly attains the equilibrium state, for which there is only one observable porphyrin-insertion isomer (La Mar et al., 1989; Lecomte and Cocco, 1990). This chapter therefore deals with events during the reconstitution of apoMb with SnPP to form the holoprotein, with the goal of finding intermediates sufficiently long-lived to allow direct detection by ${ }^{1} \mathrm{H}$ NMR. Thus interactions between SnPP and apoEqMb, and between dimeric (SnPP) $)_{2}$ and apoEqMb were observed by ${ }^{1} \mathrm{H}$ NMR and optical absorbance spectroscopy. The equilibrium structure for the complex SnPP.EqMb was shown in Chapter V to be analogous to metEqMbH${ }_{2} \mathrm{O}$ (Deeb and Peyton, 1991). Here, a relatively long-lived intermediate within the reconstitution process: $\mathrm{SnPP}+\mathrm{apoEqMb} \rightarrow \mathrm{SnPP} \cdot \mathrm{EqMb}$ is demonstrated . Further, the well-characterized monomer-dimer equilibrium of SnPP (Breslow et al., 1986) discussed in Chapter IV, permits the direct observation of dimeric $(\mathrm{SnPP})_{2}$ interacting with apoMb, but not with the reconstituted protein $\mathrm{SnPP} \cdot \mathrm{Mb}$; this 
interaction was first suggested by Breslow et al. (1986) from kinetics arguments alone. The material presented in this chapter was included in the publication: Deeb, R.S., and Peyton, D.H. (1992) "Proton NMR Study of the Interaction of Tin(IV)Protoporphyrin IX Monomers and Dimers with Apomyoglobin" Biochemistry. 31, 468-474.

\section{MATERIALS AND METHODS}

Preparation of materials was explained in Chapter III. (SnPP) 2 solutions were prepared by allowing the monomeric solution to incubate in the dark at $\mathrm{pH} 7.4$, and the conversion was verified by ${ }^{1} \mathrm{H}$ NMR. Several of the studies here involve non-equilibrium mixtures. Data acquisitions were kept as short as possible in both optical and NMR measurements so as to minimize effects due to averaging. This problem is especially severe for the fast reactions such as the incorporation of $(\mathrm{SnPP})_{2}$ into apoEqMb. In this case, each NMR spectrum was unavoidably averaged over a time period of at least $\sim 0.8$ min; the optical spectra required 3 minutes for each acquisition, but the time between each wavelength is simply the difference between the start of any two acquisitions.

\section{RESULTS}

\section{$1 \mathrm{H} N M R$ Observation of an Intermediate in the Process: SnPP + apoEqMb $\rightarrow$ $\underline{\mathrm{SnPP} \cdot E q M b}$}

The downfield region at $25^{\circ} \mathrm{C}$ and $\mathrm{pH} 10.2$ is shown in Figure $20 \mathrm{~A}$ and $\mathrm{D}$ for apoEqMb, after $\sim 5$ and $\sim 400$ min following mixing with an excess of SnPP. SnPP•EqMb (Chapter V) is the final product, shown in Figure $20 \mathrm{D}$. At early times after mixing (Figure $20 \mathrm{~A}$ ), an intermediate (hereafter called SnPP•EqMb*) is evident from the mesoproton shifts, in that they are different in appearance and chemical shifts from those of 
SnPP.EqMb (Figure 20 D); a resonance from His FG3 is also shifted in SnPP•EqMb*. Saturation transfer via chemical exchange between meso-protons from SnPP and SnPP.EqMb* is shown in Figures $20 \mathrm{~B}$ and $\mathrm{C}$ (note that excess SnPP provides the opportunity to observe chemical exchange between SnPP.EqMb* meso-H's and the free $\mathrm{SnPP}$, and hence assignments can be made for SnPP•EqMb* meso-H's). Analogous saturation transfer by chemical exchange between SnPP and the final species SnPP.EqMb is not observed (Figures $20 \mathrm{E}$ and $\mathrm{F}$ ). The assignment of meso $\mathrm{H}_{y}$ in $\mathrm{SnPP} \cdot \mathrm{EqMb} *$ was straightforwardly related to the SnPP meso $\mathrm{H}_{\gamma}$ by saturation transfer (Figure $20 \mathrm{C}$ ), but overlap between $\mathrm{SnPP}$ meso $\mathrm{H}_{\beta}$ and meso $\mathrm{H}_{\delta}$ resonances introduced an ambiguity into the assignment of meso $\mathrm{H}_{\beta}$ in SnPP.EqMb*. This was however, resolved by the NOE in Figure $20 \mathrm{~B}$ to a porphyrin 4vinyl $\mathrm{H}_{\alpha}$, which dictated the irradiated resonances as arising from the $\mathrm{SnPP} \cdot \mathrm{EqMb} *$ meso- $\mathrm{H}_{\beta}$. meso- $\mathrm{H}_{\delta}$ will show no NOEs to vinyl protons. Figure 21 shows results of a reconstitution of SnPP into apoEqMb at 10 and $25^{\circ} \mathrm{C}$. At the lower temperature, chemical exchange would be attenuated, while any NOEs would become more negative owing to the increased correlation time (slower tumbling) of the protein. In fact, the magnetization transfer from the meso $\mathrm{H}_{\beta}$ proton in SnPP.EqMb* is diminished at lower temperature (compare Figure 21 panel $\mathrm{B}$ at $10^{\circ} \mathrm{C}$ to panel $\mathrm{C}$ at $15^{\circ} \mathrm{C}$ ). The assignments for SnPP in solution (Chapter IV) combined with the saturation-transfer by chemical exchange in Figures 20 and 21 made it possible to assign the meso-proton resonances for SnPP.EqMb* as indicated in Table IV.

$\mathrm{SnPP} \cdot \mathrm{EqMb} *$ has its upfield resonance from $\mathrm{Val}(68) \mathrm{E} 11 \gamma \mathrm{CH}_{3}$ at almost the same chemical shift as does SnPP.EqMb; Figure $22 \mathrm{~A}$ is a reference spectrum recorded at intermediate time to show both species (SnPP•EqMb* and SnPP•EqMb). Figure 22 B shows the NOE difference spectrum resulting from irradiating at the position of the $\mathrm{Val}(68) \mathrm{E} 11 \mathrm{CH}_{3}$ resonance from SnPP-EqMb*. Figure $22 \mathrm{C}$ shows the NOE difference spectrum resulting from irradiating at the position of the $\mathrm{Val}(68) \mathrm{E} 11 \mathrm{\gamma CH}_{3}$ resonance of 
the equilibrium species SnPP-EqMb. Both NOE difference spectra show NOEs to the position of the heme meso $\mathrm{H}_{\delta}$ (although in SnPP•EqMb* the meso $\mathrm{H}_{\delta}$ and $\mathrm{H}_{\alpha}$ overlap), as well as to the indicated $\operatorname{Val}(68) \mathrm{E} 11$ resonances; the position of the $\operatorname{Val}(68) \mathrm{E} 11 \alpha \mathrm{H}$ in Figure $22 \mathrm{~B}$ is somewhat downfield of its equilibrium location at $2.74 \mathrm{ppm}$ (Figure $22 \mathrm{C}$ ). There are also NOEs observed to $\operatorname{Leu}(29) B 10$ analogous to the equilibrium species. It is concluded that SnPP•EqMb* has the distal side of the porphyrin-binding pocket very similar to that of SnPP•EqMb, including predominantly a single porphyrin-insertion isomer.

TABLE IV

\begin{tabular}{lll}
\multicolumn{2}{c}{ NMR CHEMICAL SHIFTS FOR SnPP·EqMb* AND SnPP•EqMb AT $25^{\circ} \mathrm{C}^{\mathrm{a}}$} \\
Assignment & SnPP•EqMb* $(\mathrm{ppm})$ & SnPP•EqMb $(\mathrm{ppm})$ \\
$\alpha$-meso & 10.72 & 10.65 \\
$\beta$-meso & 10.14 & 9.82 \\
$\gamma$-meso & 11.31 & 11.14 \\
$\delta$-meso & 10.70 & 10.65 \\
$4 \mathrm{H}_{\alpha}$ & 8.79 & 8.61
\end{tabular}

aSnPP•EqMb at pH 9.0; SnPP•EqMb* at pH 10.2. bTaken from Chapter V; Deeb and Peyton, 1991

Optical Spectroscopic Observation of an Intermediate in the Process: SnPP + apoEqMb $\rightarrow$ SnPP.EqMb

The spectra in Figure 23 A demonstrate the time dependence for the Soret region from the reconstitution of SnPP into apoEqMb; Figure $23 \mathrm{~B}$ shows the difference spectra for this reaction. This result is reminiscent of the optical spectra obtained for the reconstitution of heme into apoSwMb (Gebe et al., 1989). In that case, however, the initially observed species was a 1:1 mixture of heme-insertion isomers (Jue et al., 1983), 
while here the initially observed species is not a mixture, at least as detected by ${ }^{1} \mathrm{H}$ NMR. The isosbestic point in the optical spectra reflects a conversion of one species to another species, explicitly, SnPP.EqMb* to SnPP•EqMb. The shift in $\lambda_{\max }$ is from $\sim 416 \mathrm{~nm}$ (SnPP•EqMb*, right spectrum in Figure $23 \mathrm{~A}$ ) to $\sim 417 \mathrm{~nm}$ (SnPP•EqMb, left spectrum in Figure $23 \mathrm{~A}$ ); for the free porphyrin, $\lambda_{\max }$ is $\sim 407 \mathrm{~nm}$ (SnPP, pH 10.2) and the band shape is similar to those shown in Figure 23 A (Breslow et al., 1986).

$1_{H}$ NMR Observation of an Intermediate in the Process: $(\mathrm{SnPP})_{2}+2 \mathrm{apoEqMb} \rightarrow$ 2SnPP.EqMb

Figure 24 presents the meso-proton region for the reaction between $(\mathrm{SnPP})_{2}$ and apoEqMb at $\mathrm{pH}$ 7.5. Figure 24 A shows the meso region of a ${ }^{1} \mathrm{H}$ NMR spectrum of $(\mathrm{SnPP})_{2}$. Figure $24 \mathrm{~B}$ was acquired 2-2.8 min after combining $(\mathrm{SnPP})_{2}$ with apoMb. The meso-proton peaks from (SnPP), now marked by asterisks $\left(^{*}\right)$, are poorly resolved and generally shifted upfield relative to $(\mathrm{SnPP})_{2}$ in Figure $24 \mathrm{~A}$. Clearly, the porphyrin is interacting with apoEqMb but still is a dimer, as indicated by the meso-proton chemical shifts which reflect the ring-current shift from the alternate porphyrin ring. Further evidence, from visible spectroscopy, that $\mathrm{SnPP}$ remains a dimer in $(\mathrm{SnPP})_{2} \cdot \mathrm{EqMb}$ is presented below. Figure $24 \mathrm{C}$ was acquired $10-13 \mathrm{~min}$ after combining $(\mathrm{SnPP})_{2}$ with apoEqMb. A significant amount of SnPP•EqMb is now observed; the remaining $(\mathrm{SnPP}){ }_{2} \cdot \mathrm{EqMb}$ signals have a different overall appearance in Figure $24 \mathrm{C}$ than in Figure $24 \mathrm{~B}$. (SnPP) $2^{\bullet E q M b}$ is therefore heterogeneous. Figure $24 \mathrm{D}$, acquired $118-121 \mathrm{~min}$ after combining (SnPP) 2 with apoMb, showed only the equilibrium species SnPP.EqMb. A second aliquot of (SnPP) 2 added to the product of Figure $24 \mathrm{D}$ produced the spectrum of Figure $24 \mathrm{E}$, which can be described as an approximate linear combination of Figure 24 panels $A$ and D: (SnPP)2 appeared not to interact with SnPP-Mb. 
Another species in Figure $24 \mathrm{C}$ is indicated with arrows; this new species is designated as SnPP•EqMb'. SnPP•EqMb' cannot be SnPP•EqMb* because its characteristic meso $\mathrm{H}_{\gamma}$ resonance is missing from $\sim 11.1 \mathrm{ppm}$. A pH-jump experiment from a high $\mathrm{pH} \sim 9$ to neutral $\mathrm{pH}$ demonstrated that $\mathrm{SnPP} \cdot \mathrm{EqMb} *$ is converted to SnPP•EqMb within $<1 \mathrm{~min}$ with no detectable SnPP•EqMb` formed, therefore, $\mathrm{SnPP} \cdot E q M b^{\prime}$ cannot arise from a $\mathrm{pH}$ effect. Furthermore, SnPP•EqMb' cannot originate from $(\mathrm{SnPP})_{2}$ or $(\mathrm{SnPP})_{2} \cdot \mathrm{EqMb}$ because reconstitution of excess apoEqMb at neutral $\mathrm{pH}$ with a small aliquot of SnPP produced SnPP.EqMb' without detectable formation of $(\mathrm{SnPP})_{2}$ or $(\mathrm{SnPP})_{2} \cdot \mathrm{EqMb}$. Therefore, SnPP•EqMb' and SnPP•EqMb* are different species. SnPP•EqMb' unfortunately was never present in sufficient quantity to allow NOEs to be observed from its meso $\mathrm{H}$ resonances. The identity of $\mathrm{SnPP} \cdot \mathrm{EqMb}^{\circ}$ is discussed below.

Optical Spectroscopic Observation of the Kinetics of the Process: $\left(\mathrm{SnPP}_{2}+2 \mathrm{apoEqMb}\right.$ $\rightarrow 2$ SnPP.EqMb

The spectra in Figure 25 A demonstrate the time course for the Soret region from the reconstitution of $(\mathrm{SnPP})_{2}$ into apoEqMb; Figure $25 \mathrm{~B}$ shows difference spectra for this reaction. ApoMb was in excess, to insure that the initial species formed is $(\mathrm{SnPP})_{2} \cdot \mathrm{EqMb}$, as seen in the NMR spectrum in Figure $24 \mathrm{~B}$; the dimer band is broadened, lowered in intensity, and at shorter wavelength, $387 \mathrm{~nm}$ than SnPP or SnPP.EqMb. These are characteristic of the porphyrin being a dimer $\left[\lambda_{\max } \sim 386 \mathrm{~nm}\right.$ $\left.(\mathrm{SnPP})_{2} ; \lambda_{\max } \sim 407 \mathrm{~nm}(\mathrm{SnPP})\right]$. The final species in Figure $25 \mathrm{~A}$ is characteristic of SnPP•EqMb $\left(\lambda_{\max } \sim 416 \mathrm{~nm}, \mathrm{pH} 7.1\right)$. Although the NMR spectra shown in Figure $24 \mathrm{~B}$ and $\mathrm{C}$ dictate that $(\mathrm{SnPP})_{2} . \mathrm{EqMb}$ must be heterogeneous and its components change in ratio with time, the optical spectra are quite insensitive to this. Conversion to $\mathrm{SnPP} \cdot \mathrm{EqMb}$ must occur with loss of dimer, and the rate of dimer dissociation is enhanced 
in the presence of apoEqMb (by a factor of $\sim 2$; Figure 26). It is possible that upon the interaction of $(\mathrm{SnPP})_{2}$ with the proximal side of the porphyrin pocket, the formation of HisF8-Sn bond speeds up the dissociation of the dimer. This mechanism is shown in Figure 27.

\section{DISCUSSION}

Structural information can be inferred from the following observations about $\mathrm{SnPP} \cdot \mathrm{EqMb}$. First, the chemical shifts and NOEs from upfield $\mathrm{Val}(68) \mathrm{E} 11 \mathrm{CH}_{3}$ are virtually identical from SnPP.EqMb and SnPP·EqMb* (Figure 22) indicating that the distal side of the porphyrin-binding pocket is largely intact in the vicinity of this residue. Second, the off-rate for SnPP from SnPP.EqMb* is estimated (from the magnitude of saturation transfer and $\mathrm{T}_{1}$ sel of free $\mathrm{SnPP}$ meso-protons, $\sim 0.5 \mathrm{~s}$ ) to be $\sim 1 \mathrm{~s}^{-1}$ (Sandstrom, 1982) indicating that the proximal His(64)E7-Sn bond is almost certainly missing. Third, SnPP.EqMb* does not have His(97)FG3 $\gamma \mathrm{H}$ at its usual chemical shift (compare Figure 20 panels A and D), therefore, the salt bridge between His(97)FG3 and the heme 7propionate may not be formed or may be in a perturbed conformation. The resulting picture of SnPP.EqMb* has SnPP inserted into the globin in approximately the same way as in SnPP•EqMb or the heme in Mb, but with the proximal His(93)F8 not coordinated to $\mathrm{Sn}$, and the 7-propionate to His(97)FG3 H-bond either missing or different from its native structure. Figure 28 provides a general comparison of the $6^{\text {th }}$ ligands on the porphyrin between SnPP·EqMb* and SnPP·EqMb. Chapter V (Figure 18) provided evidence that in $\mathrm{SnPP} \cdot \mathrm{EqMb}$, the Sn-His(93)F8 bond is indeed present, as is the 7-propionate-His(97)FG3 salt bridge as suggested by the chemical shift of the His(97)FG3 $\gamma \mathrm{H}$ resonance. The distal position appears to be occupied by water, as revealed by meso-proton $\mathrm{pH}$-titration behavior discussed in Chapter V. 
La Mar et al. (1989), and Lecomte and Cocco (1990) tried to observe the formation of porphyrin-insertion isomers in freshly prepared PPIX·SwMb and PPIX EqMb, respectively. The lack of the proximal His(93)F8-metal bond was invoked to explain rapid approach to an equilibrium distribution of heme-insertion isomers, so that only a single porphyrin-insertion isomer could be observed in the time required to obtain an NMR spectrum. The observations in this chapter support this idea of rapidly exchanging porphyrin-insertion isomers if the His(93)F8-metal bond is missing. In fact, the estimated rate of SnPP dissociation from SnPP•EqMb*, $\mathrm{K}_{\mathrm{off}} \sim 1 \mathrm{~s}^{-1}$, is a lower bound for porphyrin-insertion isomer redistribution for SnPP.EqMb* because porphyrin reorientation is not supposed to require complete dissociation from the globin (La Mar et al., 1984). The notion that metal-His(93)F8 bond might be relatively slow to form in some cases is supported by optical studies of the formation of metEqMbCN from hemin dicyanide and apoEqMb (Yee and Peyton, 1991; Kawamura-Konishi et al., 1988), for which the step assigned as formation of the Fe-His(93)F8 bond was rate limiting.

La Mar et al. (1989) found that the position occupied by the 6-propionate in native $\mathrm{Mb}$ forms the initial H-bond when reconstituting SwMb with either 6-methyl-6-despropionate hemin (hemin without the propionate on the $6^{\text {th }}$ carbon) or 7-methyl-7-despropionate hemin. The residue involved in this salt bridge is $\operatorname{Arg}(45) C D 3$ (Lys(45)CD3 in EqMb); this residue's side chain has been found to be disordered in the CO complex of SwMb (Kuriyan et al., 1986), and this disorder may be a part of the mechanism of ligand binding by the globin. The observations from Figure 20 show the $\varepsilon H$ from His(97)FG3, which forms the salt bridge to the 7-propionate, is absent from its usual resonance position in SnPP.EqMb*, this resonance is observed only when the final species, SnPP•EqMb, is formed. His(97)FG3 is separated from His(93)F8 by only 3 residues, and the F-helix is likely not in the native position in SnPP.EqMb*, as may be the case in apoEqMb (Hughson et al., 1990; Cocco and Lecomte, 1990). No assignments 
are found for Lys(45)CD3 in SnPP.EqMb, so there is no direct evidence about the state of the salt bridge to the 6-propionate in SnPP-EqMb*. However, the adjacent residue, $\mathrm{His}(64) \mathrm{E} 7$ is in about the same position as in SnPP.EqMb (the peak from the $\varepsilon H s$ are at approximately the same position throughout as seen in Figure 22), strongly ring-current-shifted by the porphyrin, so it is reasonable that $\operatorname{Lys}(45) C D 3$ is also approximately in its native spatial position, with the salt bridge to the 6-propionate formed.

The insertion of the ( $\mathrm{SnPP})_{2}$ into the apoMb is perhaps the most surprising part of this work because the X-ray crystal structures for Mb have the native porphyrin (heme) tightly enclosed within the protein as seen in Figure 29, so there seems to be no room for species as wide as a porphyrin dimer (Evans and Brayer, 1988). Both the meso ${ }^{1} \mathrm{H}$ NMR chemical shifts of (SnPP) ${ }_{2}$ and $\mathrm{X}$-ray structures of $\mu$-oxo metalloporphyrin oligomers (Hoffman et al., 1972) suggest that the width of the dimer is in the $\sim 6 \AA$ (Sn-to-Sn centers separated by $\sim 4 \AA$ ). However, apoMb has a reduced helical content relative to the holoprotein (Breslow et al., 1965; Harrison and Blout, 1965), and it may be that the F-helix is partly or largely unfolded [Hughson et al. (1990) and above]. Such a structural perturbation could provide sufficient room and/or flexibility at the nascent heme pocket in apoMb for ( $\mathrm{SnPP})_{2}$ to interact. An analogous structure involving heme may be a detectable intermediate by the methods analogous to those of Kawamura-Konishi et al. (1988) if a significant amount of heme were to be dimer, rather than higher aggregates. For the present case, it is fortunate that the monomer-dimer equilibrium of SnPP is slow enough to allow the monitoring of such changes directly by NMR methods.

The species SnPP.EqMb' seen in Figure $24 \mathrm{C}$ and marked by arrows, is not detected when reconstitutions are carried out at high $\mathrm{pH}$. The $10.17 \mathrm{ppm}$ resonance may be from the meso $\mathrm{H}_{\delta}$ of the porphyrin-insertion isomer shown in Figure $12 \mathrm{~B}$ in Chapter V; the chemical shift interchange of meso-protons under an $\alpha, \gamma$-meso axis formal rotation in 
diamagnetic hemoglobins has been reported (Cooke and Wright, 1987; La Mar et al., 1989). Also as in those reports, there is evidence of the two porphyrin-insertion isomers as an additional resonance from upfield $\mathrm{Val}(68) \mathrm{E} 11 \mathrm{\gamma CH}_{3}$ (Jue et al., 1983). The identified SnPP.EqMb' peaks correspond well to the pattern of the minor heme-insertion isomer peaks of SwMbCO (La Mar et al., 1989; 1983): minor peak Val(68)E11 $\mathrm{\gamma CH}_{3}$ downfield of the major peak, minor peak meso $\mathrm{H}_{\gamma}$ just upfield of the major meso $\mathrm{H}_{\beta}$ peak, and minor meso $\mathrm{H}_{\beta}$ peak apparently overlapping major meso $\mathrm{H}_{\gamma}$ peak. As noted above, it is unfortunate that $\mathrm{SnPP} \cdot \mathrm{EqMb} \mathrm{b}^{\prime}$ is present in too low an amount at equilibrium and disappears too rapidly after reconstitution to permit NOE studies. Nevertheless, the observation of SnPP.EqMb' only at neutral $\mathrm{pH}$ is reasonable for the alternate porphyrin-insertion isomer because the ligand water, as opposed to hydroxy, could be replaced by His(93)F8 (Figure 28) quickly enough to trap both porphyrin-insertion isomers depicted in Figure $12 \mathrm{~A}$ and $\mathrm{B}$.

Cowan and Gray (1989) suggested that splitting of Q-bands in the 450-650 $\mathrm{nm}$ region of the optical spectrum of metal-substituted porphyrin reconstituted SwMb, including SnPP.SwMb, can be attributed to the presence of the porphyrin-insertion isomers. Although Cowan and Gray used SwMb, the Q-bands for SnPP.EqMb are nearly identical. Previous work discussed in Chapter V showed that there is only one SnPP-insertion isomer in equilibrium SnPP.EqMb, at least as detectable by ${ }^{1} \mathrm{H}$ NMR. In spectra of samples aged at least 12 hours, recorded to very high signal-to-noise ratio, no $\mathrm{SnPP} \cdot \mathrm{EqM} b^{\prime}$ is detected: SnPP•EqMb', the only species observed from above experiments, could be the porphyrin-insertion isomer depicted in Figure $12 \mathrm{~B}$, and is present at most to a few percent at equilibrium. Therefore, the amount of Q-band splitting in SnPP•Mb must be due in major part to factors other than the presence of porphyrin-insertion isomers, although some minor contribution by porphyrin-insertion isomers cannot be excluded. A small change occurs in the 450-650 nm region of the 
optical spectrum when the reconstitution is performed with $\mathrm{SnPP}$ (Figure 30), during the time frame that SnPP.EqMb' disappears from Figure 24. A similar result has been found for FePPIX (Gebe et al., 1989) and attributed formation and subsequent redistribution of hemin-insertion isomers. DiFeo and Addison (1991) reached a similar conclusion for iron porphyrin-globin complexes, that Q-band splitting does not arise from hemeinsertion isomers in that system. They found that the splitting in the $\alpha / \beta$ region of the optical spectrum is different in the presence of insertion isomers.

Structural characterization of a species analogous to $\mathrm{SnPP} \cdot \mathrm{EqMb} *$ in reconstitution reactions involving heme has not been reported. The reason for this is the fast rate for formation of the Fe-His(93)F8 bond (Kawamura-Konishi et al., 1988). Only a very slow to dissociate ligand, such as cyanide, might afford the opportunity to observe such a species by ${ }^{1} \mathrm{H}$ NMR. It has not been possible to detect such a species directly. However, the ratio of heme-insertion isomers from the reaction between apoMb and hemin dicyanide does, in fact reflect a heme-insertion isomer reorientation occurring before Fe-His(93)F8 bond formation (Yee and Peyton, 1991). Therefore, SnPP•EqMb* is an interesting and long-lived model.for a very short-lived species along the heme-insertion reaction pathway for $\mathrm{Mb}$. 

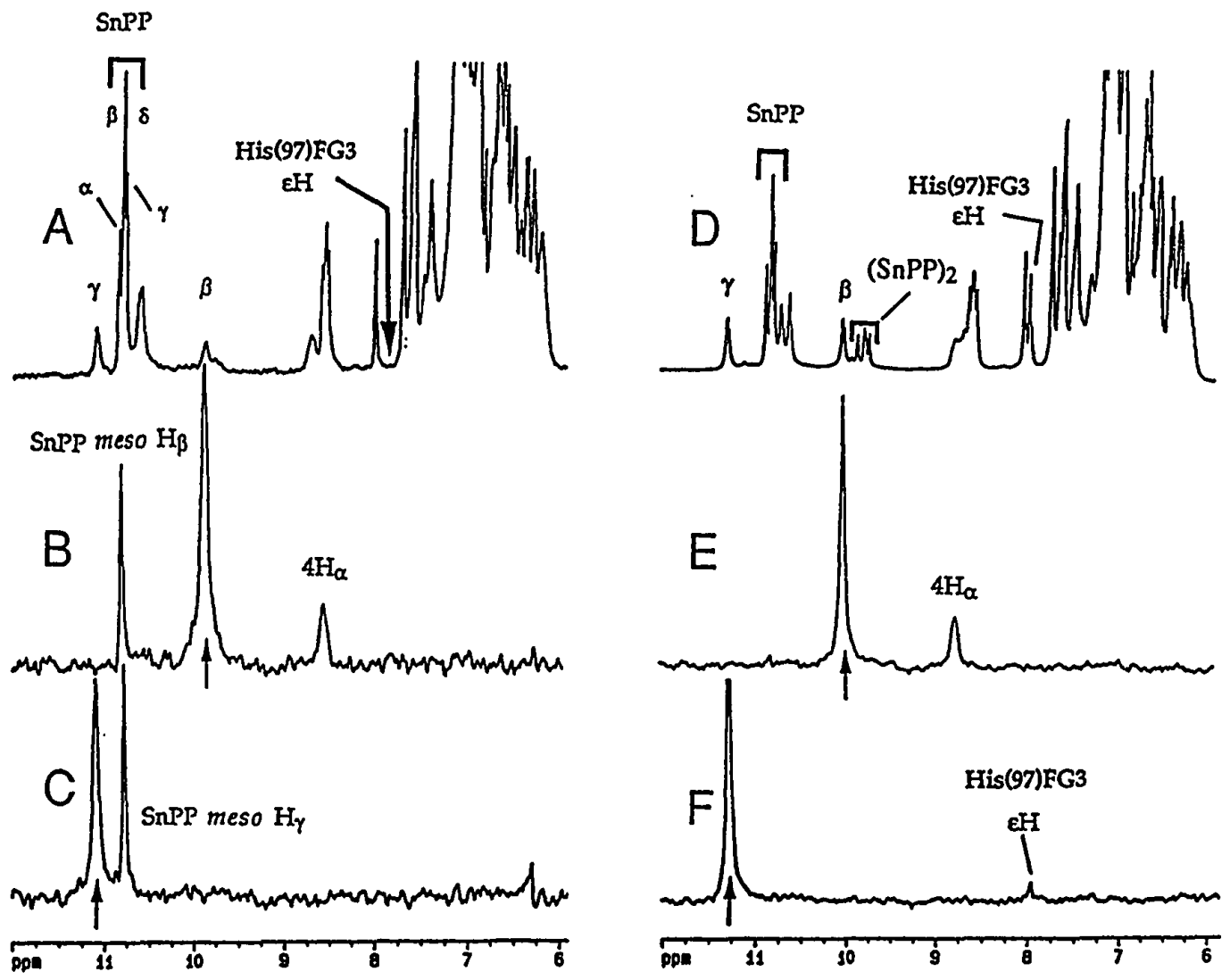

Figure 20. Saturation-transfer for SnPP.EqMb* and SnPP.EqMb in the presence of excess SnPP monomer at $\mathrm{pH} 10.2$, and $25^{\circ} \mathrm{C}$. (A) Reference spectrum recorded immediately after reconstitution showing the missing $\varepsilon \mathrm{H}$ from His(97)FG3. (B) Difference spectrum resulting from irradiating at the position labeled $\beta$ in reference trace (A). Note the NOE to the vinyl $\mathrm{H}_{\alpha}$ and the saturation transfer to the free monomer mesoH $\beta$. (C) Difference spectrum resulting from irradiating at the position labeled $\gamma$ in reference trace (A). Magnetization transfer is observed to the free monomer mesoH $\mathrm{H}_{\gamma}$. (D) Reference spectrum recorded after the sample from trace $(A)$ has attained equilibrium ( $400 \mathrm{~min}$ after reconstitution). $\varepsilon H$ from His(97)FG3 is present. (E) NOE difference spectrum resulting from irradiating the mesoH $\mathrm{H}_{\beta}$ of $\mathrm{SnPP} \cdot \mathrm{EqMb}$. (F) NOE difference spectrum resulting from irradiating the meso $\mathrm{H}_{\gamma}$ of SnPP-EqMb. The expected NOEs to Vinyl $\mathrm{H}_{\alpha}$ from mesoH $\beta$ and to $\mathrm{EH}$ of His(97)FG3 from mesoH $\gamma$ are present, but there is no saturation-transfer to free SnPP meso Hs. 


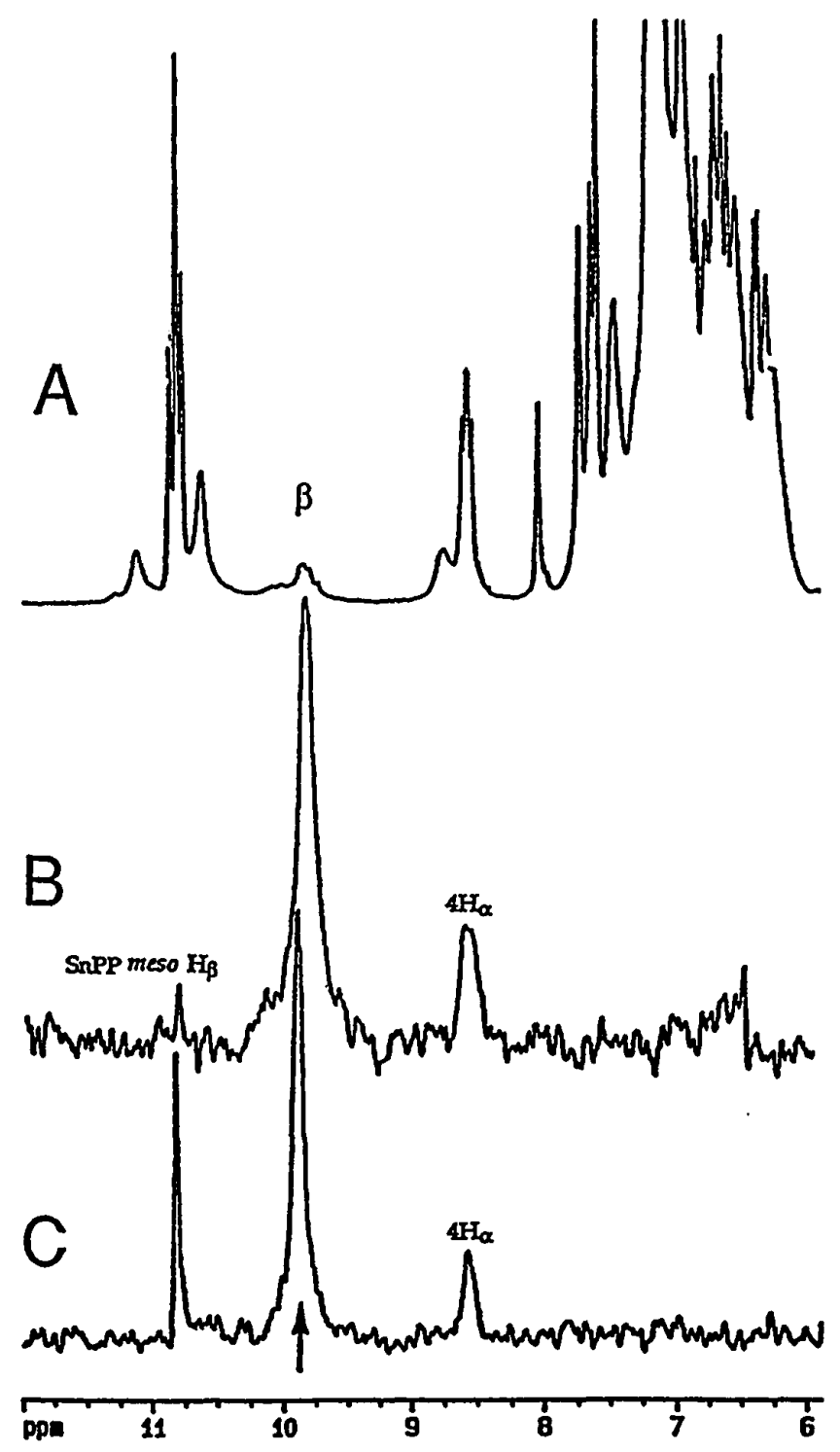

Eigure 21. Saturation-transfer difference spectra for SnPP-EqMb* in the presence of excess SnPP monomer at pH 10.2 , at $10^{\circ}$ and $25^{\circ} \mathrm{C}$. (A) Reference spectrum recorded immediately after reconstitution. (B) Difference spectrum resulting from irradiating at meso $\mathrm{H}_{\beta}$ of $\mathrm{SnPP} \cdot \mathrm{EqMb}^{*}$ at $10^{\circ} \mathrm{C}$. (C) Difference spectrum resulting from irradiating at mesoH $\mathrm{H}_{\beta}$ of $\mathrm{SnPP} \cdot \mathrm{EqMb}^{*}$ at $25^{\circ} \mathrm{C}$. Note the diminished saturation-transfer at the lower temperature (trace B) and the increased NOE to heme vinyl $4 \mathrm{H}_{\alpha}$. 


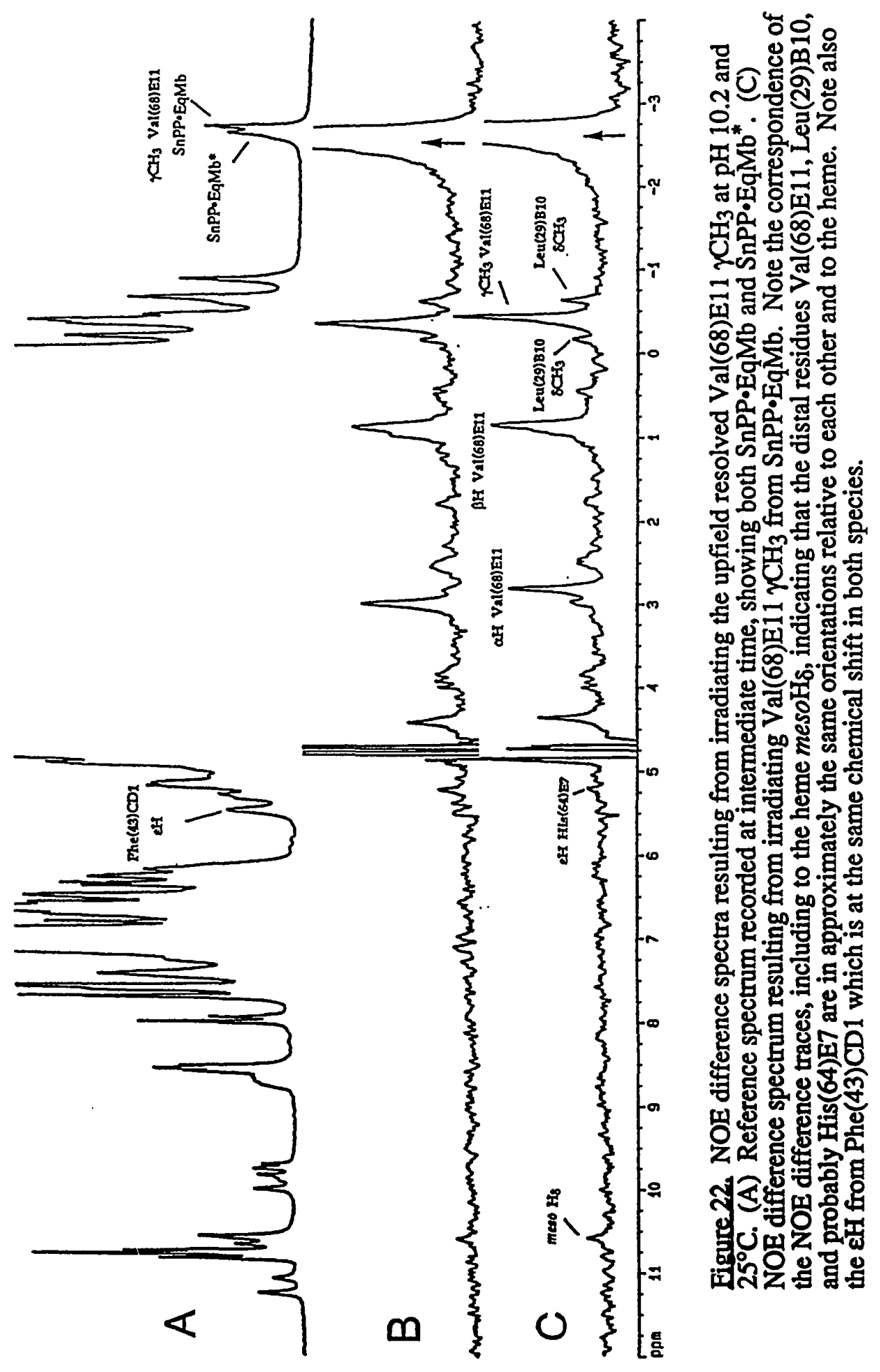




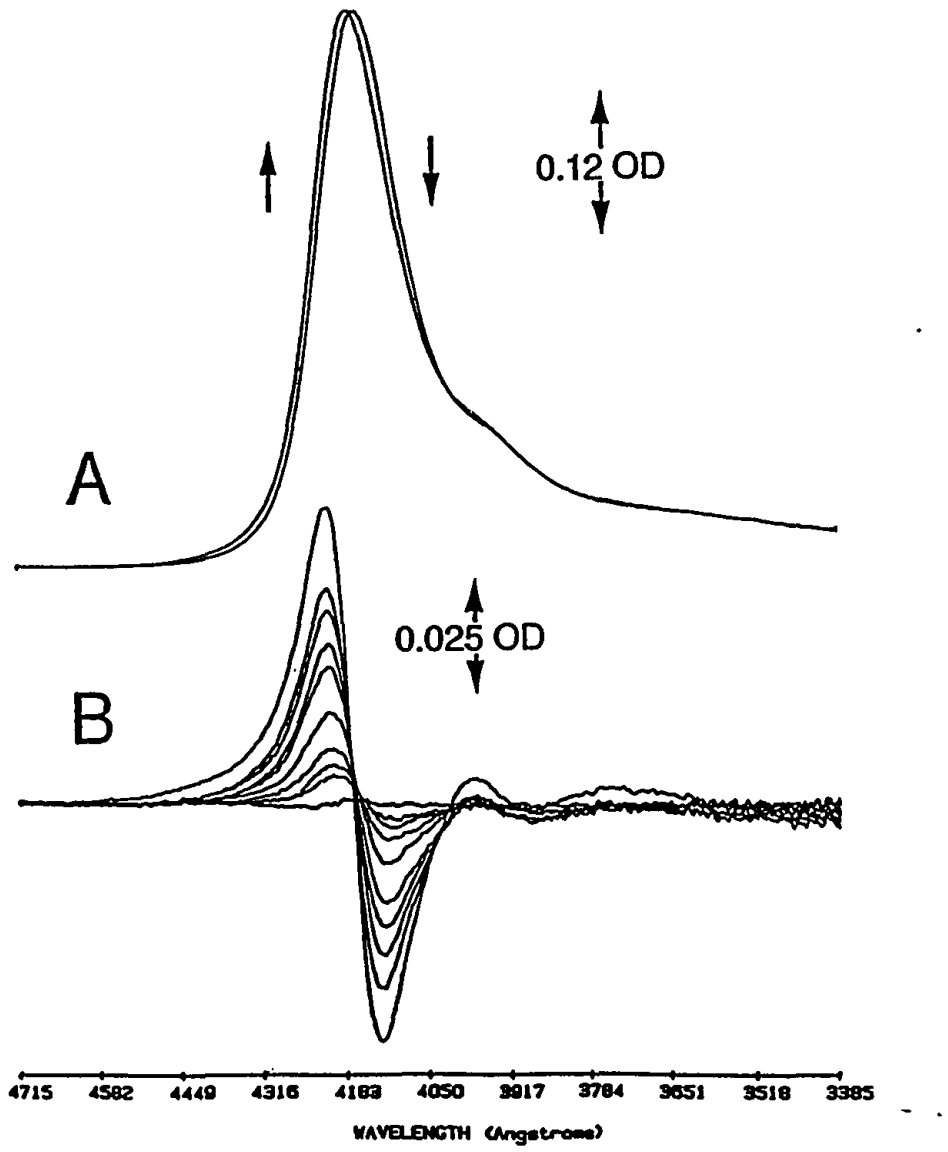

Eigure 23. Optical spectra of the Soret region which reflect the conversion of SnPP.EqMb to SnPP.EqMb at pH 10.0 and $25^{\circ} \mathrm{C}$. (A) Spectra recorded at 1 (right) and 132 (left) min after reconstitution. The right spectrum corresponds to the species reported in Figure 20 (A), while the left spectrum corresponds to SnPP-EqMb, reported in Figure 20 (D). (B) Difference spectra resulting from subtracting spectra recorded at various times from the final spectrum (132 $\mathrm{min}$ ). The rate constant for this process was calculated: $\mathrm{K} \sim 4 \times 10^{-2} \mathrm{~min}^{-1}$. 

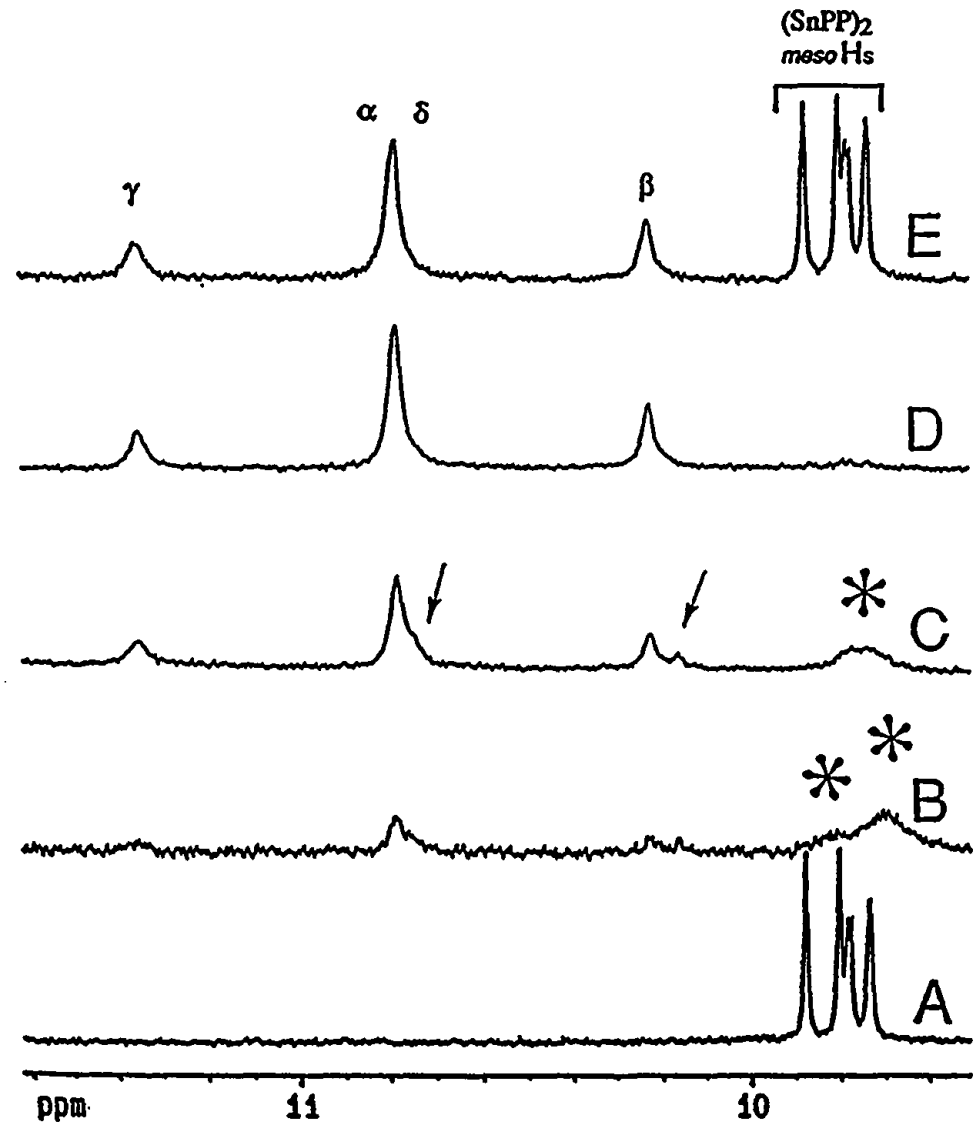

Fiqure 24. ${ }^{1} \mathrm{H}$ NMR observation of the incorporation of (SnPP) ${ }_{2}$ into apoEqMb in the downfield region at $\mathrm{pH} 7.5$ and $25^{\circ} \mathrm{C}$. (A) Spectrum of (SnPP)2. (B-D) Spectra recorded at $2.5,12$, and $120 \mathrm{~min}$ after mixing (SnPP) 2 with apoEqMb. (E) Spectrum recorded after adding a second equivalent of (SnPP) 2 to the sample from (D). Note that (SnPP) 2 appears not to interact with SnPP•EqMb (E), but interacts with apoEqMb (B-D). 


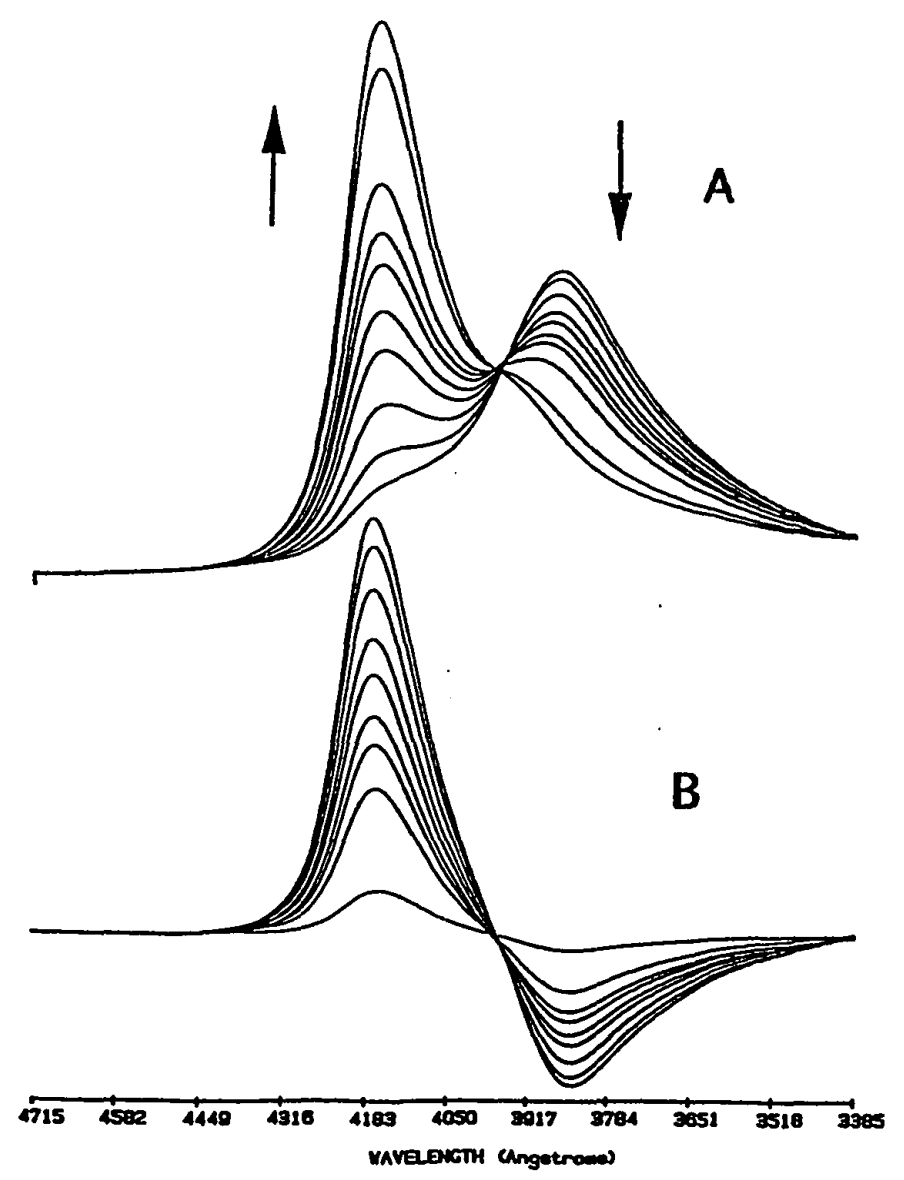

Eigure 25. Optical spectra of the Soret region which reflect the conversion of $(\mathrm{SnPP})_{2} \cdot \mathrm{EqMb}$ to SnPP-EqMb at $\mathrm{pH}$ 7.1. (A) Spectra recorded at various times after reconstitution. Note that the first spectrum corresponds to the species shown in Figure 24 (B), while the last spectrum corresponds to SnPP.EqMb. The species shown in Figure 24 (D). (B) Difference spectra resulting from subtracting spectra recorded at various times from the final spectrum $(258 \mathrm{~min})$. The rate constant for this process was calculated: $\mathrm{K} \sim 2 \times 10^{-2} \mathrm{~min}^{-1}$. 


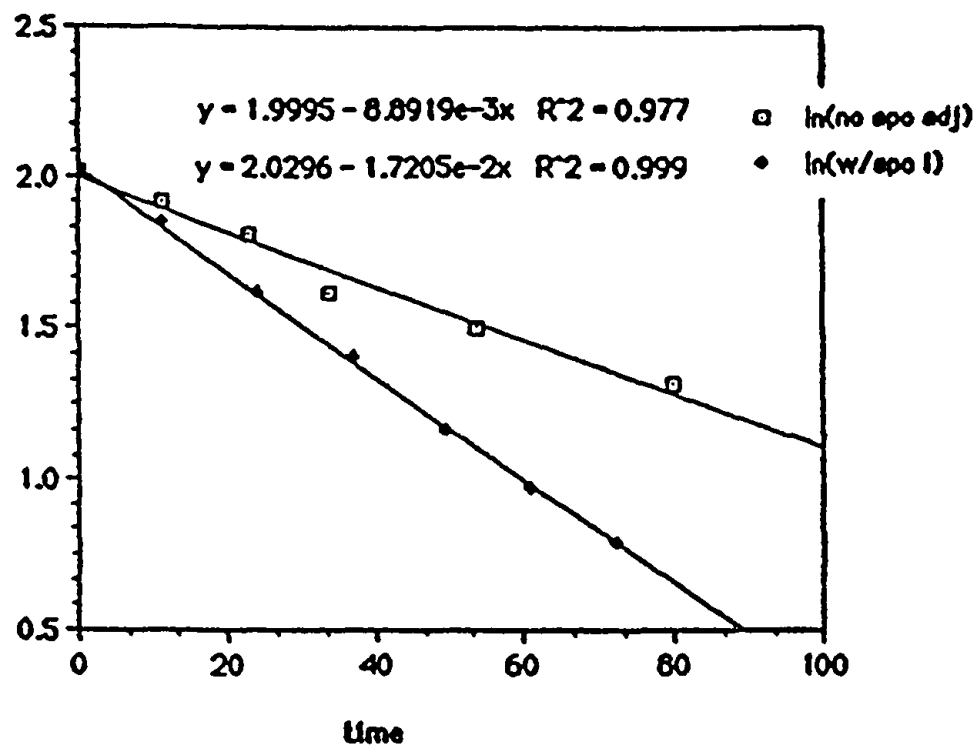

Figure 26. A graph showing the dissociation rate of $(\mathrm{SnPP})_{2}$ in the presence of apoMb $\left(1.7 \times 10^{-2} \mathrm{~min}^{-1}\right)$ and without apoMb $\left(8.8 \times 10^{-3} \mathrm{~min}^{-1}\right)$. The rate of dimer dissociation is enhanced in the presence of the protein. 

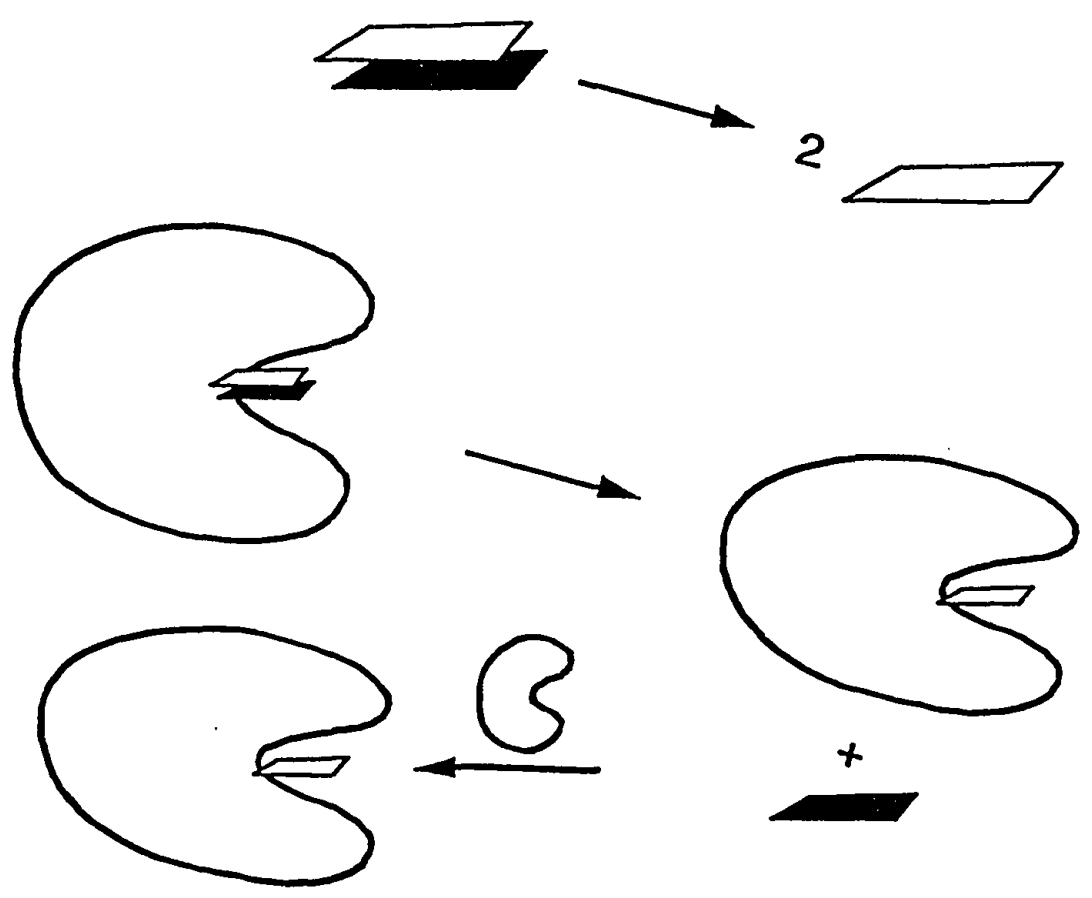

Eigure 27. A schematic diagram illustrating the mechanism of dimer interaction with apoMb. The dimer dissociates following the insertion of the dimer into the apo-pocket. 


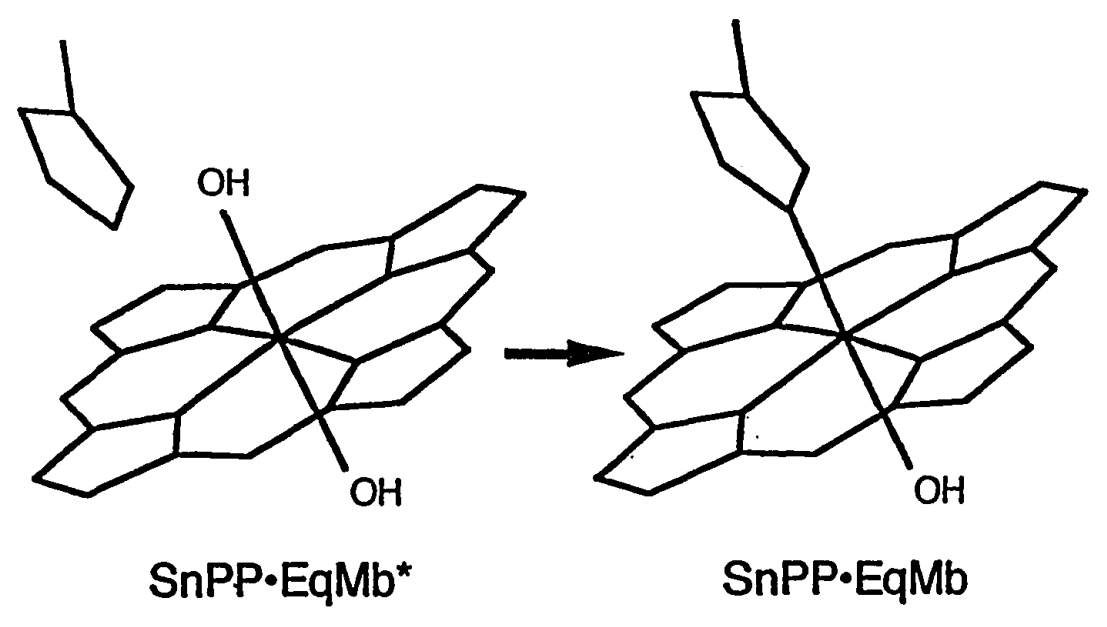

apoMb + SnPP $\longrightarrow$ SnPP-EqMb* $\rightarrow$ SnPP-EqMb

Eigure 28. A schematic diagram illustrating the two species $\mathrm{SnPP} \cdot \mathrm{EqM} \mathrm{b}^{*}$ and $\mathrm{SnPP} \cdot \mathrm{EqM}$. Notice that the $6^{\text {th }}$ ligand on the porphyrin ring of $\mathrm{SnPP} \cdot \mathrm{EqM} \mathrm{B}^{*}$ is an $\mathrm{OH}$ group at high $\mathrm{pH}$ which will be replaced by a histidine ligand at equilibrium (SnPP-EqMb). 


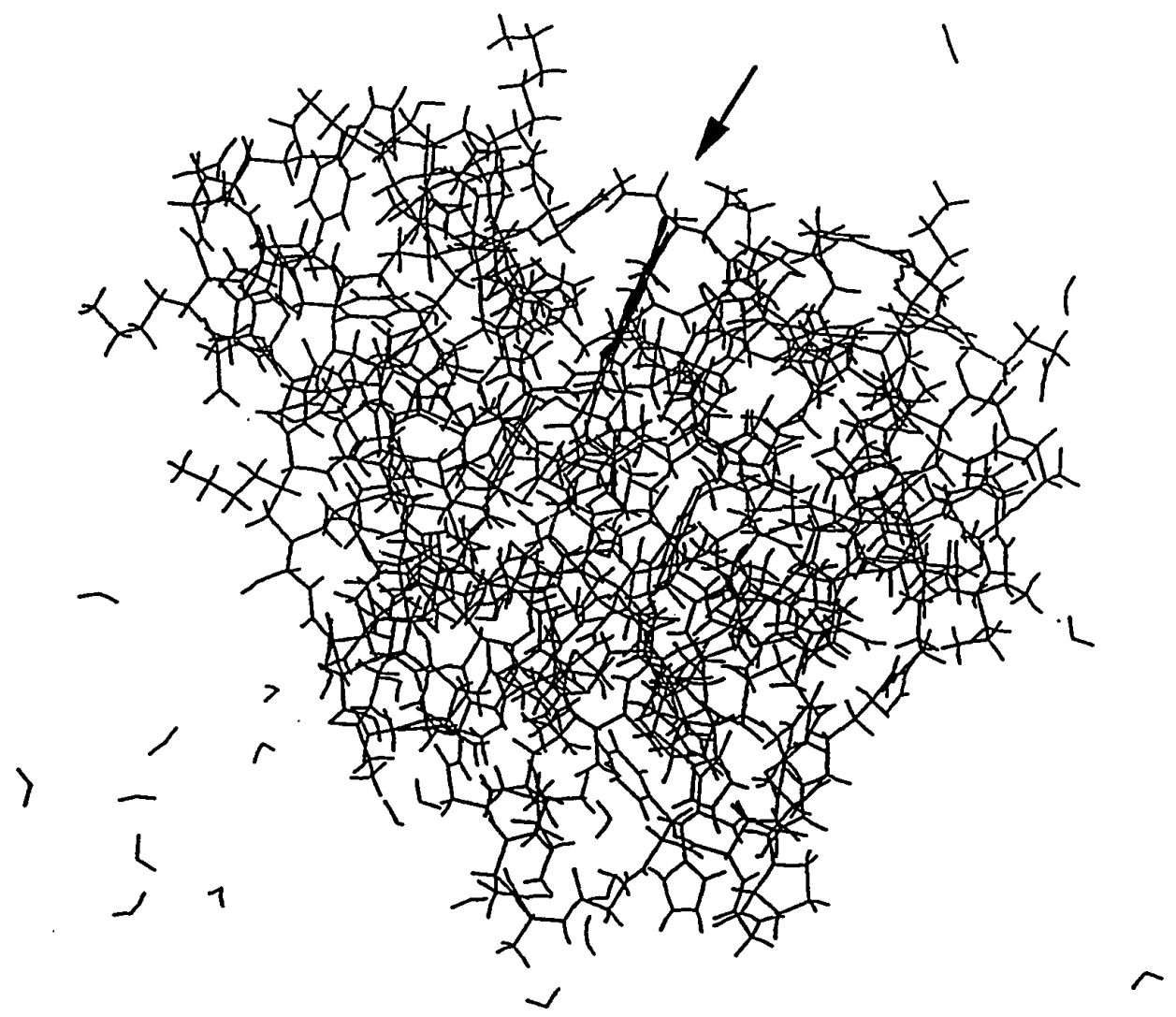

Figure 29. An illustration of the tight fit of the Myoglobin pocket (Evans and Brayer, 1988). An arrow points to the location of the porphyrin ring. This is to emphasize the uniqueness of dimer insertion into the apoMb pocket. 


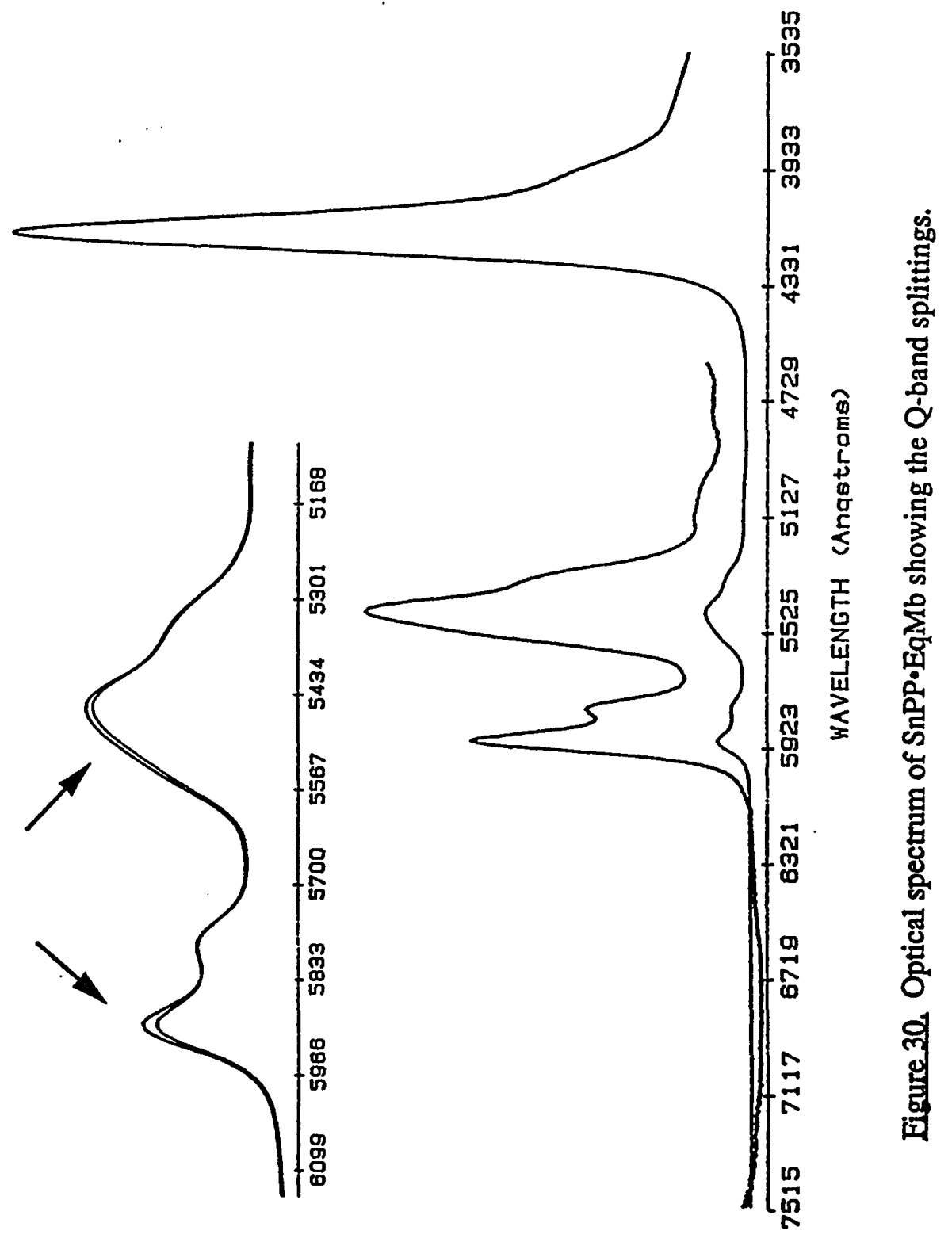




\section{CHAPTER VII}

\section{CHARACTERIZATION OF HEMOPEXIN BY SDS-POLYACRYLAMIDE, GEL ELECTROPHORESIS AND OPTICAL SPECTROSCOPY}

Free-radical reactions have been implicated in cerebral trauma as well injury to lungs, livers and kidneys in humans (Halliwell, 1987). Heme is one example of a biological molecule which is able to participate in oxygen-radical reactions that can lead to the degradation of proteins, carbohydrates and DNA (Tappel, 1955; Aft and Mueller, 1984, 1985). Its toxicity is especially critical in times of excessive hemolysis. A need for means by which this heme activity is removed can therefore be invisioned. Hemopexin $(\mathrm{Hx})$ is a antioxidant that acts by removing heme from circulation. $\mathrm{Hx}$ is a protein that is present at plasma concentration $\sim 0.5 \mathrm{mg} / \mathrm{mL}$ (Muller-Eberhard, 1988) and has two striking properties: $\mathrm{Hx}$ inhibits heme-catalyzed oxidation reactions of at least lipids by molecular oxygen (Halliwell and Gutteridge, 1990), and is the strongest heme binding protein known $\mathrm{K}_{\mathrm{D}} \sim 10^{-13} \mathrm{M}$ (Hrkal et al., 1974). Yet the reasons for the exceedingly tight binding of heme to $\mathrm{Hx}$ are not very well understood from a physical perspective. This is because of a lack of definitive structural investigations of the complex heme-Hx, perhaps because of its large size $>60 \mathrm{KDa}$, and because $\mathrm{Hx}$ is heavily and heterogeneously glycosylated (Noiva et al., 1987), to $\sim 20 \%$ by weight.

Tissue removal of heme implies the presence of a plasma membrane receptor for heme or hemeeprotein complexes in liver cells. This has been a controversial issue. A heme-Hx receptor has been reported to exist in the liver for various species (Smith and Morgan, 1981; Smith et al., 1988). On the other hand, it has been reported that heme bound to Hx may not reach the liver cells as does heme bound to serum albumin (Sinclair 
et al., 1988). Therefore, albumin may be the primary agent for heme delivery to liver cell receptors (Muller-Eberhard and Nikkilä, 1989).

This chapter provides information on the stability of $\mathrm{Hx}$ during the process of isolation in the hope of providing a better understanding the complex heme-Hx. The isolation of $\mathrm{Hx}$ without bound heme has been difficult because from at least some species, it denatures within 24 hours of isolation. However, when heme insertion was performed during isolation of $\mathrm{Hx}$ from plasma, the isolated complex heme $\bullet \mathrm{Hx}$ was very stable. Several interesting characteristics of $\mathrm{Hx}$ were observed and are discussed in this chapter.

\section{MATERIALS AND METHODS}

Hx from human and rat was obtained by affinity chromatography on heme agarose as outlined in Chapter III (Muller-Eberhard, 1988; Tsutsui and Mueller, 1982). Hx from cow, rabbit, human and rat was isolated by a combination of ion exchange and size-exclusion chromatography, as described in Chapter III (Noiva et al., 1987). Electrophoresis (SDS-PAGE), optical spectroscopy, and NMR were performed as explained in Chapters II and III.

\section{RESULTS}

\section{Isolation of Rat ApoHx by Ion Exchange and Gel Exclusion Chromatography}

It was necessary to find a method different from affinity chromatography on heme-agarose, to isolate Hx for NMR studies. This was because the buffer needed to strip the $\mathrm{Hx}$ from the affinity column had a $\mathrm{pH}$ of 2.8 , and our first isolation by this method indicated that the protein may not be stable to such a low $\mathrm{pH}$. $\mathrm{Hx}$ isolation by ion 
exchange chromatography over a CM-sepharose column (Noiva et al., 1987) provided a gentler method for obtaining the protein. This step was reported to separate serum albumin and transferrin from $\mathrm{Hb}$ and $\mathrm{Hx}$, which co-elute (Figure $31 \mathrm{~A}$ ). Gel-exclusion chromatography over Sephadex G-200 was then used to separate Hb from Hx (Figure 31 B). Careful execution of the gel-exclusion chromatography step was very critical because $\mathrm{Hx}$ of molecular weight $\sim 60 \mathrm{KDa}$ is very similar to that of $\mathrm{Hb} \sim 64 \mathrm{KDa}$. Thus a large volume column $(2.5 \times 95 \mathrm{~cm})$, and slow flow rate were used to obtain a clean separation. $\mathrm{OxyHb}$ is a higher molecular weight protein than $\mathrm{Hx}$ and therefore separates before apoHx by gel-exclusion chromatography. Once the $\mathrm{Hx}$ was separated from $\mathrm{Hb}$, heme binding experiments by optical spectroscopy and molecular weight analyses by SDS-PAGE were used to determine which fractions were to be combined and concentrated for NMR studies. Heme binding by $\mathrm{Hx}$ gives a characteristic absorbance spectrum. As in other heme proteins, absorption in the Soret region has a greater intensity (maximum at $412.5 \mathrm{~nm}$ ) than that of the other maxima in the visible region of the spectrum (532.2 and $567.3 \mathrm{~nm}$ ). OxyHb on the other hand, produced a visible spectrum with a Soret maximum at $407.8 \mathrm{~nm}$. The two spectra can be compared in Figure 32.

Within 24 hours of isolating rat $\mathrm{Hx}$, the protein denatured: the protein precipitated, and no heme binding by the supernatant was detected by optical spectroscopy. Denatured Hx was allowed to incubate in a $4 \mathrm{M}$ urea solution for 2 hours which dissolved the precipitate. This solution was then dialyzed against phosphate buffer ( $50 \mathrm{mM} \mathrm{Pi,} 100$ $\mathrm{mM} \mathrm{NaCl}, \mathrm{pH}$ 7.4). Following dialysis, heme binding experiments by optical spectroscopy detected the formation of heme-Hx. SDS-PAGE was used to compare denatured $\mathrm{Hx}$ with urea renatured $\mathrm{Hx}$ (bound to heme) and heme-Hx that was not denatured. The SDS-PAGE bands appeared at the same location (Figure 33). Therefore, the protein remained as a single polypeptide chain throughout this treatment. 
The NMR spectrum of renatured heme.Hx, although not strictly characteristic of an unfolded globular protein, showed no well-resolved hyperfine shifted resonances (Figure $34 \mathrm{~A}$ ), as expected for intact heme•Hx (Figure $34 \mathrm{~B}$ ). Although optical spectroscopy and SDS-PAGE suggested that renatured heme $\cdot \mathrm{Hx}$ (urea treated) was behaving similarly to heme•Hx (not urea treated), NMR spectroscopy proved otherwise. The protein was therefore useless for these studies.

\section{Initial Modification of ApoHx Isolation}

It was obvious after the experiment discussed above that $\mathrm{Hx}$ without bound heme is very unstable when removed from its plasma environment. It was necessary to find a modification that permitted the isolation of a intact Hx. Sephadex G-200 posed a problem due to its slow elution rate ( $2 \mathrm{~mL} /$ hour), which meant exposing apoHx to a low pH environment (pH 5.9 citrate elution buffer) for approximately four days. The isolation technique was modified by replacing the Sephadex G-200 step with Sephacryl S-200. The advantages would be a much faster elution rate ( $14 \mathrm{~mL} /$ hour), therefore, a shorter time that the unstable $\mathrm{Hx}$ would be exposed to the $\mathrm{pH} 5.9$ eluting buffer. This modification resulted in a very poor separation of $\mathrm{Hx}$ from $\mathrm{Hb}$. Assuming that $\mathrm{Hx}$ is more stable at $\mathrm{pH} 7.5$, the eluting buffer was modified from $\mathrm{pH} 5.9$ citrate buffer into $\mathrm{pH}$ 7.5 Phosphate/NaCL buffer. The goal of this modification was to decrease the elution rate in hope of obtaining a cleaner separation of the $\mathrm{Hx}$ from $\mathrm{Hb}$ in a more stable environment. This process was not successful in accomplishing a clean separation, the isolated $\mathrm{Hx}$ was poorly resolved from $\mathrm{Hb}$ resulting in $\mathrm{Hb}$ contamination

\section{Heme.Hx Isolation}

Since $\mathrm{Hx}$ is most stable after the insertion of heme, the procedure was modified such that $\mathrm{Hx}$ would be reconstituted with excess heme after the ion exchange step and prior to 
gel-exclusion chromatography over Sephacryl S-200. After separating serum albumin and transferrin from apoHx and $\mathrm{Hb}$ (Figure $35 \mathrm{~A}$ ), the solution of the latter two was incubated with ten fold excess heme for one hour and adjusted to $\mathrm{pH}$ 7.5. Excess heme was removed over a $(0.5 \times 5 \mathrm{~cm})$ column of DEAE-Sephadex equilibrated with $50 \mathrm{mM}$ Phosphate/100 mM NaCL, pH 7.5. An advantage to this step was the exchange of the protein mixture from the $\mathrm{pH} 5.9$ citrate buffer, to the more stable $\mathrm{pH} 7.5$ Phosphate/NaCL buffer. Since the $\mathrm{Hx}$ at this stage was complexed to heme, the rate of elution over the S-200 gel-exclusion column, equilibrated with $\mathrm{pH} 7.5$ Phosphate/NaCL buffer, was slowed down considerably to $7 \mathrm{~mL} / \mathrm{hr}$ without fear of denaturation of $\mathrm{Hx}$. This modification was very successful, a stable and pure heme-Hx was isolated (Figure $35 \mathrm{~B}$ ). However, a disadvantage of this procedure was the fact that every time a new sample had to be generated, the entire isolation procedure had to be repeated. This procedure was repeated for the isolation of rat, human, and rabbit $\mathrm{Hx}$. It was observed that oxyHb eluted before heme-Hx by gel-exclusion chromatography as seen in Figure 35.

\section{DISCUSSION}

The results in this chapter emphasize the delicate nature of $\mathrm{Hx}$, its instability when in the apo state, and the importance of isolating it under the mildest conditions possible. This is why the heme-agarose technique was not used. Even though optical spectroscopy and SDS-PAGE showed renatured $\mathrm{Hx}$ as an intact protein capable of binding heme, NMR spectroscopy, revealed no sharp, well-resolved hyperfine shifted resonances, indicating that the protein may be heterogeneously folded. This may explain the controversy centering around heme- $\mathrm{Hx}$, and the question of a receptor in the liver. The method of $\mathrm{Hx}$ isolation, its purity, as well as the ratio of heme to $\mathrm{Hx}$ in the sample (Muller-Eberhard, personal communication) may play big roles in its behavior. The isolation protocol 
worked-out here was designed to be careful in maintaining $\mathrm{Hx}$ at $\mathrm{pH} 7.5 \mathrm{in} \mathrm{a}$ phosphate/saline buffer and a heme to $\mathrm{Hx}$ ratio of $1: 1$.

It is evident that $\mathrm{Hx}$ undergoes a conformational changes upon binding to heme. This information may be important for receptor recognition. NMR spectroscopy is the probe that can provide detailed information on the heme-binding pocket of heme-Hx and homogeneity of folding. NMR findings on the heme complex of four species are discussed in Chapter VIII.

\section{ACKNOWLEDGEMENTS}

Special thanks is given to Professor Gordon Kilgour (Portland State University) for his guidance and help with all the protein isolation techniques, and supplying gels and columns for the same purpose. Thanks is also given to Dr. Ursula Muller-Eberhard (Cornell University Medical College) for helpful discussions and supplying the plasma for $\mathrm{Hx}$ isolation. 

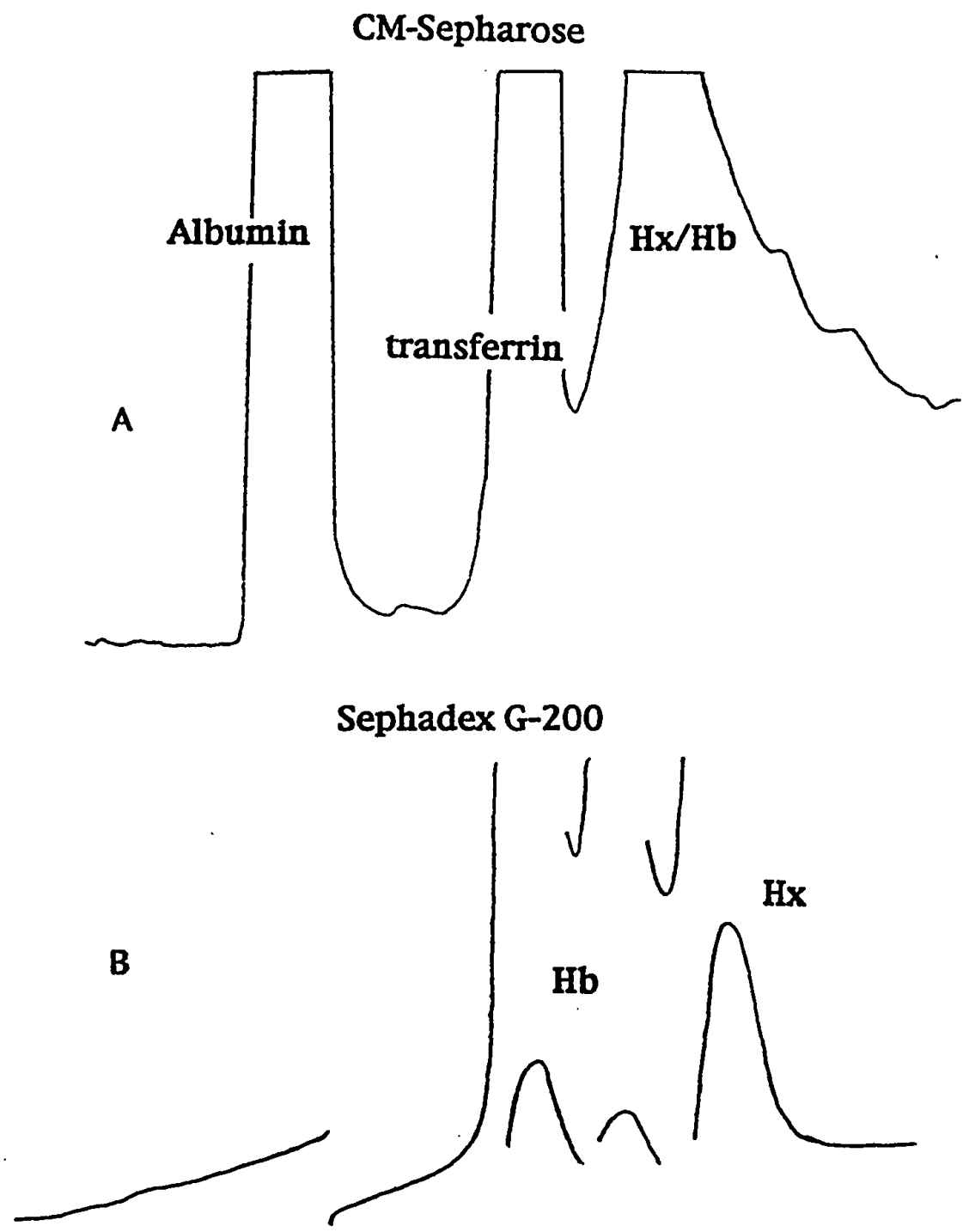

Eigure 31. Chromatography traces for the isolation of Rat Hx. Trace (A) is the chromatogram obtained for the separation of Rat serum albumin from transferrin and (hemoglobin/hemopexin) by ion-exchange over CM-Sepharose at pH 5.9. Trace (B) is the chromatogram obtained by separating hemoglobin from hemopexin over a Sephadex G-200 gel-exclusion column. 


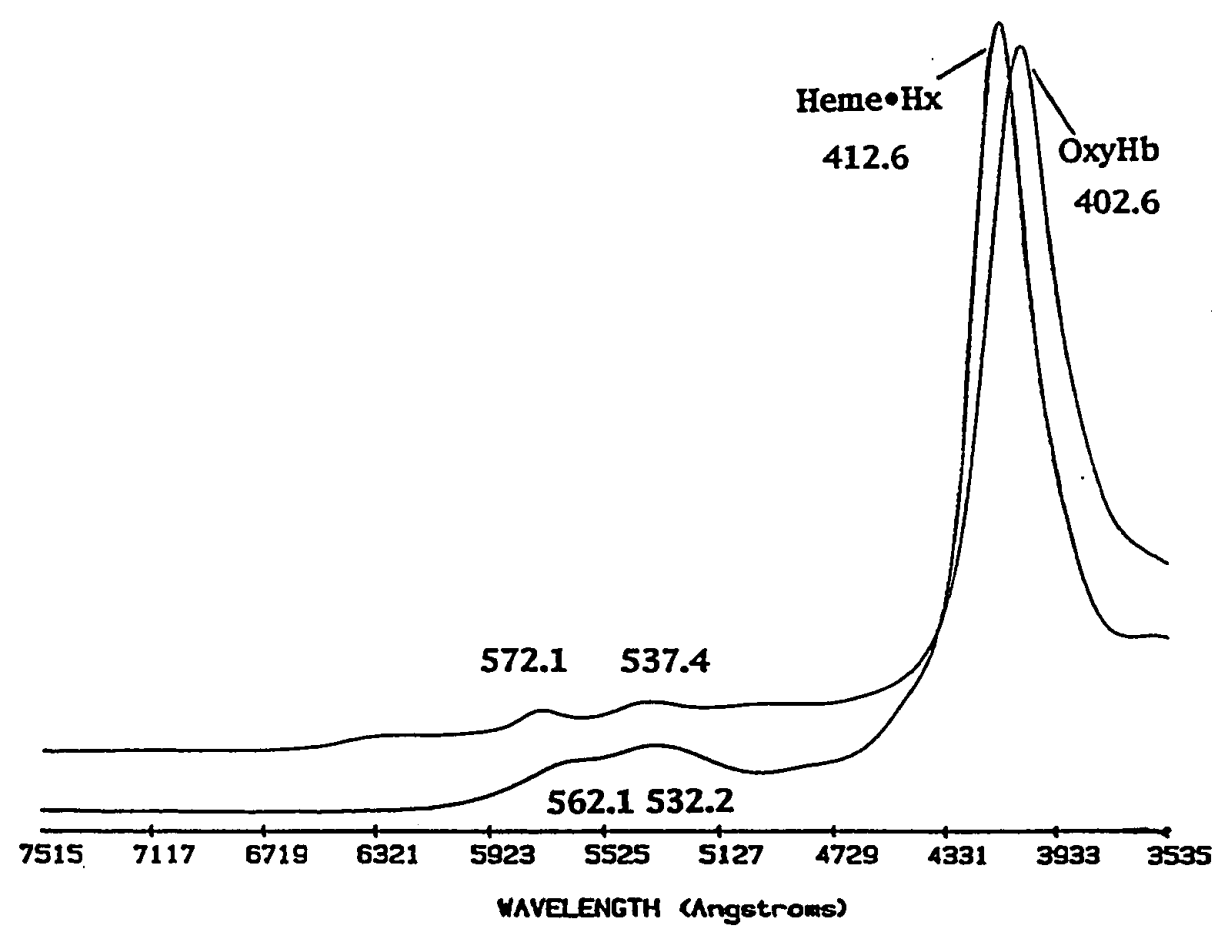

Eigure 32. Optical spectra of Rat oxyhemoglobin (right) and hemopexin (left). Note the characteristic absorption maximum $(412.6 \mathrm{~nm})$ in the Soret region of Rat heme•Hx. 


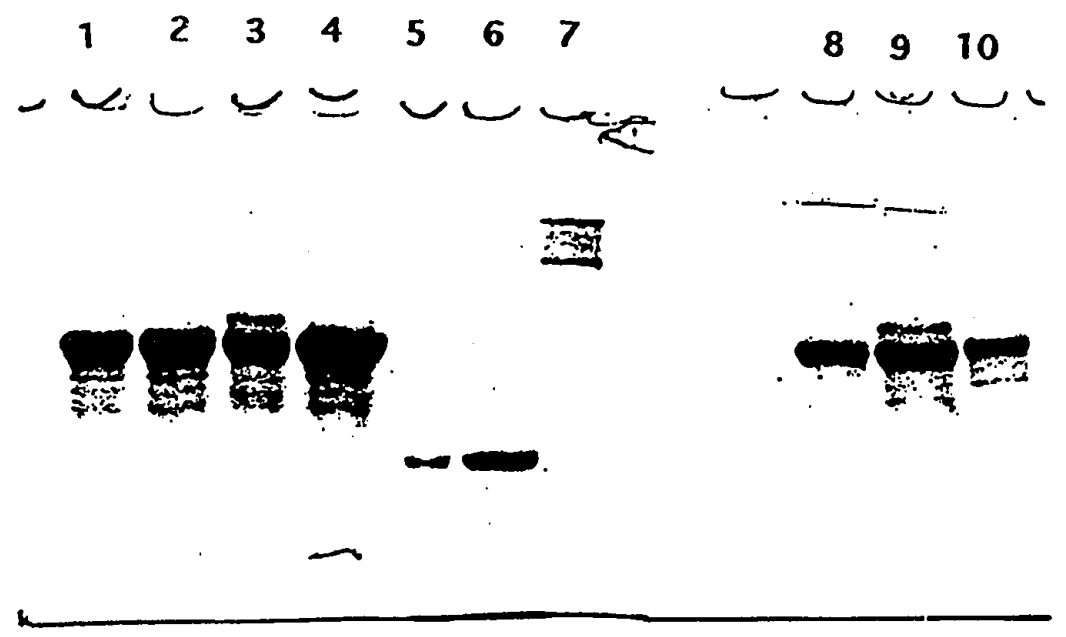

Figure 33. SDS-PAGE gels. Lanes 1, 2, and 4 represent isolated apoHx by ion-exchange prior to denaturation. Lane 10 represents denatured Hx. Lane 8 shows apoHx after urea treatment. Note that lanes 8 and 10 are identical indicating that denatured apoHx was renatured after urea treatment. Lanes 3 and 9 show heme-Hx isolated by the Heme-Agarose method. These lanes show an extra band which is missing from $\mathrm{Hx}$ isolated by the ion-exchange method. Lanes 5,6 , and 7 illustrate how bands show up in various locations indicating variable sized proteins. 


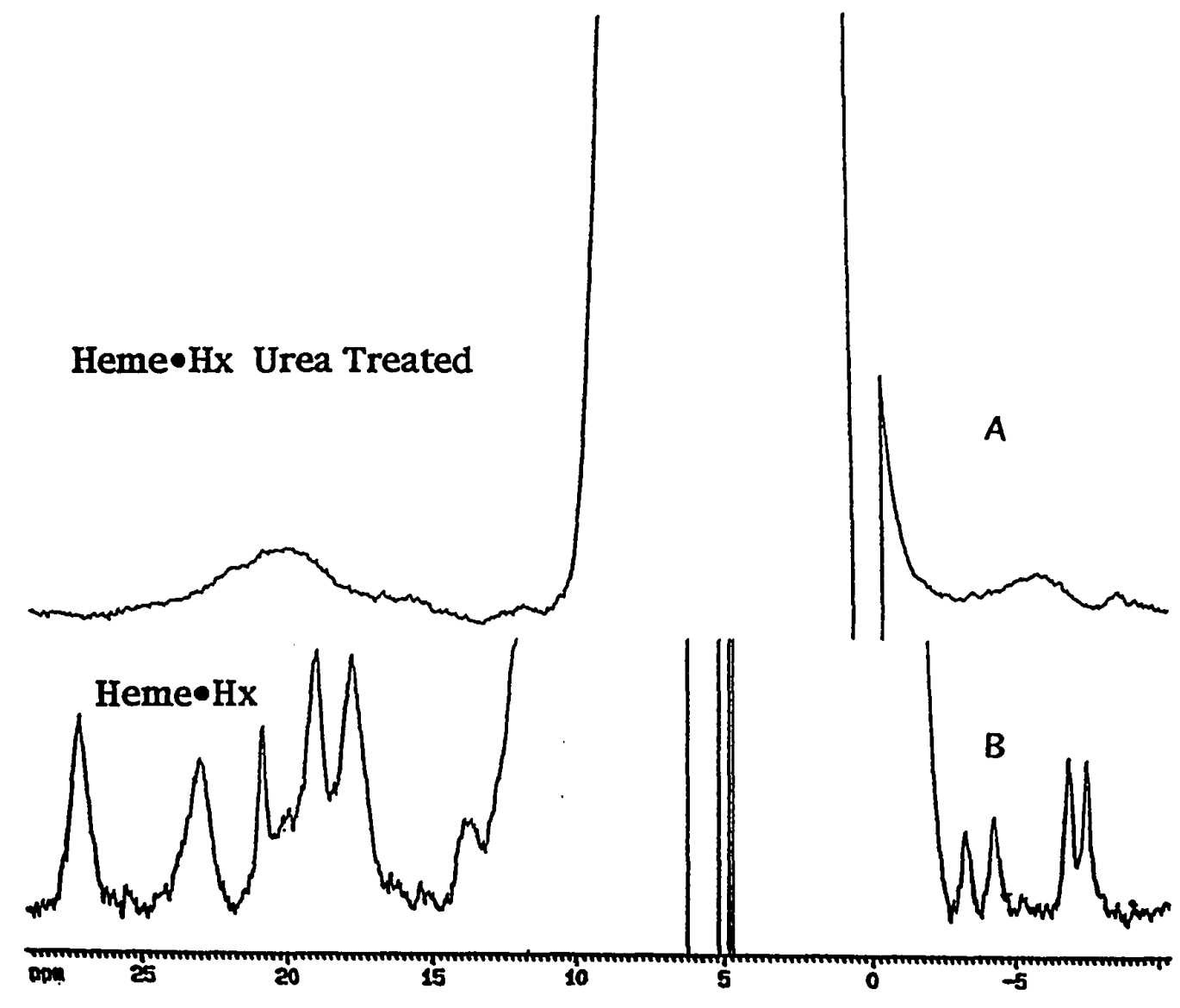

Eigure 34. 1H NMR spectra of Rat heme-Hx. (A) Represents heme-Hx following urea treatment. (B) Shows the spectrum of heme-Hx with no urea treatment. Note that although spectrum $(A)$ shows that heme-Hx is unfolded or heterogeneuosly folded, optical spectroscopy and SDS-PAGE did not give any indication for such an observation. 

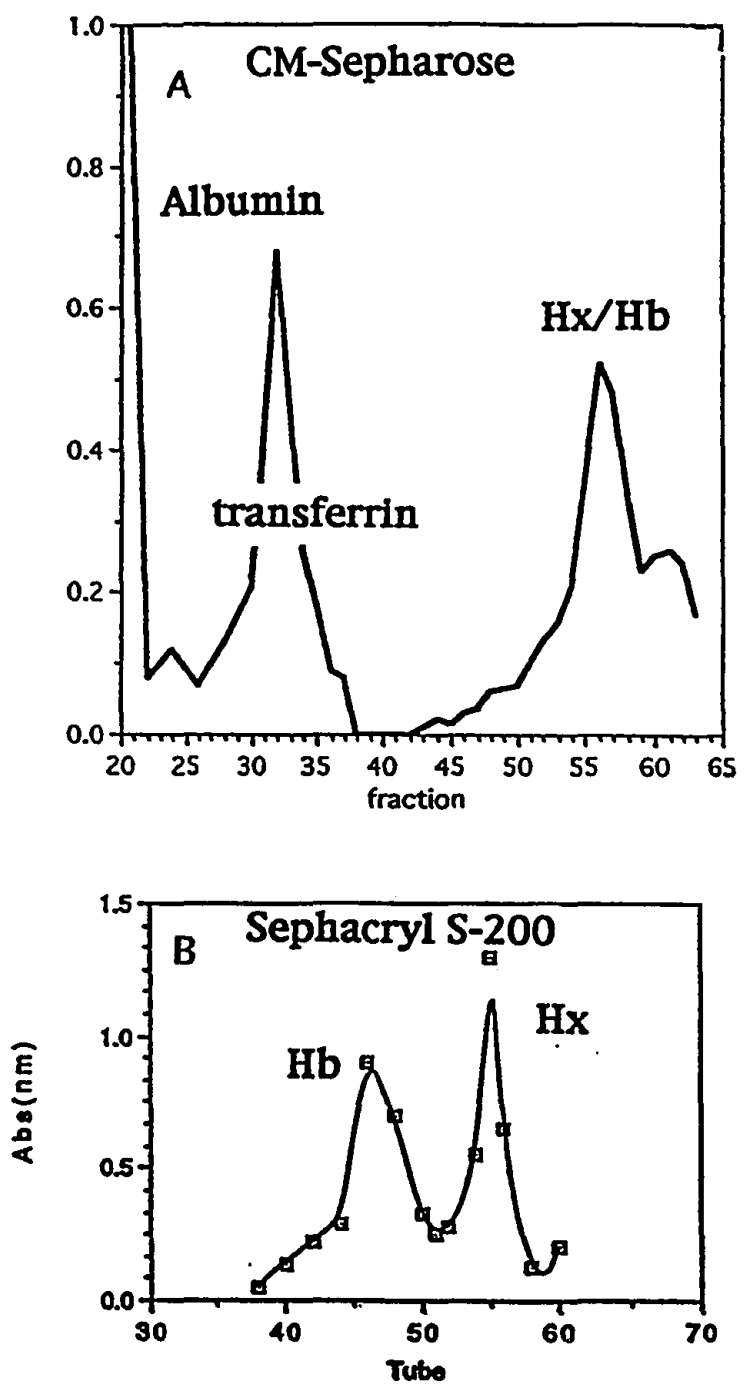

Figure 35. Chromatography traces for the isolation of Rabbit Hx. Trace (A) shows the chromatogram obtained for the separation of Rabbit serum albumin and transferrin from $(\mathrm{Hx} / \mathrm{Hb})$ by ion-exchange over $\mathrm{CM}$-Sepharose at $\mathrm{pH}$ 5.9. Trace (B) shows the chromatogram obtained for the separation of $\mathrm{Hb}$ from $\mathrm{Hx}$ over Sephacryl S-200 gel-exclusion column. 


\section{CHAPTER VIII}

\section{PROTON NMR STUDY OF THE HEME COMPLEX OF HEMOPEXIN}

${ }^{1} \mathrm{H}$ NMR studies of heme proteins in their paramagnetic states have been very useful in defining heme pocket structures and biological behaviors (Satterlee, 1986). The potential of ${ }^{1} \mathrm{H}$ NMR in these systems has been increased dramatically by the application of multi-dimensional methods (Peyton, 1991; Yu et al., 1990), although not yet for a paramagnetic molecule as large as heme $\cdot \mathrm{Hx}$.

The best evidence so far implies that the heme is bound at the surface of $\mathrm{Hx}$, in contrast to what might be expected from the strong inhibition of heme-catalyzed oxidation reactions by $\mathrm{Hx}$. In fact, the heme seems to be quite exposed to solvent (Morgan et al., 1976). Chemical modification (Morgan et al., 1988; Morgan and MullerEberhard, 1976), sequence comparison (Nikkilä et al., 1991), and proteolytic digestion (Muster et al., 1991) data are suggestive of two histidine ligands to the heme iron (Figure $36 \mathrm{~A})$. One of the axial ligands can be replaced, either by $\mathrm{CO}$ when heme $\mathrm{Hx}$ is in the FeII state (Muller-Eberhard and Grizzuti, 1971), or by $\mathrm{CN}^{-}$when heme-Hx is in the Fe III

state (Hrkal et al., 1981). This chapter presents NMR data on the complex heme.Hx, and demonstrates that two-dimensional ${ }^{1}$ H NMR is not only practical, but very useful in making resonance assignments. It is demonstrated unambiguously that this complex is low spin ( $S=1 / 2)$ paramagnetic, resulting from the fact that it has two high-field axial histidine ligands. A species comparison for heme $\mathrm{Hx}$ provides evidence that the protein portion of this complex can exist in various folding states. The material presented in this chapter was performed in collaboration with Dr. Ursula Muller-Eberhard, and the paper: 
Deeb, R.S., Muller-Eberhard, U., and Peyton, D.H. (1993) "Proton NMR Study of the Heme Complex of Hemopexin" is being prepared for publication.

\section{MATERIALS AND METHODS}

Hx from human and rat was obtained by affinity chromatography on heme-agarose as reported in Chapter III (Muller-Eberhard, 1988; Tsutsui and Mueller, 1982). Hx from cow and rabbit was isolated by ion exchange and size-exclusion chromatography as described in Chapter III (Noiva et al., 1987).

\section{RESULTS}

\section{Species Comparison}

Spectra for the heme complex of $\mathrm{Hx}$ from a variety of species (heme complex of Hx human, $\mathrm{Hx}_{\text {cow }}, \mathrm{Hx}_{\text {rat }}$, and $\mathrm{Hx}_{\text {rabbit }}$ are shown in Figure $37 \mathrm{~A}-\mathrm{D}$. It is immediately obvious that the ${ }^{1} \mathrm{H}$ NMR spectrum of heme-Hx is quite species-dependent. This is not evident from optical spectra of the various species which all appear exactly identical to each other (as seen in Figure 32). This is also the case for any other physical method used to date for detecting the heme complex of Hx. The rabbit heme-Hx (Figure $37 \mathrm{D}$ ) has by far the best signal-to-noise ratio, even though not substantially more of this protein was used $(\sim 15-20 \mathrm{mg}$ of $\mathrm{Hx} / 500 \mu \mathrm{L}$ volume). This may reflect a relatively higher stability of the apoHx $x_{\text {rabbit }}$ tertiary structure. In other words, if the protein is unstable in the apo-state, it is possible that upon reconstitution with the prosthetic group, some of the apo-protein remains unfolded and is thus denatured. However, if the apo-protein is stable, it is possible that the majority of it reconstitutes with the prosthetic group, accounting for the more defined and higher signal-to-noise ratio rabbit heme•Hx NMR 
spectrum. Another explanation would be the heterogeneity of the folding of apoHx. It is possible that multiple conformations are obtained during the process of reconstitution with the heme, giving rise to broad and non-descript peaks as seen in the spectra of Figure 37 A-C (compare Figure 34). The precise spectral differences in A-D of Figure 37 are superimposed on the fact that all of the spectra represent low-spin species, with similar hyperfine-shifted peaks, and likely have the same set of iron axial ligands. These ligands are presumed to be from histidine residues and the data confirms this. The inset of Figure $37 \mathrm{D}$ shows that rabbit heme-Hx has two very broad resonances, at -14.5 and at $-22.6 \mathrm{ppm}$. These peaks have very short $\mathrm{T}_{1} \mathrm{~s}(<2 \mathrm{~ms}$; Table $\mathrm{V}$ ) and are very broad (short $T_{2} s$ ). $T_{1}$ and $T_{2}$ are relaxation times such that $T_{1}$ and $T_{2}$ reflect the rate of tumbling of the molecule in question (size and proximity of the histidine ligands to the iron paramagnetic center). By applying the equation for dipolar distance from the paramagnetic center $T_{1} i / T_{1} j^{j}=\left(r_{j} / r_{j}\right)^{6}$, it was possible to approximate the distance between the histidine protons to the Fe center. Taking the distance for the heme $\mathrm{Fe}$ to $\mathrm{CH}_{3}$ as 6.1 $\AA\left(r_{i}\right)$, the methyl $T_{1}=60 \mathrm{~ms}\left(\mathrm{~T}_{1}{ }^{\mathrm{i}}\right)$ (Table $\mathrm{V}$ ), and the His- $\mathrm{H}_{\text {ring }}$ to be $2 \mathrm{~ms}\left(\mathrm{~T}_{1}{ }^{\mathrm{j}}\right)$, the protons giving rise to peaks $\mathrm{a}$ and $\mathrm{b}$ in the inset of Figure $36 \mathrm{D}$ were calculated to approximately reside $<3.5 \AA$ from the Fe. This is close to the SwMb proximal His (F8) distance $\mathrm{r}\left(\mathrm{C}_{\delta, \varepsilon} \mathrm{H}, \mathrm{Fe}\right)=3.4 \AA$. The NMR data is therefore consistent with the proposed bis(histidyl) ligation of the heme iron in at least the rabbit protein.

\section{Cyanide Complex of Rabbit Heme:Hx}

Figure $37 \mathrm{E}$ shows the result of adding cyanide to heme $\mathrm{Hx}$ rabbit. The goal was to observe the changes in the NMR spectrum upon replacing one of the histidine ligands with a cyano ligand. The only peaks visible in the paramagnetic resolved parts of the spectrum are within the chemical shift range and have linewidths that indicate that the cyanide adduct is low spin. There are some small resonances (indicated by arrows) that 
are of similar chemical shift and pattern to the best resolved peaks in heme.Hx $\mathrm{x}_{\text {rabbit }}$. These peaks may be from a small fraction of the protein that was not converted to the cyanide adduct; however the chemical shifts are not identical. This is not sufficient information to tell whether these peaks arise from heme $\mathrm{Hx}$ or from some other species, perhaps a heme-insertion isomer as found in other proteins (La Mar et al., 1981, for heme-insertion isomers in cytochrome $b_{5}$ ).

\section{Competition Studies}

Due to the sensitivity of ${ }^{1} \mathrm{H}$ NMR with regard to the various species (seen in Figure 37), it was the hope to perform competition studies that would permit the determination of which Hx out of the four species discussed earlier has the highest affinity for heme. In this experiment, heme $\cdot \mathrm{Hx}_{\text {species } 1 \text { was mixed with heme-Hx}} \mathrm{H}_{\text {species } 2}$ and the ${ }^{1} \mathrm{H} \mathrm{NMR}$ spectrum of the mixture was monitored. Unfortunately, the rate of heme transfer was so slow that no heme transfer was observed even after several months (no visible changes were seen in the NMR spectrum). It is likely that the rate of heme transfer was significantly slower than the rate of denaturation of apoHx.

\section{Peak Assignments by Isotope-Labeling Experiments}

Figure 38 A-D presents isotope-labeling experiments, performed by adding deuterated hemes to apoHx $x_{\text {rabbit. }}$ Rabbit $\mathrm{Hx}$ was the protein of choice due to the high signal-tonoise ratio attained in a relatively short NMR time ( 3 hours for rabbit heme-Hx compared to 16 hours for rat heme.Hx). The isolation of rabbit $\mathrm{Hx}$ had to be performed four times by ion-exchange techniques described in Chapter III with four various deuterated hemes ( $C D_{3}$ instead of $\mathrm{CH}_{3}$ at position 1 of the heme porphyrin ring). This technique permitted the deletion or attenuation of the methyl peak of choice from the one-dimensional NMR spectrum, upon deuteration. By comparing a heme $\cdot \mathrm{Hx}_{\text {rabbit }}$ spectrum with a 
$1 C D_{3}$ heme.Hx $x_{\text {rabbit, }}$, the diminished peak in the latter arose from the effect of $1 \mathrm{CD}_{3}$, and therefore, can be assigned to the $1 \mathrm{CH}_{3}$ (marked with $\left(^{*}\right)$ in Figure $38 \mathrm{D}$ ). From these results, it was observed that the $1 \mathrm{CH}_{3}, 8 \mathrm{CH}_{3}$, and $3 \mathrm{CH}_{3}$ resonances were resolved from the envelope of protein resonances (Figure 38 A,C and D). No resonance was deleted from the hyperfine-shifted portions of the spectrum from the $5 \mathrm{CD}_{3}$-labeled material (Figure $38 \mathrm{~B}$ ), so the $5 \mathrm{CH}_{3}$ appears to lie within the -2 to $12 \mathrm{ppm}$ region. The precise pattern which is observed for species that have substantial low-spin character is a consequence of the magnetic axes, and this is largely governed by the orientations of the axial ligands, histidines in this case.

\section{NOESY of Heme.Hx $x_{\text {rabbit }}$}

Figure 39 presents the result of a two-dimensional NOESY of the complex heme $\cdot \mathrm{Hx}_{\text {rabbit. }}$ Again this species was chosen because of its relatively narrow resonance lines in the hyperfine-shifted portions of the spectrum. Assigning three of the four methyl protons by isotope-labeling helped establish a starting point in analyzing the two-dimensional spectrum. The $1 \mathrm{CH}_{3}$ (peak E) shows an NOE to $2 \mathrm{H}_{\beta t}$ (peak d) at 19.25 ppm. The spin system of the vinyl groups corroborates this assignment. NOEs are observed between the $2 \mathrm{H}_{\beta t}$ and $2 \mathrm{H}_{\beta c}$ (peak c) at $-7.02 \mathrm{ppm}$ and between the $2 \mathrm{H}_{\alpha}$ (peak C) and $2 \mathrm{H}_{\beta c}$, and $2 \mathrm{H}_{\alpha}$ and $2 \mathrm{H}_{\beta \mathrm{t}}$ at $22.04 \mathrm{ppm}$. The $1 \mathrm{CH}_{3}$ shows NOE connectivities to the $2 \mathrm{H}_{\mathrm{Bc}}$ at $19.25 \mathrm{ppm}$. The $3 \mathrm{CH}_{3}$ (peak $\mathrm{A}$ ) shows a strong $\mathrm{NOE}$ to a single proton resonance at $18.2 \mathrm{ppm}$ (peak F), which may then be the vinyl $4 \mathrm{H}_{\alpha}$. This assignment is far from certain, however. There is not sufficient information from the NOESY spectrum alone to have allowed unambiguous resonance assignments for the methyls nor for further periphery signals. Table $\mathrm{V}$ provides a listing for the porphyrin resonances assigned in heme $\cdot \mathrm{Hx}$. 


\section{DISCUSSION}

The hyperfine-shifted resonances of heme•Hx have properties which indicate that the complex is paramagnetic and low-spin. In particular, the linewidths, chemical shift range, and temperature-dependencies (higher temperatures proved to give the sharpest resonance peaks as seen in Figure 40) of these resonances dictate this conclusion; previous electron paramagnetic resonance (Morgan and Vickery, 1978; Aisen et al., 1974; Bearden et al., 1974) studies are in agreement. Thus, the complex is 6-coordinate with strong-field ligands, consistent with histidine side chains-coordination at both the $5^{\text {th }}$ and $6^{\text {th }} \mathrm{Fe}$ sites. This is just the pattern which has been proposed for heme $\cdot \mathrm{Hx}$ on the basis of a variety of other methods.

Further evidence for this model is found in the presence of the two very broad, upfield resonances in heme $\cdot \mathrm{Hx}_{\text {rabbit }}$. These resonances are extremely relaxed, so that they must arise from residues bound to the iron; only a coordinated residue could approach close enough to the paramagnetic center to produce such strongly relaxed resonances in a low-spin complex. In all axial histidine-ligated cases studied so far, the upfield paramagnetic shift of one histidine ring proton is accompanied by a similar downfield shift of the alternate ring proton (bis(imidazole)heme: Chacko and La Mar, 1982; cytochrome bs: McLachlan et al., 1988; myoglobin cyanide, and mutants: Emerson and La Mar, 1990A,B; Rajarathnam et al., 1992; cytochrome c peroxidase cyanide, and mutants: Satterlee et al., 1990; horseradish peroxidase cyanide: Thanabal et al., 1987). This is because the magnetic axis symmetry of the iron. Therefore, the presence of two such upfield shifted resonances indicates the presence of two ligands, one at the $5^{\text {th }}$, and one at the $6^{\text {th }}$ site. The other two downfield histidine ring protons are not apparent in the data presented in this chapter, however, they may be located under the crowded regions of the spectra, either within the diamagnetic region or even in the 10 to $25 \mathrm{ppm}$ region. 
TABLE V

NMR PARAMETERS FOR RABBIT HEME•HX

\begin{tabular}{|c|c|c|c|c|}
\hline Peak & Assign. & $\delta^{b}(\mathrm{ppm})$ & $\delta_{\mathrm{dia}^{c}}^{\mathrm{c}}(\mathrm{ppm})$ & $\mathrm{T}_{1} \mathrm{~b}(\mathrm{msec})$ \\
\hline A & $3 \mathrm{CH}_{3}$ & 30.12 & -8 & 54 \\
\hline B & $8 \mathrm{CH}_{3}$ & 22.65 & 17 & 55 \\
\hline $\mathrm{C}$ & $2 \mathrm{H}_{\alpha}$ & 22.04 & 8 & 57 \\
\hline D & & 21.71 & 16 & 57 \\
\hline $\mathbf{E}$ & $1 \mathrm{CH}_{3}$ & 19.25 & 14 & 63 \\
\hline $\mathbf{F}$ & & 17.37 & 13 & 47 \\
\hline d & $2 \mathrm{H}_{\beta \mathrm{t}}$ & -6.16 & 12 & 59 \\
\hline c & $2 \mathrm{H}_{\beta c}$ & -7.02 & 15 & 64 \\
\hline b & His- $\mathrm{H}_{\text {ring }}{ }^{d}$ & -14.5 & 13 & $\leq 2^{e}$ \\
\hline a & His- $\mathrm{H}_{\text {ring }}{ }^{d}$ & -22.6 & 19 & $\leq 2^{e}$ \\
\hline
\end{tabular}

aPeaks correspond to designations in Figures 37,38 , and 39. bRecorded $^{\circ} 40^{\circ} \mathrm{C}$ and $\mathrm{pH}$ 7.0. CFrom extrapolations to $\mathrm{T}^{-1}=0$ (Curie plot). dTentative assignment. eEstimated: $T_{1}$ too short for accurate determination under conditions used.

The ligation pattern with two histidines occupying both the $5^{\text {th }}$ and $6^{\text {th }} \mathrm{Fe}$ site, may help to explain the ability of $\mathrm{Hx}$ to inhibit heme-catalyzed oxidations. These oxidations are inhibited when oxygen cannot reach the iron center. Access to the heme by oxygen may be blocked by either the two strongly ligated axial histidines (discussed above), or by the heme being buried deep within the protein matrix. However, as mentioned earlier in this chapter, studies have indicated that the heme is solvent exposed. When the iron is in the ferric state, the axial ligands are very difficult to displace. Therefore, the data discussed in this chapter indicates that the remarkable feature of $\mathrm{Hx}$ as an antioxidant is primarily due to the nature of the axial ligation involved with heme binding. 


\section{ACKNOWLEDGEMENTS}

Thanks is given to Professor Kevin Smith (University of California at Davis) for supplying the isotope-labeled hemes. 

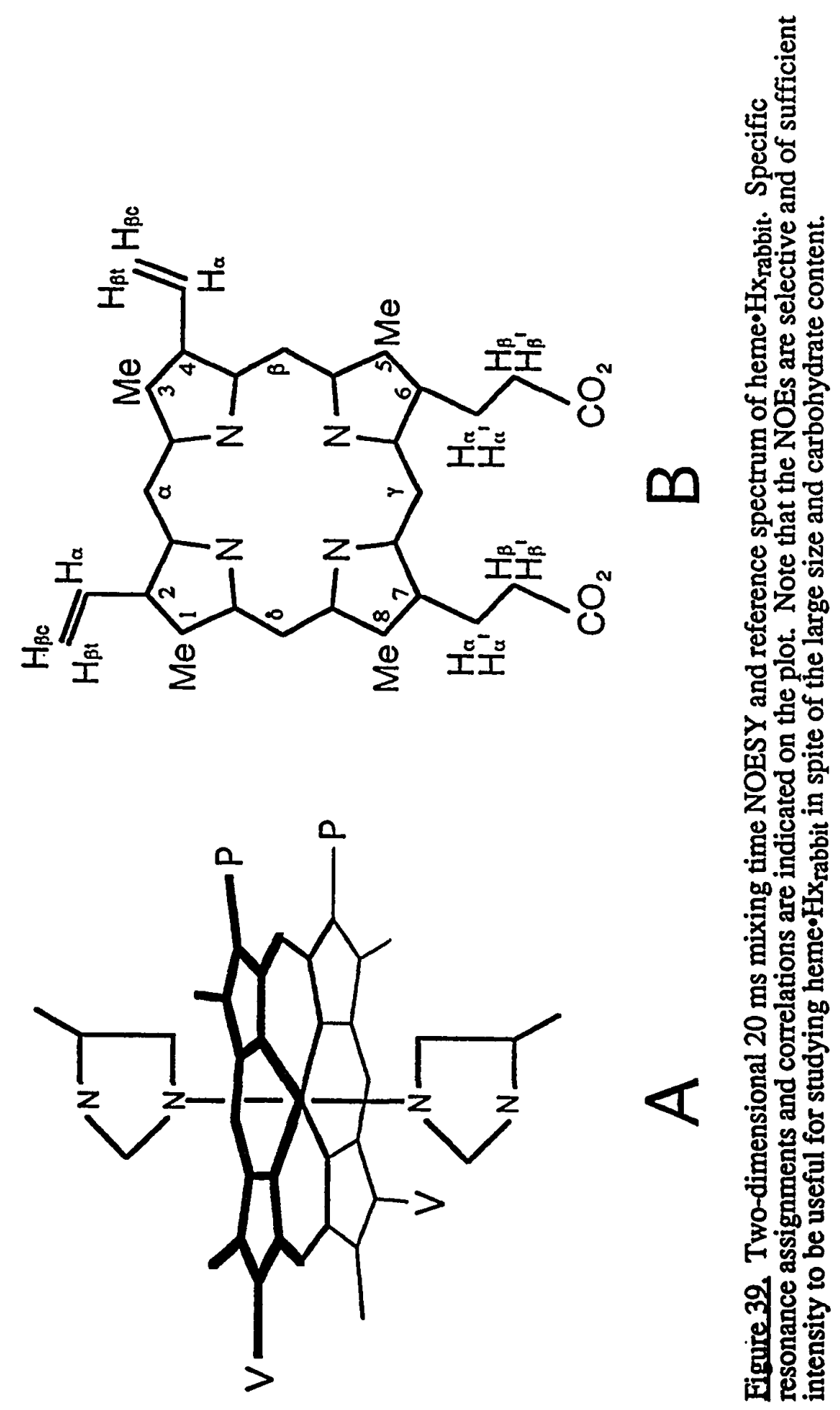

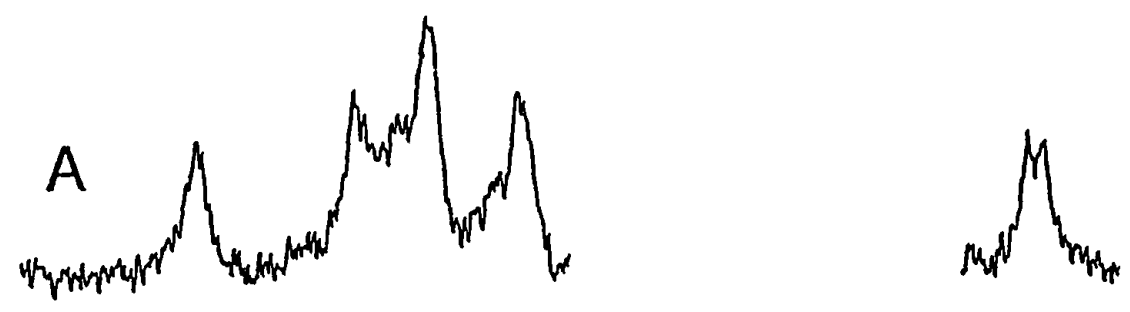

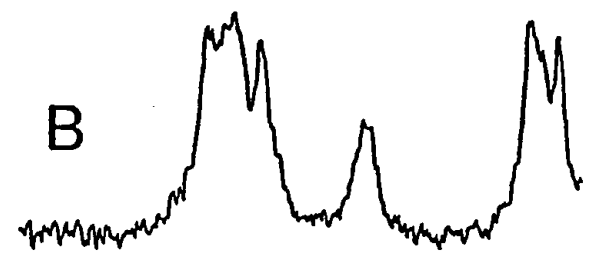
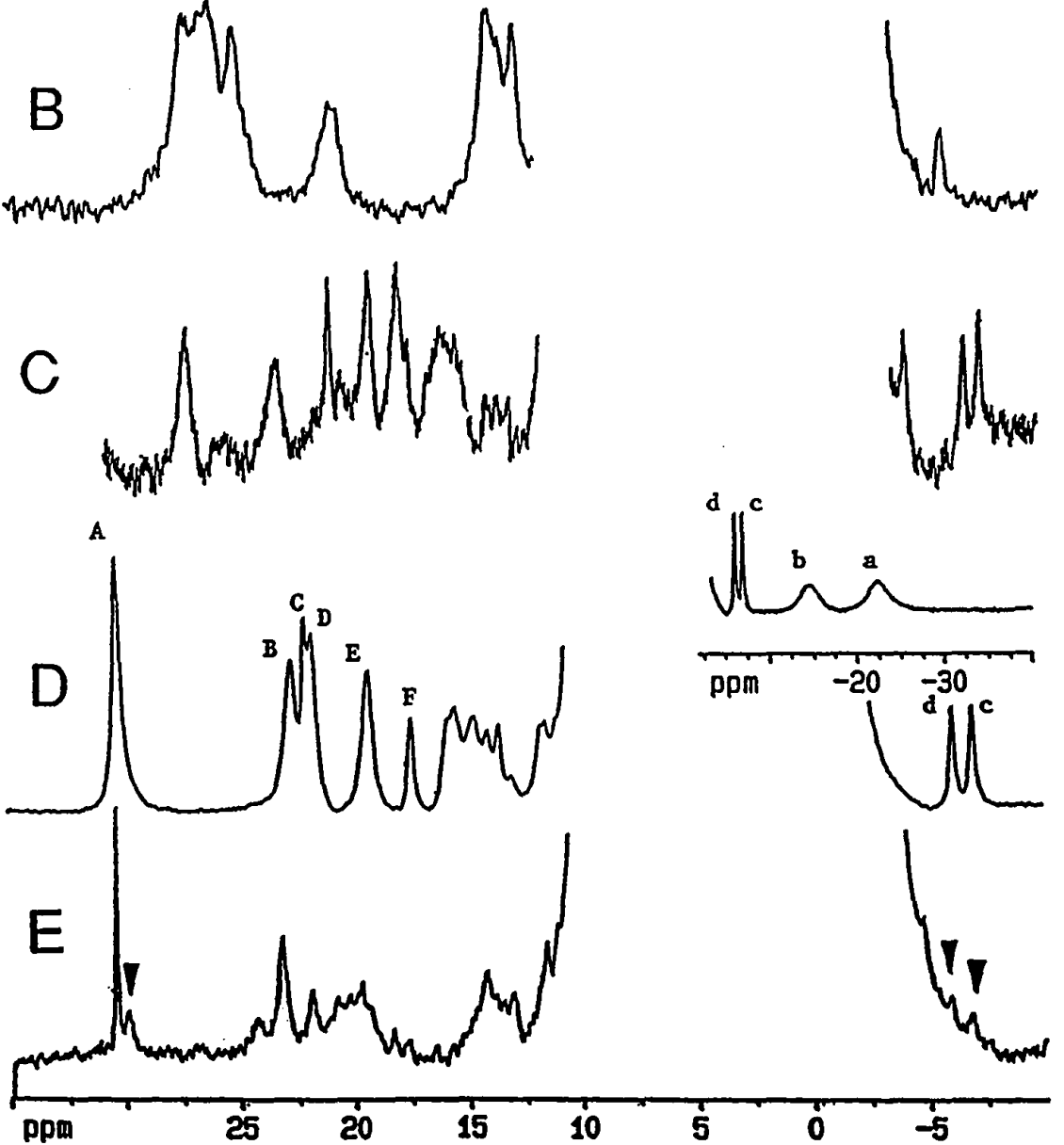

Eiqure 37. Species comparison for the hyperfine-shifted portions of the ${ }^{1} \mathrm{H}$ NMR spectrum of heme $\mathrm{Hx}$. (A) Heme-Hx human. (B) Heme.Hx $\mathrm{H}_{\text {cow. }}$ (C)

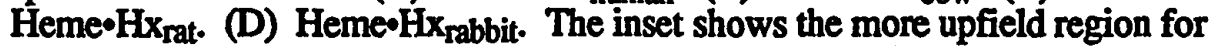
heme- $\mathrm{Hx}$ rabbit. Note that the rabbit spectrum is of much higher quality than from the other species. (E) HemeoH $x_{\text {rabbit }} \mathrm{CN}$. The peaks marked with arrows may arise from heme $\mathrm{Hx}_{\text {rabbit, }}$ although they do not have exactly the expected chemical shifts. 


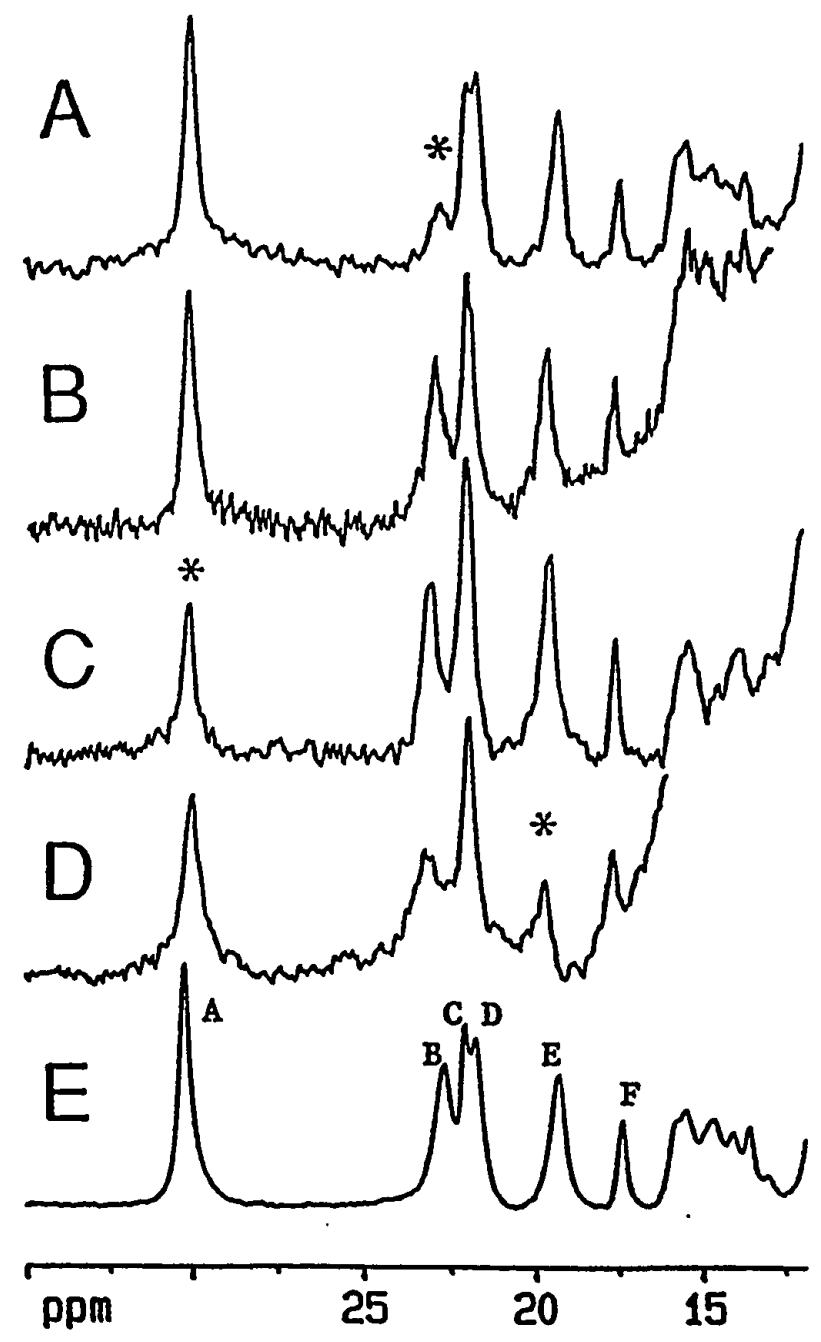

Eigure 38. ${ }^{1} \mathrm{H}$ NMR spectra of heme-Hx$x_{\text {rabbit }}$ generated using isotopically-labeled hemin. (A) $8 \mathrm{CD}_{3}$-heme-Hx. (B) $5 \mathrm{CD}_{3}$-heme $\mathrm{Hx}$. (C) $3 \mathrm{CD}_{3}$-hemeoHx. (D) $1 \mathrm{CD}_{3}$-heme $\mathrm{Hx}$. (E) HemeoHx. Note that although these spectra allow assignment of resonances, there is residual intensity for each peak. This might have resulted from some fraction of the $\mathrm{Hx}$ isolated from the serum being already complexed with heme, or from the presence of a heme-insertion isomer. 


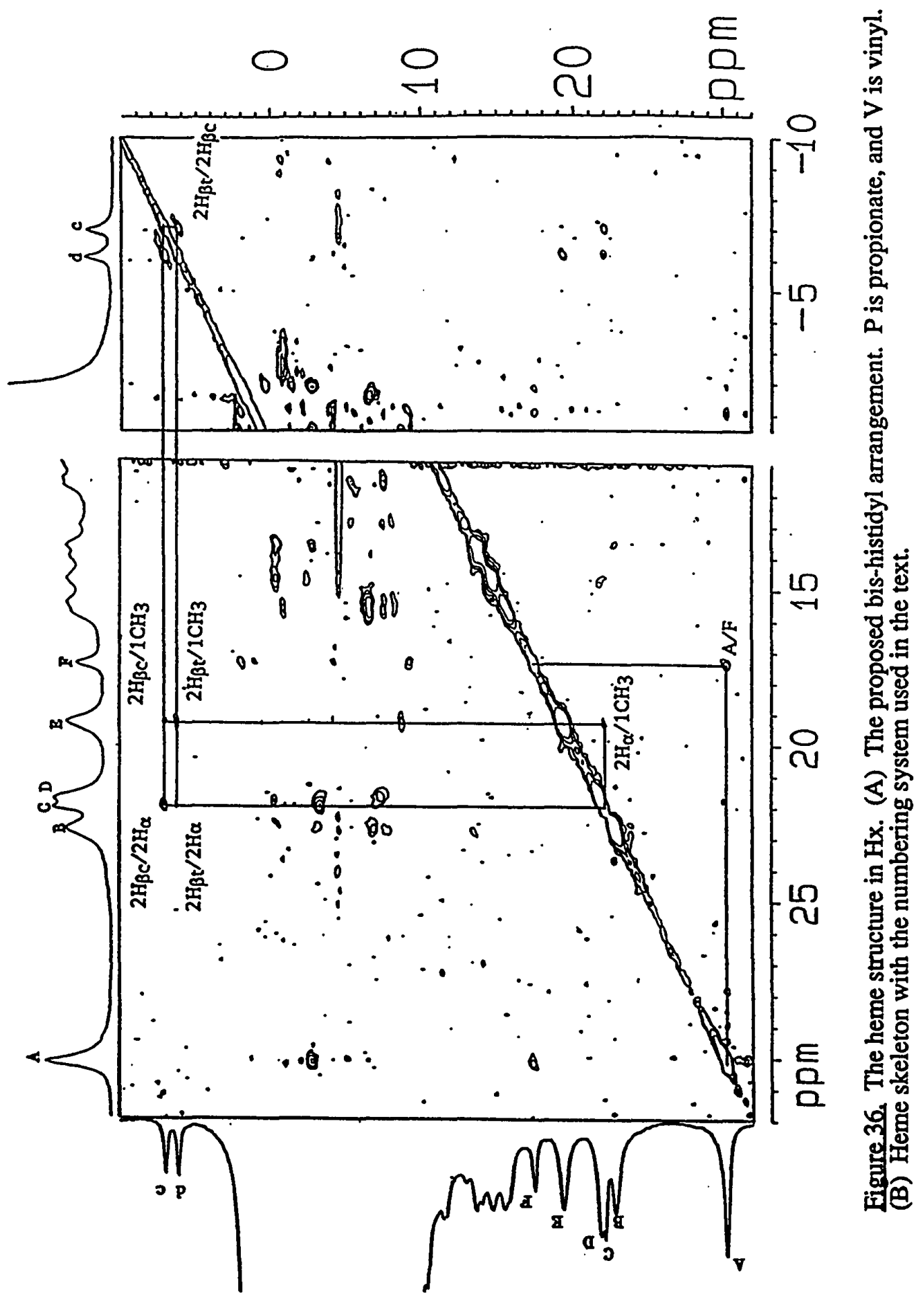




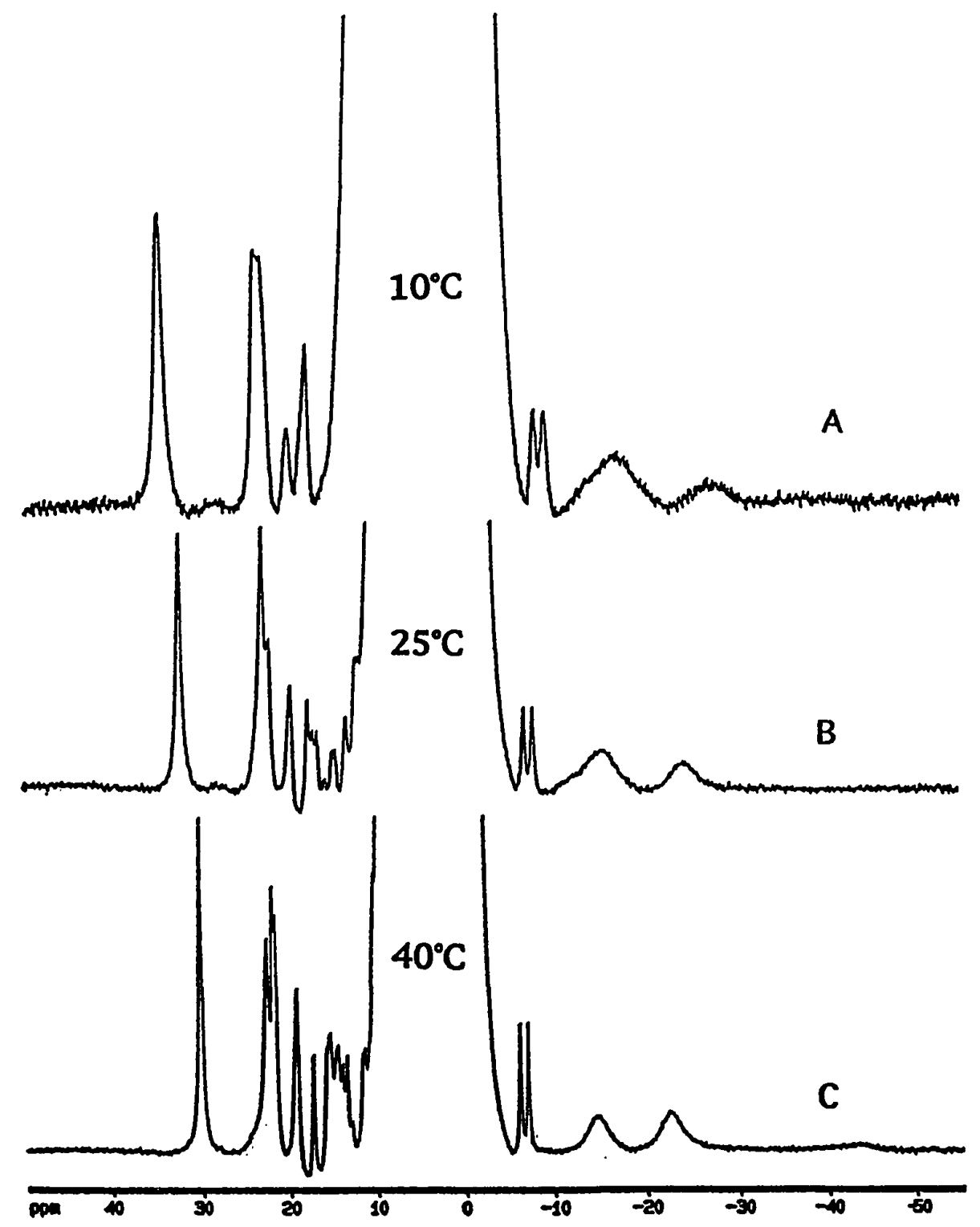

Eigure 40. '1H NMR spectra of heme-Hx dependence. (A) $10^{\circ}$, (B) $25^{\circ}$ and (C) $40^{\circ} \mathrm{C}$. Note that the resolution for heme- $\mathrm{Hx}_{\text {rabbit }}$ at $40^{\circ} \mathrm{C}$ is significantly improved from the spectra obtained at lower temperatures. 


\section{CHAPTER IX}

\section{CONCLUSION}

At the time this dissertation research was started, SnPP was under clinical investigation for its therapeutic role in inhibition of heme-oxygenase, and $\mathrm{Hx}$ was being investigated for its role as an antioxidant and therefore a potential drug for the treatment of porphyria and other circulatory diseases. Therefore, a need to investigate the effects of SnPP on the structure and therefore the function of some important proteins in a living system including $\mathrm{Hx}$, were in order. $\mathrm{Mb}$ (a well-studied heme-binding protein from muscle tissue) and $\mathrm{Hx}$ were the two proteins of choice. Mb served as a model in order to better understand the binding of SnPP to heme proteins as well as the conformational shifts associated with it. The study of the interaction of Hx with heme followed. NMR has never been performed on $\mathrm{Hx}$, therefore, it was necessary to begin the $\mathrm{Hx}$ reconstitutions with the more physiologically relevant prosthetic group (heme).

Chapters II and III discussed in detail the spectroscopic techniques as well as the protein isolation methods used throughout the investigations discussed in this dissertation. It is important to mention that a great deal of time was spent trying to find an ideal ion-exchange protocol for the isolation of $\mathrm{Hx}$ from plasma. Doing so was very necessary for obtaining a good quality heme-Hx sample that could be studied by NMR.

Chapter IV was dedicated to understanding the structure and behavior of SnPP in solution at various $\mathrm{pH}$ values. Kinetics studies from optical spectroscopy supported the evidence presented by Breslow et al. (1986) that SnPP behaves in solution as a slowly interconverting monomer-dimer system which is $\mathrm{pH}$ dependent. At neutral $\mathrm{pH}$, the dominant form is a dimer, whereas at high $\mathrm{pH}$ values, the dominant form is a monomer. 
At neutral $\mathrm{pH}$ values, ${ }^{1} \mathrm{H}$ NMR, ${ }^{119} \mathrm{Sn}$ NMR and IR spectroscopy show evidence that dimeric ( $\mathrm{SnPP})_{2}$ has a structure consistent with a $\mu$-oxygen bridged oligomer. This was supported by the varying meso-proton chemical shifts observed upon going from monomer to dimer. This conclusion is tentative until $(\mathrm{SnPP})_{2}$ or a related molecule is isolated and characterized more fully.

In Chapter V, it was shown from the ${ }^{1} \mathrm{H}$ NMR assignments of SnPP•EqMb that SnPP inserts into the globin pocket such that a single insertion isomer is detected at equilibrium, similarly to EqMbCO and SwMbCO. The similarities between $\mathrm{MbCO}$ and SnPP•EqMb were striking, indicating very little deformation in the heme-binding pocket. Exceptions were seen with the distal His(64)E7, distal Phe(43)CD1, distal Val(68)E11, and proximal His(97)FG3. The $\varepsilon \mathrm{H}$ of His(64)E7, $\varepsilon \mathrm{H}$ and $\zeta \mathrm{H}$ of Phe(43)CD1 and $\gamma_{\mathrm{CH}}$ of $\mathrm{Val}(68) \mathrm{E} 11$ were all shifted upfield relative to SwMbCO and EqMbCO. This indicated that the side chains of these amino acid residues may be in closer proximity to the distal side of the porphyrin ring in comparison to the species mentioned above. The same conclusion applies to proximal His(97)FG3. ${ }^{119} \mathrm{Sn}$ NMR and ${ }^{1} \mathrm{H}$ NMR pH titrations made it possible to establish the identity of the $5^{\text {th }}$ and $6^{\text {th }}$ ligands to $\mathrm{Sn}$ from the proximal and distal side. ${ }^{1} \mathrm{H}$-Coupling was observed for $\mathrm{Sn}$ in SnPP•EqMb, this arose from the scalar coupling to the protons of the amino acid in question and not from the porphyrin peripheral protons, or from the water ligand. This was confirmed because ${ }^{119} \mathrm{Sn}$ NMR of SnPP alone in solution, showed no coupling (discussed in Chapter IV), and all solutions were exchanged in ${ }^{2} \mathrm{H}_{2} \mathrm{O}$. This made it possible to assume that the $5^{\text {th }}$ ligand of Sn on the proximal side is occupied by an amino acid, His(93)F8 similarly to $\mathrm{SwMbCO}$ and EqMbCO. The $\mathrm{pH}$ titration from 7 to 10 of the meso Hs showed strongly perturbed chemical shifts such that the $\mathrm{pK}_{\mathrm{a}}$ affecting each meso proton was 9.8. This made it possible to assign the $6^{\text {th }}$ ligand on the distal side to water at $\mathrm{pH} 7$, being titrated to $\mathrm{OH}^{-}$at higher $\mathrm{pH}$ values. 
In Chapter VI it was shown that relatively long-lived intermediates within the reconstitution processes:

$$
\mathrm{SnPP}+\text { apoEqMb } \rightarrow \text { SnPP•EqMb* } \rightarrow \text { SnPP•EqMb and }
$$

$$
(\mathrm{SnPP})_{2}+\text { apoEqMb } \rightarrow(\mathrm{SnPP})_{2} \cdot \mathrm{EqMb} \rightarrow \mathrm{SnPP} \cdot \mathrm{EqMb} \text { were attainable and }
$$
comparable to the equilibrium structure SnPP•EqMb. It was found that SnPP•EqMb* had a distal side porphyrin-binding pocket in the vicinity of $\mathrm{Val}(68) \mathrm{E} 11$ virtually identical to $\mathrm{SnPP} \cdot \mathrm{EqMb}$. Val(68)E11 upfield resonances were identical in both species. However, the proximal His(93)F8-Sn bond was declared missing since the estimated rate for SnPP dissociation from SnPP•EqMb* ${ }^{*} K_{\text {off }} \sim 1 s^{-1}$, was very low. This supported the idea that porphyrin reorientation did not require complete dissociation from the globin. Also, His(97)FG3 did not show its resonance at the usual chemical shift indicating that the 7propionate-His(97)FG3 bridge may be missing or perturbed from its usual location in SnPP•EqMb*. It is likely that the F-helix containing His(97)FG3 and His(93)F8 is perturbed from its native position.

Due to the large size of the dimer and tight fit of the globin pocket in the native state, it seemed very surprising that ( $\mathrm{SnPP})_{2}$ can insert into the globin pocket (Chapter VI). However, studies on apoMb have shown that it has reduced helical content relative to the holoprotein and that the F-helix is partly or largely unfolded (Breslow et al., 1965; Harrison and blout, 1990; Hughson et al/, 1990). This structural perturbation in the apo-pocket can provide room for the wide dimer. Another species was observed during the reconstitution of $(\mathrm{SnPP})_{2}$ with apoMb, $\mathrm{SnPP} \cdot \mathrm{EqMb}$. This species however was always minor, and it was not possible to further investigate it. However, the fact that it was only observed at neutral $\mathrm{pH}$ suggested that this species may have been the alternate insertion isomer, since the water ligand can be replaced (as opposed to $\mathrm{OH}$ ) by His(93)F8 quickly enough to trap both insertion isomers, after which only one insertion isomer is observed at equilibrium. 
At this stage of the research, it was decided to pursue the study of another very important heme-binding protein, $\mathrm{Hx}$, with analogous methods to those applied to SnPP.EqMb. No NMR or X-ray crystal work has been achieved for this intact protein. This is due to its carbohydrate heterogeneity and large size (60 $\mathrm{KDa}$ for $\mathrm{Hx}$ compared to $18 \mathrm{KDa}$ for $\mathrm{Mb}$ ). The absence of structural information on this protein, made it necessary to begin its study by interacting it with the well known, and more physiologically relevant heme rather than diamagnetic SnPP. The lack of structural information was not the only difficulty encountered. It was shown in Chapters III and VII the complexity of isolation of $\mathrm{Hx}$ from plasma, and the instability of the protein in the unbound state. It was shown that although all heme-Hx may appear completely folded by techniques such as optical spectroscopy and SDS-PAGE, that was not the case observed by NMR spectroscopy. A sample that appeared to be intact by the other methods, showed no sharp, well-resolved hyperfine shifted resonances, indicating that the protein may be heterogeneuosly folded.

In Chapter VIII, it was shown that NMR, although difficult to accomplish with heme $\cdot \mathrm{Hx}$, is indeed possible, and can provide very important structural information. The complex was shown to be 6-coordinate, paramagnetic and low spin, with histidines occupying the $5^{\text {th }}$ and $6^{\text {th }}$ sites, although some marked variations were observed among the four species discussed. It was possible to observe porphyrin resonances within the heme pocket of the intact rabbit protein by two-dimensional spectroscopy.

The data and conclusions presented in this dissertation provide a very strong and positive backbone for the pursuit of the study of the interactions of $\mathrm{Hx}$ with heme and SnPP. Since NMR observation is possible on a large intact heme $\mathrm{Hx}$, it will be time well spent in the future, to deglycosylate and fragment the protein in order to achieve a smaller size, less heterogeneous complex, easier to observe by NMR. Studying separate protein domains, or a deglycosylated complex by NMR can provide very enlightening structural 
information and may finally provide the definitive answer to the question: Is there a receptor for heme•Hx in the liver? 


\section{REFERENCES}

Aisen, P., Leibman, A., and Harris, D.C. (1974) J. Biol. Chem. 49, 6824-6827.

Abraham, R.J., and Smith, K.M. (1983) J. Am. Chem. Soc. 105, 5734-5741.

Abraham, R.J., Eivazi, F., Pearson, H., and Smith, K.M. (1976) J. Chem. Soc., Chem. Commun. 699-701.

Aft, R.L., and Mueller, G.C. (1984) J. Biol. Chem. 259, 301-305.

Aft, R.L., and Mueller, G.C. (1985) Life Sci. 36, 2153-2161.

Bax, A. (1983) J. Magn. Reson. 53, 517-520.

Bax, A., and Davis, D.G. (1985) J. Magn. Reson. 63, 207-213.

Bax, A., and Davis, D.G. (1985) J. Magn. Reson. 65, 355-360.

Bearden, A., Morgan, W.T., and Muller-Eberhard, U. (1974) Biochem. Biophys. Res. Commun. 61, 265-272.

Bertini, I., and Luchinat, C. (1986) NMR of Paramagnetic Molecules in Biological Systems, Benjamin Cummings, Menlo Park.

Bessis, M.D., and Jensen, W.N. (1965) Br. J. Haematol. 11, 49-51.

Bothner-By, A. A., Stephens, R.L., Lee, J., Warren, C.D., and Jeanloz, R.W. (1984) J. Am. Chem. Soc. 106, 811-813.

Braunshweiler, L., and Ernst, R.R. (1983) J. Magn. Reson. 53, 521-528.

Braunshweiler, L., Bodenhausen, G., and Ernst, R.R. (1983) Mol. Phys. 48, 535-560.

Breslow, E., Beychok, S., Hardman, K.D., and Gurd, F.R.N. (1965) J. Biol. Chem. 240, 304-309.

Breslow, E., Chandra, R., and Kappas, A. (1986) J. Biol. Chem. 261, 3135-3141.

Brown, S. B., Jones, P., and Lantzke, I.R. (1969) Nature 223, 960-961.

Buchler, J.W., Puppe, L., Rohbock, K., and Schneehage, H.H. (1973) Ann. N.Y. Acad. Sci. 206, 116-137. 
Caughey, W.S. (1980) in Advances in Inorganic Biochemistry (Darnell, D.W., and Wilkins, R.G., Eds.) Vol. II, pp 95-115, Elsevier, Amsterdam.

Chacko, V.P., and La Mar, G.N. (1982) J. Am. Chem. Soc. 104, 7002-7007.

Cocco, M.J., and Lecomte, J.T.J. (1990) Biochemistry 29, 11067-11072.

Cooke, R.M., and Wright, P.E. (1987) Eur. J. Biochem. 166, 409-414.

Cowan, J.A., and Gray, H.B. (1989) Inorg. Chem. 28, 4554-4556.

Cremlyn, R. (1978) "Pesticides. Preparation and Mode of Action" John Wiley and Sons, New York.

Cutnell, J.D., La Mar, G.N., and Kong, S.B. (1981) J. Am. Chem. Soc. 103, 3567-3572.

Dalvit, C., and Wright, P.E. (1987) J. Mol. Biol. 194, 313-327.

Dayhoff, M.O. (1972) Atlas of Protein Sequence and Structure. Nat. Biomed. Res. Found., Washington, D.C.

Deeb, R.S., and Peyton, D.H. (1991) J. Biol. Chem. 266, 3728-3733.

Deeb, R.S., and Peyton, D.H. (1992) Biochemistry 31, 468-474.

Dickerson, R.E., and Geiss, I. (1983) Hemiglobins; Structure, Function, Evolution, Pathology, Benjamin-Cummings, Menlo Park, CA.

DiFeo, T.J., and Addison, A.W. (1991) Inorg. Chem. 30, 1151-1153.

Drummond, G.S., and Kappas, A. (1981) Proc. Natl. Acad. Sci., USA 78, 6466-6470.

Drummond, G.S., and Kappas, A. (1982) Science 217, 1250-1252.

Drummond, G.S., and Kappas, A. (1984) J. Clin. Invest. 74, 142-149.

Emerson, S.D., and La Mar, G.N. (1990A) Biochemistry 29, 1545-1556.

Emerson, S.D., and La Mar, G.N. (1990B) Biochemistry 29, 1556-1566.

Evans, S.V., and Brayer, G.D. (1988) J. Biol. Chem. 263, 4263-4268.

Gebe, J.A., Peyton, D.H., and Peyton, J.A. (1989) Biochem. Biophys. Res. Commun. $161,290-294$.

Griko, Y.V., Privalov, P.L., Venyaminov, S.Y., and Kutyshenko, V.P. (1988) J. Mol. Biol. 202, 127-138.

Guiles, R.D., Bassus, V.J., Kuntz, I.D. and Waskell, L. (1992) Biochemistry 31, 11365-11375.

Halliwell, B., and Gutteridge, J.M.C. (1986) Arch. Biochem. Biophys. 246, 501-514. 
Halliwell, B. (1987) FASEB J. 1, 358-364.

Halliwell, B., and Gutteridge, J.M.C. (1990) Arch. Biochem. Biophys. 280, 1-8.

Han, K.-H., La Mar, G.N., and Nagai, K. (1989) Biochemistry 28, 2169-2178.

Harris, R.K., Kennedy, J.D., and McFarlane, W. (1978) NMR and the Periodic Table, pp 342-366, Academic Press, London.

Harrison, S.C., and Blout, E.R. (1965) J. Biol. Chem. 240, 299-303.

Hauksson, J.B., La Mar, G.N., Pandey, R.K., Rezzano, I.N., and Smith, K.M. (1990) J. Am. Chem. Soc. 112, 6198-6205.

Hayes, W. J. (1982) Pesticides Studied in Man. Williams and Wilkins, Baltimore.

Heiny, M.E., Takahashi, N., Noda, S., and Putnam, F.W. (1986) Protides. biol. Fluids 34, 431-436.

Hermes-Lima, M., Valle, V.G.R., Vercesi, A.E. and Bechara, E.J.H. (1991) Biochim. Biophys. Acta 1056, 57-63.

Hoffrnan, A.B., Collins, D.M., Day, V.W., Fleischer, E.B., Srivastava, T.S., and Hoard, J.L. (1972) J. Am. Chem. Soc. 94, 3620-3626.

Hrkal, Z., Vodrazka, Z., and Kalousek, I. (1974) Eur. J. Biochem. 43, 73-78.

Hrkal, Z., Kalousek, I. and Vodrazka, Z. (1981) Studia Biophysica 82, 69-73.

Hughson, F.M., Wright, P.E., and Baldwin, R.L. (1990) Science 249, 1544-1548.

Hunter, C.A., and Sanders, J.K.M. (1990) J. Am. Chem. Soc. 112, 5525-5534.

Johnson, C.E., and Bovey, F.A. (1958) J. Chem. Phys. 29, 1012-1014.

Jue, T., Krishnamoorthi, R., and La Mar, G.N. (1983) J. Am. Chem. Soc. 105, 57015703.

Kawamura-Konishi, Y., Kihara, H., and Suzuki, H. (1988) Eur. J. Biochem. 170, 589-595.

Kirschner-Zilber, I., Rabizadeh, E., and Shaklai, N. (1982) Biochim. Biophys. Acta 690, 20-30.

Kumar, A., Ernst, R.R., and Wuthrich, K. (1980) Biochem. Biophys. Res. Commun. 95, $1-6$.

Kuriyan, J., Wilz, S., Karplus, M., and Petsko, G.A. (1986) J. Mol, Biol. 192, 133-154.

La Mar, G.N., Eaton, G.R., Holm, R.H., and Walker, F.A. (1973) J. Am. Chem. Soc. 95, 63-75. 
La Mar, G.N. (1979) in Biological Applications of Magnetic Resonance (Shulman, R.G., ed) pp. 305-343, Academic Press, New York.

La Mar, G.N., Burns, P.D., Jackson, J.T., Smith, K.M., Langry, K.C. and Strittmatter, P. (1981) J. Biol. Chem. 256, 6075-6079.

La Mar, G.N., Davis, N.L., Parish, D.W., and Smith, K.M. (1983) J. Mol. Biol. 168, 887-896.

La Mar, G.N., Toi, H., and Krishnamoorthi, R. (1984) J. Am. Chem. Soc. 106, 6395-6401.

La Mar, G.N., Pande, U., Hauksson, J.B., Pandey, R.K., and Smith, K.M. (1989) J. Am. Chem. Soc. 111, 485-491.

La Mar, G.N., Hauksson, J.B., Dugad, L.B., Liddell, P.A., Venkataramana, N., and Smith, K.M. (1991) J. Am. Chem. Soc. 113, 1544-1550.

Landrigan,, P.J. (1989) Br. J. Ind. Med. 46, 593-596.

Lane, R.S., Rangeley, D.M., Liem, H.H. (1973) Br. J. Haematol. 25, 533-540

Lecomte, J.T.J., and La Mar, G.N. (1985) Biochemistry 24, 7388-7395.

Lecomte, J.T.J., and Cocco, M.J. (1990) Biochemistry 29, 11057-11067.

Mabbutt, B.C., and Wright, P.E. (1985) Biochim. Biophys. Acta 832, 175-185.

Mahaffy, K.R. (1985) Dietary and Environmental Lead: Human Health Effects, Elsevier Scientific, New York.

Marion, D., and Wüthrich, K. (1983) Biochem.Biophys. Res. Commun. 113, 967-974.

McGrath, T.M., and La Mar, G.N. (1978) Biochim. Biophys. Acta 534, 99-111.

McLachlan, S.J., La Mar, G.N., and Lee, K.-B. (1988) Biochim. Biophys. Acta 957, 430-445.

Moore, C.D., and Lecomte, J.T.J. (1990) Biochemistry 29, 1984-1989.

Morgan, W.T., and Muller-Eberhard, U. (1976) Arch. Biochem. Biophys. 176, 431-441.

Morgan, W.T., Sutor, R.P., and Muller-Eberhard, U. (1976) Biochim. Biophys. Acta 434, 311-323.

Morgan, W.T., and Vickery, L.E. (1978) J. Biol. Chem. 253, 2940-2945.

Morgan, W.T., Muster, P., Tatum, F.M., McConnell, J., Conway, T.P., Hensley, P., and Smith, A. (1988) J. Biol. Chem. 263, 8220-8225.

Morgan, W.T., and Smith, A. (1984) J. Biol. Chem. 259, 12001-12006. 
Morgan, W.T., Muster, P., Tatum, F., Kao, S., Alam, J., and Smith, A. (1993) J. Biol. Chem. 268, 6256-6262.

Morgan, D.P. (1982) Recognition and Management of Pesticide Poisonings. 3rd ed. EPA-540/9-80-005, U.S. Environmental Protection Agency.

Muller-Eberhard, U.,and Grizzuti, K. (1971) Biochemistry 10, 2062-2066.

Muller-Eberhard, U. (1988) Methods Enzymol. 163, 536-565.

Muller-Eberhard, U. and Nikkilä, H. (1989) Semin. Hematol. 26, 86-104.

Muster, P., Tatum, F.M., Smith, A., and Morgan, W.T. (1988) FASEB J. 2, A746 (Abstract).

Muster, P., Tatum, F., Smith, A., and Morgan, W.T. (1991) J. Protein. Chem. 10, 123-128.

Nikkilä, H., Gitlim, J.D., and Muller-Eberhard, U. (1991) Biochemistry 30, 823-829.

iNoiva, R., Pete, M.J., and Babin, D.R. (1987) Comp. Biochem. Physiol. 88B, 341-347.

Pastemack, R.F., Francesconi, L., Raff, D., and Spiro, E. (1973) Inorg. Chem. 12, $2606-2611$.

Peyton, D.H. (1991) Biochem. Biophys. Res. Commun. 175, 515-519.

Rajarathnam, K., La Mar, G.N., Chiu, M.L., and Sligar, S.G. (1992) J. Am. Chem. Soc. 114, 9048-9058.

Rance, M., Sørensen, O.W., Bodenhausen, G., Wagner, G., Ernst, R.R., and Wüthrich, K. (1983) Biochem. Biophys. Res. Commun. 117, 479-485.

Rawn, J.D. (1989) Biochemistry, Neil Patterson Publishers, North Carolina.

Sandstrom, J. (1982) Dynamic NMR Spectroscopy, Academic Press, New York.

Satterlee, J.D. (1986) Annua. Rep. NMR Spectrosc. 17, 79-178.

Satterlee, J.D., Erman, J.E., Mauro, J.M., and Kraut, J. (1990) Biochemistry 29, 8797-8804.

Scheer, H., and Katz, J.J. (1975) in Porphyrins and Metalloporphyrins (Smith, K.M., Ed.) pp 399-524, Elsevier, Amsterdam.

Schweitzer-Stenner, R., Dannemann, U., and Dreybrodt, W. (1992) Biochemistry 31, 694-702.

Seiler, H.G., and Sigel, N. (1988) "Handbook on Toxicity of Inorganic Compounds" Marcel Dekker, New York, 297-300.

Shaka, A.J., Keeler, J., and Freeman, R. (1983) J. Magn. Reson. 53, 313-340. 
Sinclair, P.R., Bement, W.J., Gorman, N., Liem, H.H., Wolkoff, A.W. and Muller-Eberhard, U. (1988) Biochem. J. 256, 159-165.

Solar, I., Muller-Eberhard, U., and Shaklai, N. (1989) FEBS Lett. 256, 225-229.

Smith, A., and Morgan, W.T. (1981) J. Biol. Chem. 256, 10902-10909.

Smith, A., Tatum, F.M., Muster, P., Burch, M.K., and Morgan, W.T. (1988) J. Biol. Chem. 263, 5224-5229.

Smith, A., and Hunt, R.C. (1990) Eur. J. Cell Biol. 53, 234-245.

Spencer, H.T., Pete, M.J., and Babin, D.R. (1990) Int. J. Biochem. 22, 367-377

Stong, J.D., and Hartzell, C.R. (1976) Bioinorg. Chem. 5, 219-233.

Takahashi, N., Takahashi, Y., and Putnam, F.W. (1985) Proc. Nat. Acad. Sci., U.S.A. 82, 73-77.

Takano, T. (1977a) J. Mol Biol. 110, 537-568.

Takano, T. (1977b) J. Mol. Biol. 110, 569-584.

Tappel, A.L. (1955) J. Biol. Chem. 217, 721-733.

Teale, F.W.J. (1959) Biochim. Biophys. Acta 35, 543.

Thanabal, V., de Ropp, J.S., and La Mar, G.N. (1987) J. Am. Chem. Soc. 109, 7516-7525.

Traylor, T.G., and Berzinis, A.P. (1980) J. Am. Chem. Soc. 102, 2844 - 2846.

Tsutsui, K., and Mueller, G.C. (1982) Anal. Biochem. 121, 244-250.

Vincent, S.H., Grady, R.W., Shaklai, N., Snider, J.M., and Muller-Eberhard, U. (1988) Arch. Biochem. Biophys. 265, 539-550.

Wochner, R.D., Spilberg, I., Iio, A. (1974) N. Engl. J. Med. 290, 822-826.

Wüthrich, K. (1986) NMR of Proteins and Nucleic Acids, pp. 28 - 31, Wiley-Interscience, New York.

Yee, S., and Peyton, D.H. (1991) FEBS Lett. 290, 119-122.

Yu, L.P., La Mar, G.N., and Mizukami, H. (1990) Biochemistry 29, 2578-2585.

Yu, L.P., La Mar, G.N., and Rajarathnam, K. (1990) J. Am. Chem. Soc. 112, 9527-9534. 